\title{
Developing New Nanoprobes from Semiconductor Nanocrystals
}

\author{
by
}

Aihua Fu

B.S. (Beijing University of Chemical Technology) 1998

M.S. (Rutgers, the State University of New Jersey) 2001

A dissertation submitted in partial satisfaction of the

Requirements for the degree of

Doctor of Philosophy

in

Chemistry

in the

GRADUATE DIVISION

of the

UNIVERISTY OF CALIFORNIA, BERKELEY

\author{
Committee in charge: \\ Professor A. Paul Alivisatos, Chair \\ Professor Gabor A. Somorjai \\ Professor Alexander Katz
}

Spring, 2006 
The dissertation of Aihua Fu is approved:

Chair $\ldots$ Date
$\ldots$ Date $\longrightarrow$ Date

University of California, Berkeley

Spring, 2006 
Developing New Nanoprobes from Semiconductor Nanocrystals

Copyright 2006

by

Aihua Fu 


\begin{abstract}
Developing New Nanoprobes from Semiconductor Nanocrystals
\end{abstract}

Aihua Fu

Doctor of Philosophy in Chemistry

University of California, Berkeley

Prof. A. Paul Alivisatos, Chair

In recent years, semiconductor nanocrystal quantum dots have garnered the spotlight as an important new class of biological labeling tool. With optical properties superior to conventional organic fluorophores from many aspects, such as high photostability and multiplexing capability, quantum dots have been applied in a variety of advanced imaging applications. This dissertation research goes along with large amount of research efforts in this field, while focusing on the design and development of new nanoprobes from semiconductor nanocrystals that are aimed for useful imaging or sensing applications not possible with quantum dots alone. Specifically speaking, two strategies have been applied. In one, we have taken advantage of the increasing capability of manipulating the shape of semiconductor nanocrystals by developing semiconductor quantum rods as fluorescent biological labels. In the other, we have assembled quantum dots and gold nanocrystals into discrete nanostructures using DNA. The background information and synthesis, surface manipulation, property characterization and applications of these new nanoprobes in a few biological experiments are detailed in the dissertation.

Professor A. Paul Alivisatos

Dissertation Committee Chair 
To my parents, Qiaoyun and Shuai, my husband, Xiangdong, and my son, Anthony, who give me inspiration, courage, and happiness in life. 


\section{Contents}

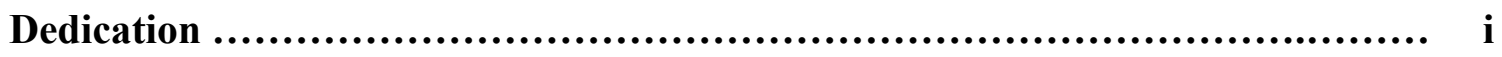

Contents .......................................................................... ii

List of figures............................................................... iv

List of tables ............................................................... viii

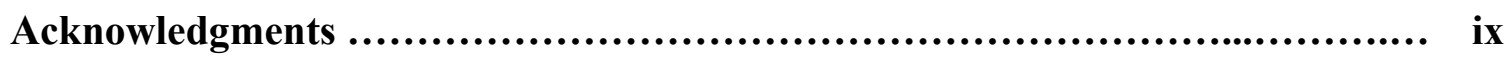

1 Introduction: Semiconductor Nanocrystals and Their Applications in Biological

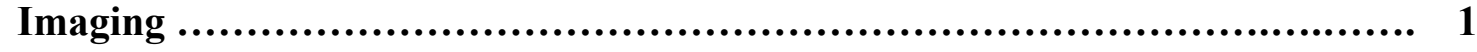

1.1 Semiconductor Nanocrystals for Biological Imaging $\ldots \ldots \ldots \ldots \ldots \ldots \ldots \ldots \ldots . \quad 1$

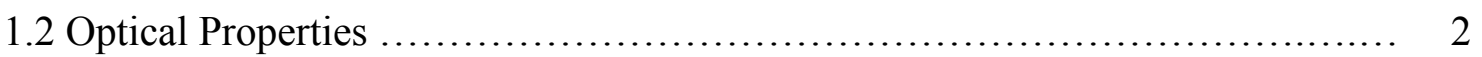

1.3 Semiconductor Nanocrystals for In Vitro Imaging .......................... 4

1.4 Semiconductor Nanocrystals for In Vivo Imaging ........................ 7

1.5 Semiconductor Nanocrystals for Neuronbiology $\ldots \ldots \ldots \ldots \ldots \ldots \ldots \ldots \ldots \ldots \ldots \ldots$

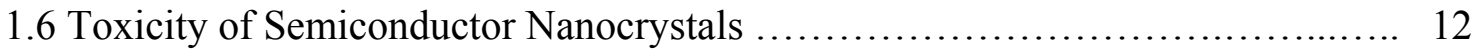

1.7 Developing New Nanoprobes from Semiconductor Nanocrystals .............. 13

2 Surface Modification of Nanocrystals for Biomedical Applications ............... 20

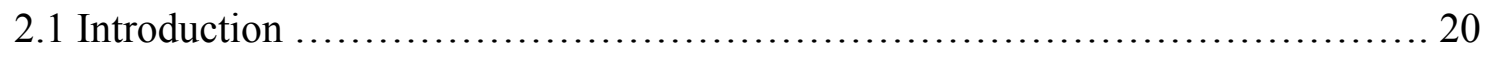

2.2 Surface Silanization of Semiconductor Quantum Rods CdSe/CdS/ZnS .......... 21

2.3 Surface Silanization of Semiconductor Quantum Dots CdSe/ZnS ............... 24

2.4 Characterization of Silanization Coating Thickness ........................ 25

2.5 Silanization of Metal Alloy and Metal Oxide Nanocrystals .................... 30

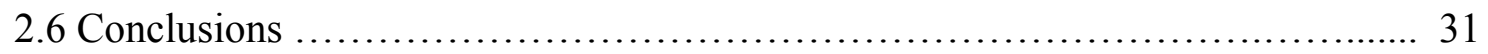


3.1 Introduction .......................................................... 34

3.2 Surface Silanization of Quantum Rods ................................ 36

3.3 Silanized Quantum Rods are Biocompatible ............................ 38

3.4 Reduced Cd2+ Leakage of Silanized Quantum Rods Compared to QDs ...... 40

3.5 Quantum Rods for Immunofluorescence Labeling ........................ 42

3.6 Quantum Rods for Single Molecule Imaging ........................... 44

3.7 Quantum Rods as Single Molecule Orientation Probes ...................... 48

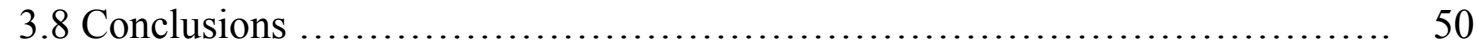

3.9 Methods .............................................................. 51

4 Discrete Nanostructures of Quantum Dots /Au with DNA .................... 61

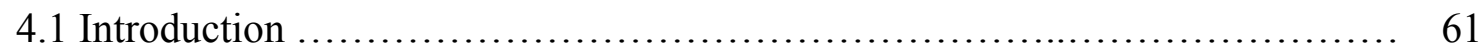

4.2 Synthesis of Discrete Nanostructures of QDs/Au with DNA ................. 63

4.3 TEM Analysis of the Nanostructure Populations ............................. 65

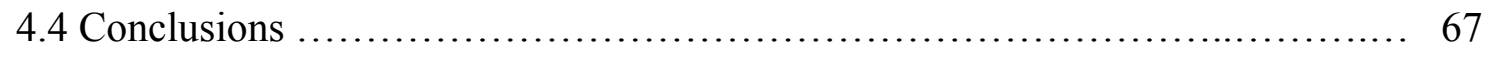

5 QDs/Au -DNA Nanostructures in Probing Activity of Hydrolytic Enzymes...... 70

5.1 Introduction ............................................................ 70

5.2 Optical Properties of Ensemble QDs/Au -DNA Nanostructures ................ 72

5.3 QDs/Au-DNA Nanostructures Compatible with Hydrolytic Enzyme Function ... 74

5.4 Comparison of Photoluminescence Effects between Experimental Results and Analytical Calculation for QDAu1 Structures .................................. 77

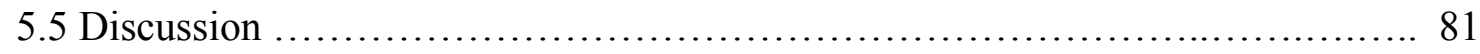

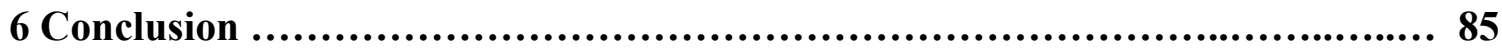




\section{List of Figures}

1.1 (a) Excitation (dashed) and fluorescence (solid) spectra of fluorescein (A) and a typical water-soluble QD sample (B) in PBS. The nanocrystals have a much narrower emission (32 $\mathrm{nm}$ compared with $45 \mathrm{~nm}$ at half maximum and $67 \mathrm{~nm}$ compared with $100 \mathrm{~nm}$ at $10 \%$ maximum), no red tail, and a broad, continuous excitation spectrum. (b) A, Size- and material-dependent emission spectra of several surfactant-coated QDs in a variety of sizes. The blue series represents different sizes of CdSe QDs with diameters of 2.1, 2.4, 3.1, 3.6, and $4.6 \mathrm{~nm}$ (from right to left). The green series is of InP QDs with diameters of 3.0, 3.5, and 4.6 nm. The red series is of InAs QDs with diameters of $2.8,3.6,4.6$, and $6.0 \mathrm{~nm}$. B, A true-color image of a series of silicacoated core $(\mathrm{CdSe})$-shell $(\mathrm{ZnS}$ or $\mathrm{CdS})$ nanocrystal probes in aqueous buffer all illuminated simultaneously with a handheld ultraviolet lamp. Reproduced with permission from [4]. 3

1.2 Defocused microscopy images of QD coupled glycine receptor in the membrane of a Hele cell. The contour intensities (dotted lines) can be fitted (solid lines) to determine the orientation $(\Theta, \Phi)$ of each QD. Reproduced with permission from [30].

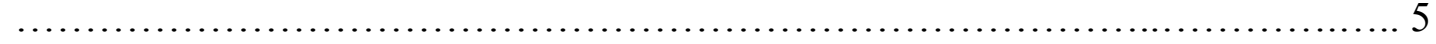

1.3 QDs were used to study mixed cell interactions in a 3-D Matrigel culture system. (A) Human mammalian epithelial MCF 10A cells (labeled with green emitting silica coated QDs) form acini structures after growing in growth-factor reduced matrigel for 10 days. (B) After the acini were formed, human breast tumor MDA-MB-231 cells (labeled with red-emitting silica coated QDs) were added to the culture. After 14-16 $\mathrm{h}$ of incubation, the tumor cells had attached to the acini. (C) The contact was fatal to the tumor cells, which were found dead surrounding the MCF 10A acinus. Most of the tumor cells had lysed, leaving transparent ghosts loosely attached to the acinus, but a few newly attached cells still retained red-emitting QDs. (D) The MCF-10A acini and all invading tumor cells; it is a superimposition of all sections, displaying the sharp edge of each cell followed by a projection of color-coded depth information so that red is the uninvolved lower portion of the MCF-10A acini and the tumor cells

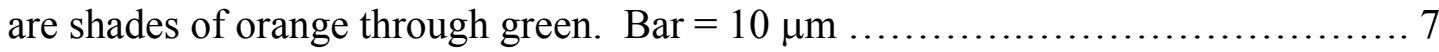

1.4 Near infrared (NIR) QD sentinel lymph node mapping in the mouse. The mouse was injected intradermally with 10pmol of NIR QDs in the left paw. Left, pre-injection NIR autofluorescence image; middle, 5 min post injection white light color video images; right, 5 min post-injection NIR fluorescence image. An arrow indicates the putative axillary sentinel lymph node. Reproduced with permission from [11] .......9 
1.5 QDs as marker for glycine receptor (GlyR) localization in neurons. QD labeled GlyR (red) was detected over the somatodendritic compartment identified by microtubuleassociated protein-2 (green). Arrows mark clusters of QD labeled GlyRs located on dendrites. Reproduced with permission from [10] .... 10

2.1 A cartoon to illustrate the surface silanization of nanoparticles. Cross linked silane molecules are used as priming agents, and different surface functional groups can be incorporated into silanization. 22

2.2 TEM images of silanized quantum rods (a) and QDs (b). Scale bars are 100nm. ... 24

2.3 TEM of silanized quantum rods with thicker silica shell by addition of sodium silicate

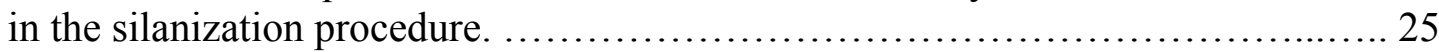

2.4 TEM images of silanized quantum rods on Ted Pella 01890-F TEM grids. Scale bars

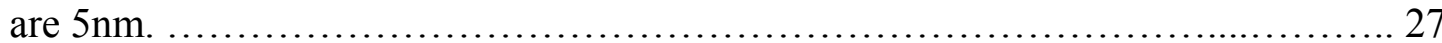

2.5 TEM of silanized quantum rods on Ted Pella 01824-F TEM grids. Scale bar is

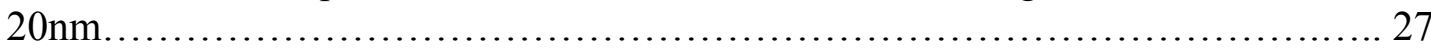

2.6 TEM of silanized quantum rods on Electron Microscopy Sciences LC225-Cu grids. Scale bars are $5 \mathrm{~nm}$. 27

2.7 Sizes of CdSe/ZnS QDs in chloroform (red) and silanized CdSe/ZnS QDs (blue) in PB buffer measured using Malvern Instruments ZetaSizer NanoZS. 29

2.8 TEM images of silanized $\mathrm{FePt}(\mathrm{a}), \mathrm{Fe} 2 \mathrm{O} 3$ with mercapto rich surfaces (b) and $\mathrm{Fe} 2 \mathrm{O} 3$ with amino rich surfaces (c). Scale bars are $20 \mathrm{~nm}$ for a and c, and $100 \mathrm{~nm}$ for $b$.

2.9 Pictures of different samples after silanization in neutral PB buffer. a, CdSe/CdS/ZnS rods; b, CdSe/ZnS dots ; c, FePt; d, Hollow $\mathrm{Fe}_{2} \mathrm{O}_{3}$ with amino rich surface; e, Hollow $\mathrm{Fe}_{2} \mathrm{O}_{3}$ with mercapto rich surface. 31

3.1 Synthesis and property characterization of Silanized Quantum Rods. (a) A cartoon illustrating silanization of quantum rods. Crosslinked silanes are priming molecules for the surface coating. (b) TEM image of silanized rods in neutral phosphate buffer. Scale bar $=100 \mathrm{~nm}$. (c) The UV-Vis absorption and emission spectra of silanized rods. The blue curve is the absorption spectrum, while the red curve is the emission spectrum. . .38

3.2 Human breast cancer cell MDA-MB-231 and colon cancer cells SW 480 cultured on top of quantum rod layer left fluorescence free area after 24 hours incubation. The size of the fluorescence free area is related to the invasiveness of the cancer cell, as being reported previously while cells were cultured on quantum dot layer. .......... 39

3.3 Silanized rods are biocompatible and not toxic to living cells. The red fluorescence in the images is from quantum rods in human breasts cancer cells MDA-MB-231 after $1 \mathrm{~h}$ (left) and $24 \mathrm{~h}$ (right) transfected with Chariot ${ }^{\mathrm{TM}}$. These are merged images of transmission and fluorescent micrograms. Scale bar is $20 \mu \mathrm{m}$. 40 
3.4 Rod shaped nanocrystals reduce the $\mathrm{Cd}^{2+}$ leakage significantly over that of spherical nanocrystals. The $\mathrm{Cd}^{2+}$ leakage was assayed by ICP/OES. 41

3.5 TEM images of quantum rods (a) and QDs (b) used in the $\mathrm{Cd} 2+$ leakage comparation. Scale bars are $100 \mathrm{~nm}$. 42

3.6 (a) The scheme for antibody bioconjugation of quantum rods with surface mercapto functional groups. (b) Electrophoresis analyses of quantum rods/dots bioconjugation. Top, quantum rods/dots conjugated with $\mathrm{F}\left(\mathrm{ab}^{\prime}\right)_{2}$ fragment of goat anti-mouse IgG antibody. Bottom, quantum rods/dots conjugated with whole goat anti-mouse IgG antibody. The conjugates moved slower than the free nanocrystals (control) due to the linkage with antibodies. 43

3.7 Immunofluorescence labeling of breast cancer cell marker Her2 on breast cancer cells SK-BR-3. The Her2 marker was labeled with mouse anti-Her2 antibody and goat anti-mouse $\operatorname{IgG~} \mathrm{F}\left(\mathrm{ab}^{\prime}\right)_{2}$ conjugated quantum rods/dots. The bottom images show that there is minimum binding of free nanocrystals to the anti-Her2 antibody treated cells.

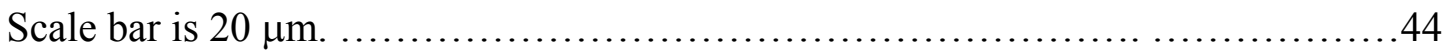

3.8 Fluorescence microscope images show that at single molecule level, quantum rods (a) are much brighter than quantum dots (b). (c) Statistical results of rods (top) and dots (bottom) from 15 image sequences. The color plots are the Ln of number of particles at different threshold intensity and threshold image number (the number of images that one particle at least appears in the 15 image sequence, for example, using a threshold image number 5 selects all the particle appears in at least 5 images of the 15 image sequence.) The $\mathrm{Ln}$ (Number of Particles) plots clearly illustrate how hard it is to different particles from background noise for QDs, as one can see that the particle number decreased all the way down dramatically when the threshold and threshold image number are increased. However, for the rod case, there is a broad region where the number of rod particles is relatively the same with increasing threshold and threshold image number. The inserts are the histograms of particle intensity distribution when using threshold 10 , and threshold image number 5 , which pick up 1624 rods giving a mean intensity of 29.5 , and pick up 883 dots with a mean intensity of 12.7. Please note the much smaller number of particles picked up for QDs. In another word, only bright dot particles with relatively long on time of dot are considered in the statistics. 47

3.9 Single rods (indicated by arrows) are still very bright inside live MDA-MB-231

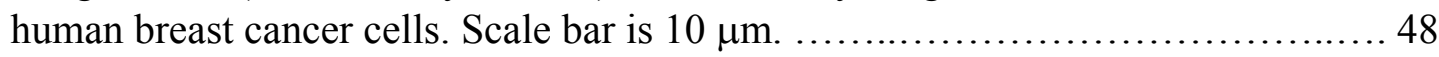

3.10 Polarized emission from single silanized quantum rods. 49

4.1 Gel-electrophoresis migration patterns of $\mathrm{QD} / \mathrm{Au}$ nanostructures. The same gel is illuminated under UV (to see QDs by fluorescence, left panel) and under white light (to represent $\mathrm{Au}$ through absorption, right panel). Discrete bands correspond to different number of $\mathrm{Au}$ (illustrated by cartoon) bound to the QDs through DNA

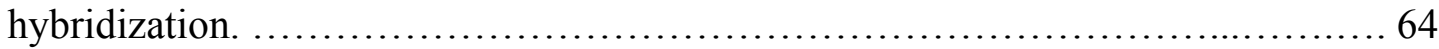


4.2 Electrophoresis mobility of different samples. The top panel shows QDs. The bottom panel shows Au. Discrete bands only appear when QDs and Au have complimentary DNA on them, which rules out nonspecific binding among QDs, Au and DNA.

4.3 TEM images of discrete nanostructures of QDs/Au extracted from corresponding bands after gel-electrophoresis. (a) $\mathrm{QD}(\mathrm{Au})_{1}$. (b) $\mathrm{QD}(\mathrm{Au})_{2}$. (c) $\mathrm{QD}(\mathrm{Au})_{3}$. (d) $\mathrm{QD}(\mathrm{Au})_{4}$. The scale bar is $100 \mathrm{~nm}$. (e) Structure populations and pair distribution functions of $\mathrm{QD}(\mathrm{Au})_{2}$ with 50 mer DNA (top), and 100mer DNA connected $\mathrm{QD}(\mathrm{Au})_{2}$ (middle) and $\mathrm{QD}(\mathrm{Au})_{3}$ structures (bottom) based on quantitative analysis of TEM images of corresponding samples.

5.1 (A) Gel-electrophoresis separation of 50mer DNA connected QDAu nanostructures, the left image showing the fluorescence from QD under UV light, and the right image showing the absorption of white light by Au. (B) The photoluminescence of samples extracted from each nanostructures bands are compared with free QD at the same concentration determined by ICP measurements. PL quenching is observed in each sample. 74

5.2 (A) A cartoon illustrating the cleavage of the linker biomolecules by hydrolytic enzyme. (B) Gel electrophoresis of the digestion of EcoRI on $\lambda$ DNA. The left lane shows the digestion in the dialysis buffer of $50 \mathrm{mM} \mathrm{NaCl}, 10 \mathrm{mM} \mathrm{MgCl}$ and $0.5 \mathrm{X}$ TBE; the middle lane shows the digestion in the original EcoRI buffer; the right lane shows the movement of DNA ladder in agarose gel. (C) The effect of Au upon the PL of QD used in probing hydrolytic enzyme function. For example, in 50mer DNA connected nanostructure sample extracted from the second structure gel band, the PL of QD is quenched; by choosing double strand DNA containing EcoRI recognition sites, a PL increase can be observed as EcoRI are cleaving the DNA and breaking the

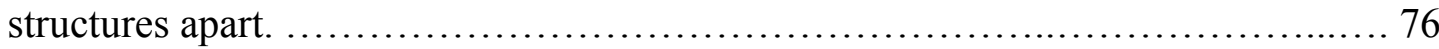

5.3 Experimental (unconnected points with error bars) and theoretical (colored lines) results of the effect of Au upon the PL of QD for 50mer, 65mer, 80mer and 100mer DNA connected QDAu 1 Nanostructures. 79

6.1 New nanoprobes developed in this dissertation research. (a) The TEM images of QDAu nanostructures with different number of Au around the central QD. (b) The TEM images of silanized quantum rods. 85 


\section{List of Tables}

5.1 The Au effect upon the PL of QD for each structure type is obtained by solving a matrix built on the assumption that the PL of a sample is equal to the sum of the PL of each structure type in the sample. The standard deviation is obtained through error analysis based on experimental measurements of uncertainty of each component in

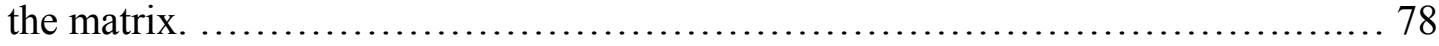




\section{Acknowledgements}

I have been fortunate to spend the past 5 years at Berkeley and work on cutting-edge projects. The work presented here would have not been accomplished without the generous help from many talented people.

First and foremost, I would like to thank my advisor, professor Paul Alivisatos, for a lot of guidance and discussions. His encouragement during pitfalls of projects and his constructive criticism when projects at completion are invaluable in my learning experience for scientific research.

I am grateful to all the people in the Alivisatos group for building such an enjoyable environment to learn, create and grow. During my early times here, Daniele, Wolfgang, Shara, Christine, Liangshi and Liberato helped me a lot in starting the experiments. Throughout these years, I am fortunate to have worked together with Deborah, Emory, Haitao, Yadong, Yi, Teresa, Carsten, Bryce, Stevo, Alex, Josh, Shelley, Sassan, Andreu, Jennifer, Jonason, Kristie, Kimani, Can and other group memebers, all of these highly talented and friendly people have added sweet flavor to my scientific growing "pains". Related to what presented in this dissertation work, I would like to thank Ben for taking the single rod polarization spectra, thank Christine for analyzing the TEM data of QDAu nanostructures in chapter 4, thank Kristie for programing and analyzing the single rod and dot fluorescent imaging data, thank Daniela for taking TEM of rod with thick 
silanization coating, thank for the access to Frechet group ZetaSizer NanoZS for dynamic light scattering measurement and to Bustamante group gel scanner, thank Yadong for giving me $\mathrm{FePt}$ and Andreu for giving me $\mathrm{Fe}_{2} \mathrm{O}_{3}$ particles for silanization, and thank Kimani for being convinced and applying silaniztion as surface modification for rod alignment. I also want to thank Rita and James for taking good care of many important things in the lab that allow us to focus more on research.

Outside of the Alivisatos group, I would like to thank Prof. Carolyn Larabell and Dr. Weiwei Gu from physical bioscience division of LBL for being very helpful in rod biological labeling experiments. I would like to thank Dr. Frank Chen from life science division of LBL for good advice and discussion for bioapplications of QDAu nanostructures. I also want to thank Prof. Haw Yang and some members in his group, including Hauyee, Kai and Lucas for helpful discussions and being generous in instruments usage at their lab. Kai did the calculation of PL effect in $\mathrm{QDAu}_{1}$ nanostructures shown in chapter 5 .

There are too many people to count for as being vital in the success of research projects described here, such as Paul Brooks in the natural resource center for training me for the ICP measurement, and people from the glass and machine shops. I may forget some important people at this point, but I am very grateful to you and please forgive me for not mentioning your name here.

Finally, I would like to thank my parents, Qiaoyun and Shuai, thank my brother and Sisters, Aiwu, Aijun, Aimin and Aiwen, for their constant support and love and care. I would like to thank my husband, Xiangdong, for his unconditional love and faith in me. I 
am also very grateful that my baby, Anthony (Xiaoshan) has been such a good boy and brought me a different set of experimenting and fun.

Financial supports for the dissertation research include grants from DARPA, DOE and NIH. 


\section{Chapter 1}

\section{Introduction: Semiconductor Nanocrystals and Their Applications in Biological Imaging}

\subsection{Semiconductor Nanocrystals for Biological Imaging}

Semiconductor nanocrystals, also called quantum dots (QDs), are a new class of fluorescent biological labels. Originating from quantum confinement of electrons and holes within the nanocrystal core material, the fluorescence from QDs is unique compared to that from traditional organic fluorophores. For example, QDs exhibit high photo stability, broad absorption and narrow and symmetric emission spectra, slow excited state decay rate and large absorption cross section ${ }^{1}$. Their emission color can be continuously tuned from ultraviolet to visible and infrared wavelengths by changing the size and chemical composition of the semiconductor core nanocrystal. Growing a semiconductor shell with a larger band gap improves the quantum confinement resulting in very bright and highly stable, chemically as well as optically, semiconductor fluorophores ${ }^{2-3}$. QDs offer an exciting potential to overcome many of the limitations encountered by traditional organic dyes and genetically engineered fluorescent proteins. Since their introduction into biological imaging in $1998^{4-5}$, an enormous body of research 
has emerged focusing on the synthesis, photophysical property characterization and bioconjugation ${ }^{6-9}$ of QDs. Advanced molecular and cellular imaging with QDs has also been realized ${ }^{10-11}$.

Biocompatible QDs find utility not only as a basic bio-labeling tool, but also as a key building block for complex multi-functional bio-probes. Their large surface area may be tailored to bind both target selective molecules and therapeutic molecules, enabling spontaneous delivery of treatments to a probed disease area. Complex nanostructures formed by linking QDs and gold nanoparticles through DNA hybridization or streptavidin-biotin interaction have also been realized ${ }^{12-14}$ and applied in sensing biomolecular concentration ${ }^{14}$. Although QDs have been utilized in a broad range of imaging applications to date, their versatility for advanced biomedical applications remains to be fully explored.

\subsection{Optical Properties}

The size dependent optical properties of QDs result from their quantum-confined electronic states ${ }^{15}$. Just as in the "particle in a box" model, excitons in smaller nanocrystals experience stronger quantum confinement, resulting in larger photoluminescence energy. Figure 1 shows the typical absorption and emission spectra of water-soluble QDs. Their emission wavelength can be continuously tuned from $400 \mathrm{~nm}$ to $2000 \mathrm{~nm}$ by changing both the nanocrystal size and composition ${ }^{16}$.

In contrast to conventional fluorophores, QDs have broad absorption and narrow and symmetric emission spectra. These features allow concurrent imaging of multiple 
entities in a single biological experiment, a quite difficult task with standard fluorophores since their relatively narrow excitation and broad emission spectra often result in spectra overlap ${ }^{2}$. Another advantage of QDs is that they are highly resistant to metabolic degradation and are hundreds of times more photo stable than conventional fluorophores. In addition, QDs often have a large Stokes shift, that is, a large separation between the excitation wavelength and the emission maxima, this has the effect of reducing autofluorescence, resulting in a several fold increase in sensitivity versus organic fluorophores ${ }^{17}$.

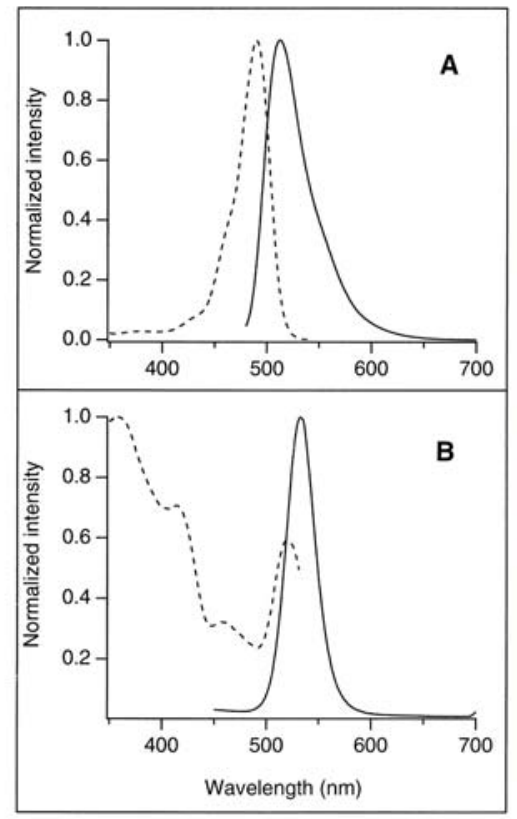

(a)

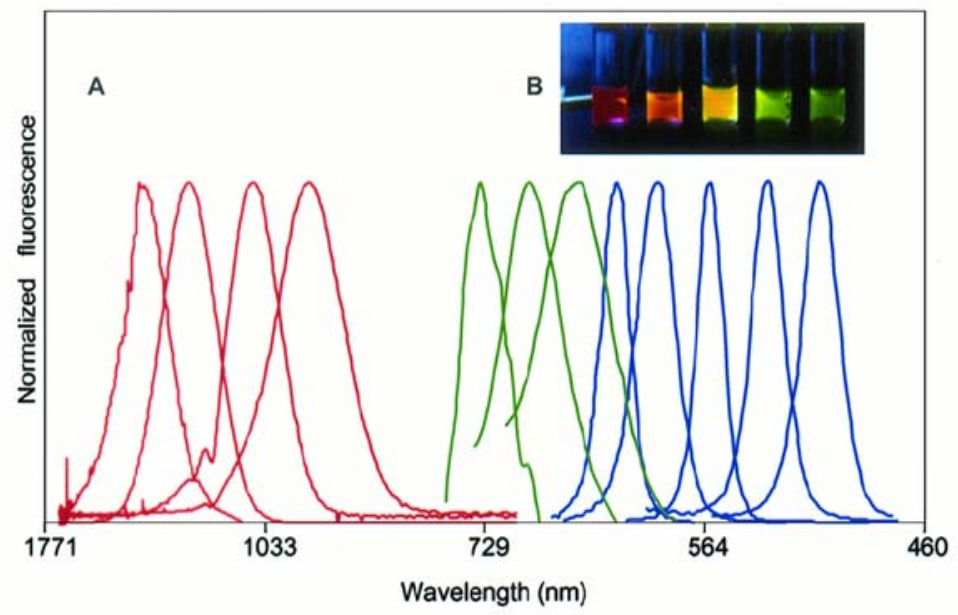

(b)

Figure 1.1 (a) Excitation (dashed) and fluorescence (solid) spectra of fluorescein (A) and a typical water-soluble QD sample (B) in PBS. The nanocrystals have a much narrower emission (32 nm compared with $45 \mathrm{~nm}$ at half maximum and $67 \mathrm{~nm}$ compared with $100 \mathrm{~nm}$ at 10\% maximum), no red tail, and a broad, continuous excitation spectrum. (b) A, Size- and material-dependent emission spectra of several surfactant-coated QDs in a variety of sizes. The blue series represents different sizes of CdSe QDs with diameters of 2.1, 2.4, 3.1, 3.6, and $4.6 \mathrm{~nm}$ (from right to left). The green series is of InP QDs with diameters of 3.0, 3.5, and $4.6 \mathrm{~nm}$. The red series is of InAs QDs with diameters of 2.8, 3.6, 4.6, and $6.0 \mathrm{~nm}$. B, A true-color image of a series of silica-coated core (CdSe)-shell ( $\mathrm{ZnS}$ or $\mathrm{CdS}$ ) nanocrystal probes in aqueous buffer all illuminated simultaneously with a handheld ultraviolet lamp. Reproduced with permission from [4]. 
For QDs, quantum yields can be as high as 0.89 at room temperature ${ }^{18}$; molar extinction coefficients, about $10^{5}-10^{6} \mathrm{M}^{-1} \mathrm{~cm}^{-1}$, are 10 to 100 times larger than most organic dyes ${ }^{19}$, and they have orders of magnitude larger two-photon absorption cross section ${ }^{20}$. Optical properties of QDs are usually unaffected by conjugation to biomolecules. Thus they are both highly stable and bright probes, especially suitable for photon-limited in vivo studies and continuous tracking experiments over extended time periods. A more extensive discussion of photophysical properties of QDs is presented by Grecco et al. ${ }^{21}$.

\subsection{Semiconductor Nanocrystals for In Vitro Imaging}

QDs have been very successful in immunofluorescent labeling. With continuous efforts in developing high quality biocompatible QDs, nanoparticles conjugated to antibodies, peptides and DNA have been prepared and targeted to cells and tissues specifically, allowing multiplexed labeling and long term studies that can not be achieved by using standard dyes ${ }^{22-27}$. Although QDs and organic dyes can have comparable quantum yields, the larger absorption cross-section of the nanocrystal results in a much stronger photoluminescence signal. The sustained strong signal from a single nanoparticle was used to track dynamic cellular processes over time scales unavailable using organic fluorophores ${ }^{10}$. Recently, Dahan et al. developed a method to study single

nanocrystal fluorescence patterns using defocused microscopy ${ }^{28}$. By relating these patterns to the structures of the nanocrystal emission dipoles they were able to determine the three-dimensional orientation of the nanoparticles, and successfully applied this technique to track the orientation of single membrane receptor in live cells (Figure 2). 
With continuous efforts in elucidating the photophysics of single QDs, there will be increasing interest in their application as fluorescent emitters for studying dynamic biophysical processes. For example, Yildiz and Selvin demonstrated that total internal reflection microscopy used in conjunction with organic fluorophores can produce fluorescence imaging with one nanometer accuracy (FIONA) ${ }^{29}$. In this technique, a large number of photons are collected over time from a single dye molecule, allowing researchers to locate the center of the fluorescent pattern with high precision. This technique has been applied to unravel the walking mechanism of the molecular motors myosin V, myosin VI and kinesin. Although the presented experimental results were from an organic dye, the authors believed that using QDs would provide at least a 10-fold improvement in time resolution and are extending the applications of FIONA in motor movements with QDs.
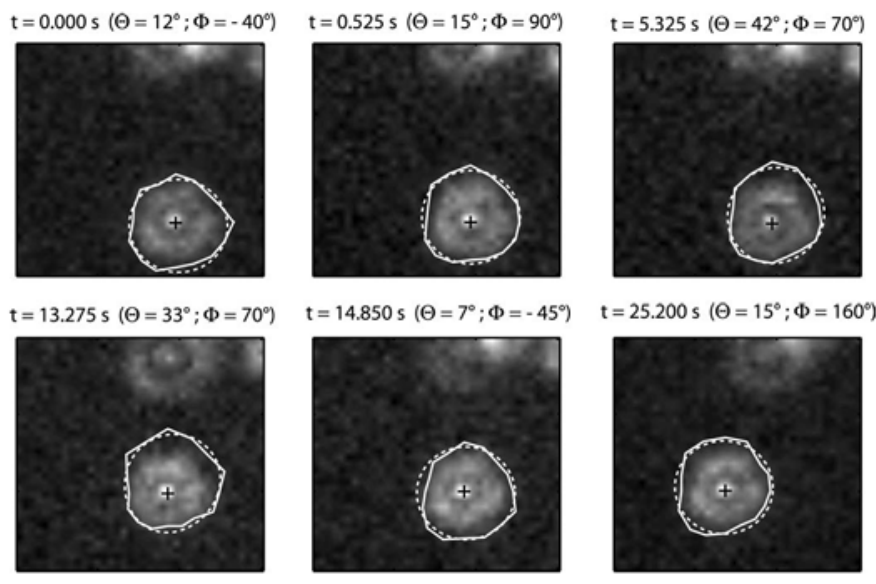

Figure 1.2. Defocused microscopy images of QD coupled glycine receptor in the membrane of a Hele cell. The contour intensities (dotted lines) can be fitted (solid lines) to determine the orientation $(\Theta, \Phi)$ of each QD. Reproduced with permission from [30].

QDs can be uptaken by live cells with no need of any functionalization, possibly due to the characteristic size range and good biocompatibility. Pellegrino et al. studied 
the phagokinetic tracks left on a homogenous layer of silanized $\mathrm{CdSe} / \mathrm{ZnS}$, and demonstrated that QDs can be used as a two-dimensional in vitro invasion assay for discriminating between non-invasive and invasive cancer cell lines ${ }^{29}$. This technique provides a new tool for quantifying tumor cell invasiveness. Internalized QDs are also powerful probes for long-term studies of cell-cell interactions. They have been used to examine the interactions of human mammary epithelial tumor cells with normal cells growing in a 3-D culture system. The tumor cell behavior around polarized normal cell clusters was clearly demonstrated when labeling tumor cells and normal cells with nanocrystals of different emission colors. The high photostability of the QDs is critical in the tracking and imaging of these cocultures for extended time periods (up to 14 days) and cannot be replaced by organic fluorophores ${ }^{30}$ (Figure 1.3).

Applying semiconductor nanoparticles for in vitro labeling allows fluorescent and electron microscopy ${ }^{10,18,31}$ imaging of the same probe, so that information on both temporal dynamics and high-resolution cellular localization can be obtained ${ }^{10}$. The fluorescence and electron density properties of QDs were also utilized by Nisman et al. to label a nuclear protein on cell sections and to correlate the fluorescence and TEM data. They also employed QDs in conjunction with immunogold to colocalize proteins at the ultrastructure level. Moreover, by obtaining cadmium elemental maps of $\mathrm{CdSe} / \mathrm{ZnS}$ distributed on a nuclear structure, the authors demonstrated the potential of using quantum dots as tags for electron spectroscopic imaging to colocalize multiple proteins 31. 

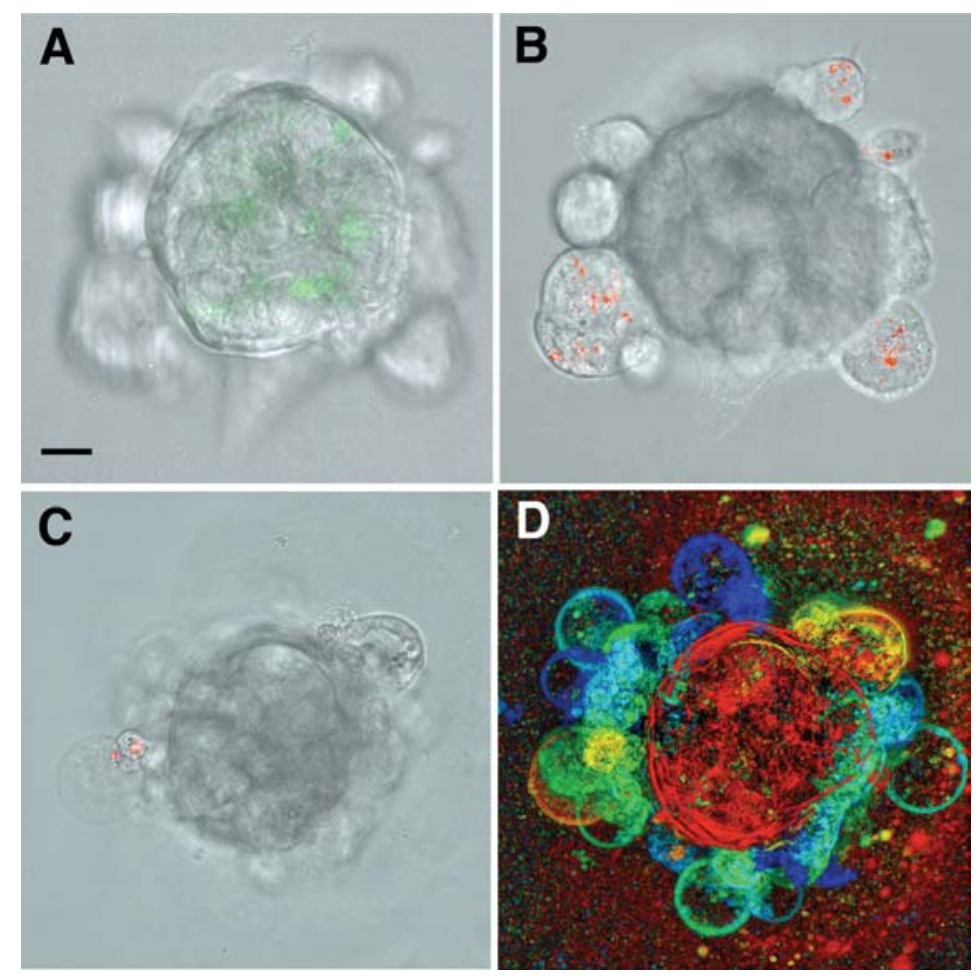

Figure 1.3. QDs were used to study mixed cell interactions in a 3-D Matrigel culture system. (A) Human mammalian epithelial MCF 10A cells (labeled with green emitting silica coated QDs) form acini structures after growing in growth-factor reduced matrigel for 10 days. (B) After the acini were formed, human breast tumor MDA-MB-231 cells (labeled with red-emitting silica coated QDs) were added to the culture. After 14-16 h of incubation, the tumor cells had attached to the acini. (C) The contact was fatal to the tumor cells, which were found dead surrounding the MCF 10A acinus. Most of the tumor cells had lysed, leaving transparent ghosts loosely attached to the acinus, but a few newly attached cells still retained red-emitting QDs. (D) The MCF-10A acini and all invading tumor cells; it is a superimposition of all sections, displaying the sharp edge of each cell followed by a projection of color-coded depth information so that red is the uninvolved lower portion of the MCF$10 \mathrm{~A}$ acini and the tumor cells are shades of orange through green. $\mathrm{Bar}=10 \mu \mathrm{m}$.

\subsection{Semiconductor Nanocrystals for In vivo Imaging}

The extreme brightness of QDs and their resistance to photobleaching enable continuous exposure under laser illumination for an extended period of time, making them especially useful for in vivo imaging. Progress in nanocrystal synthesis, coating and surface modification has significantly enhanced their applications in tracking and imaging. Efforts in optimizing the surface coating for in vivo imaging have shown that specific polyethylene glycol (PEG) coatings result in longer circulation time ${ }^{18,32}$, 
enhanced stability ${ }^{18}$, and minimal nonspecific deposition ${ }^{18,33}$, which are essential elements for in vivo imaging.

While the first in vivo targeting experiment imaged the histological sections of mouse organs after intravenous injection of peptide conjugated QDs, recent applications primarily focus on live animal imaging combined with multi-photon microscopy or with the use of near infrared nanocrystals.

The large two-photon absorption cross section of QDs allows for more efficient probing of thick specimens by multiphoton excitation microscopy ${ }^{34}$. With the use of this technique, fluorescence signals were able to be detected hundreds of microns deep through the skin of live mice ${ }^{20}$ and thick tissue specimens ${ }^{35}$. Stroh and colleagues recently explored the use of QDs in anatomical imaging with multiphoton microscopy. Unlike traditional fluorescence labeled dextran vessel markers, the nanocrystals distinctly differentiate tumor vessels from perivascular cells and matrix. This group also assessed the ability of nanocrystals to monitor tumor and cell trafficking ${ }^{36}$. These findings show the potential uses of QDs in designing drug delivery particles and tumor pathophysiological studies.

Tracking and imaging nanocrystals in live animals has been achieved by Nie's group. QDs were conjugated to the antibody specific for the prostate cancer cell marker PSMA. After injection into mice that had been transplanted with human prostate cancer cells, the quantum dot-tagged PSMA antibodies recognized and bound at the tumor site and were clearly imaged in vivo. Due to the large absorption coefficient and long lifetime, in vivo images of nanoparticles were much brighter and more sensitive than images with green fluorescent protein ${ }^{32}$. 
One challenge in live animal imaging is the significant autofluoresent background. Several strategies can be applied to solve this problem. One approach is to use spectral imaging or emission scanning microscopy to separate the nanocystal fluorescence signal from background noise ${ }^{32,35}$. Since nanocrystals have narrow emission bands, this method also allows for multicolor tracking of up to five different nanoparticles in vivo ${ }^{35}$. An even more effective solution is to move from visible light to near infrared (NIR) since most tissue chromophores absorb weakly at such long wavelengths. Another advantage of NIR imaging is deeper penetration. Kim and coworkers first demonstrated the use of NIR QDs to map sentinel lymph nodes (SLN) during surgical procedures ${ }^{11}$. The nanoparticles, after intradermal injection into the animal, entered the lymphatic system, and were followed using an intraoperative imaging system. The surgeon followed the flow of nanocrystals in real time with NIR image guidance, and quickly identified the position of the SLN in a precise and rapid surgical procedure. NIR nanocrystal imaging of blood vessels and beating heart through 1-2 mm of skin and tissues were also reported ${ }^{37}$.
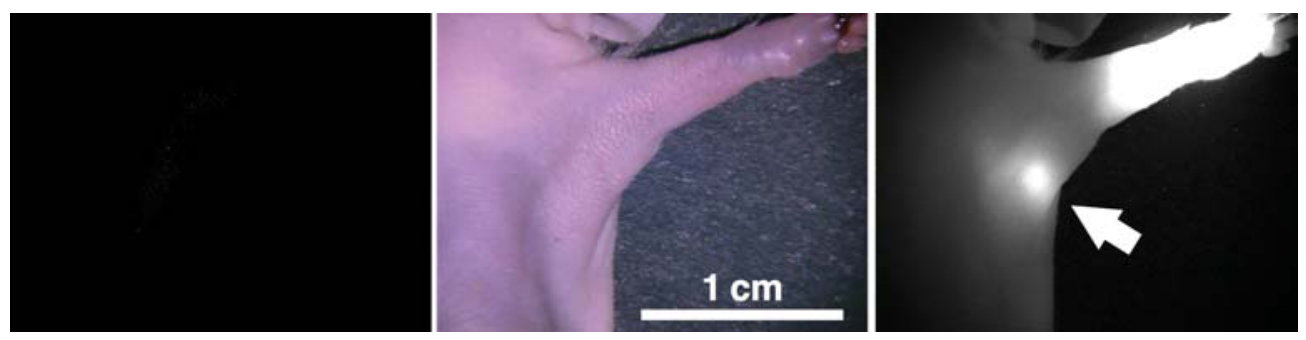

Figure 1.4. Near infrared (NIR) QD sentinel lymph node mapping in the mouse. The mouse was injected intradermally with 10pmol of NIR QDs in the left paw. Left, pre-injection NIR autofluorescence image; middle, 5 min post injection white light color video images; right, $5 \mathrm{~min}$ post-injection NIR fluorescence image. An arrow indicates the putative axillary sentinel lymph node. Reproduced with permission from [11]. 


\subsection{Semiconductor Nanocrystals for Neurobiology}

One common approach to studying neurotransporters involves the use of radiolabeled substrates or antagonists that can be monitored with high sensitivity. However, the cost and complexity involved in using radiolabelled material is high. Additionally, real time monitoring of the transporter activity is not possible. In contrast, a fluorescence-based approach allows for the localization and direct monitoring of realtime activities.

Owing to their high degree of photostability and brightness, QDs are more suitable probes than organic dyes for studies of neuronal protein or receptor dynamics

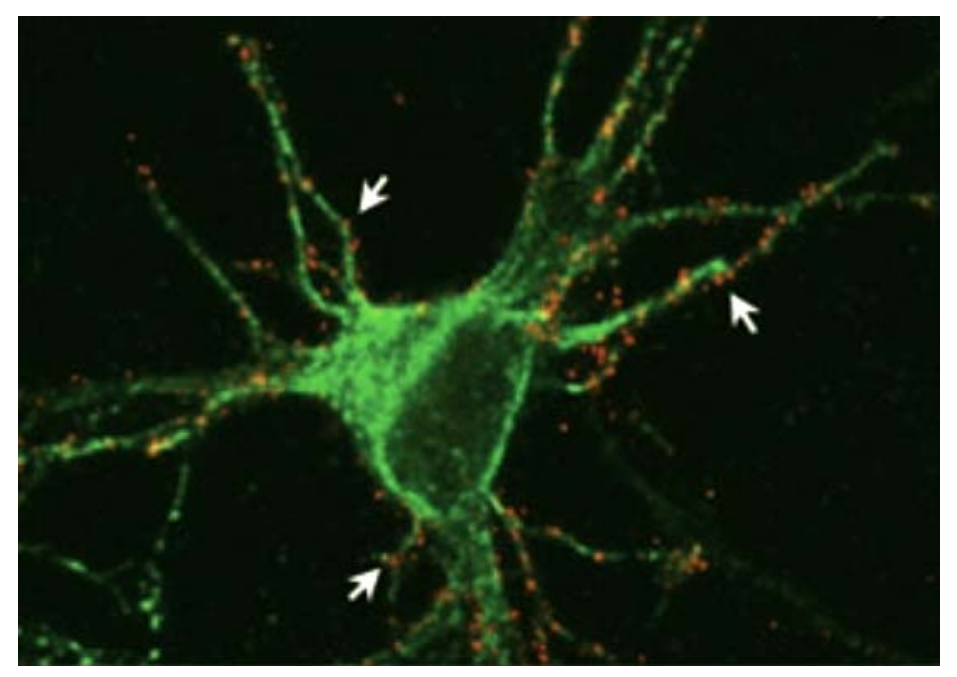

Figure 1.5. QDs as marker for glycine receptor (GlyR) localization in neurons. QD labeled GlyR (red) was detected over the somatodendritic compartment identified by microtubule-associated protein-2 (green). Arrows mark clusters of QD labeled GlyRs located on dendrites. Reproduced with permission from [10].

over an extended period of time. Semiconductor nanoparticles have been used to track individual glycine receptors, a major inhibitory neurotransmitter receptor, on the surface of cultured spinal neurons ${ }^{10}$ (Figure 1.5). Compared to Cy3 dye, fluorescent nanoparticles had significantly higher signal-to-noise ratio and allowed for tracking of 
single glycine receptors for at least 20 min, which is 200 times longer than Cy3 dye. Also, due to their small dimensions, nanoparticles are able to access dense synaptic regions and provide dynamical analysis that cannot be achieved with the use of $500 \mathrm{~nm}$ latex beads, one of the probes typically used for studying single molecule properties in live cells.

Besides single molecule studies of neurotransmitters, QDs have been used to study neurotransmitter localization and signal pathways. Nanocrystals conjugated with peptides, antibodies, or other small molecules have been shown to recognize their target cell surface receptors ${ }^{25,38-40}$. It has also been shown that nanocrystal probes, after binding to their targets, can modulate receptor functions by either inhibiting ligand transportation ${ }^{39}$ or activating downstream signaling ${ }^{25,40}$.

Rosenthal and co-workers used nanocrystals conjugated with the neurotransmitter serotonin to target serotonin transporters on transfected cells ${ }^{39}$. Serotonin labeled nanocrystals specifically interacted with the serotonin receptor, and also inhibited the transportation of free serotonin in a way similar to antagonists.

Recently, Mason and coworkers studied norepinephrine (NE) and dopamine (DA) transporter (NET and DAT) locations and activities with semiconductor nanocrystal linked antibodies and peptides ${ }^{38}$. With streptavidin-biotin interaction, nanocrystals can specifically bind to NET in transfected cells as well as surface protein Limbic Associated Membrane Protein (LAMP) in hippocampal cultures. Since the activity of Ang II receptor is closely correlated with NET, the authors studied the localization of this receptor using nanocrystal-neuropeptide Ang II conjugates. This approach would allow for future study of Ang II receptor redistribution and dynamics in relation to NET activity in real-time. 
To investigate whether QDs can serve as fluorescent nano-devices to evoke specific cell physiological responses, $\mathrm{Vu}$ et al. linked the beta subunit of neuron growth factor ( $\beta \mathrm{NGF}$ ) to the nanocrystal surface and used this complex to target tyrosine kinase A (TrkA) receptors of PC12 cells ${ }^{40}$. They reported that nanocrystal- $\beta$ NGF activated TrkA receptor initiated downstream signaling that resulted in conversion of PC12 cells to a neural phenotype.

These experiments show that QD-ligand conjugates are promising imaging probes for studying receptor-mediated activities and will have a wide range of applications in pharmaceutics and therapeutics.

\subsection{Toxicity of Semiconductor Nanocrystals}

Cadmium and selenium are known to be toxic ${ }^{24}$. Therefore, concerns have arisen about semiconductor nanocrystal toxicity and their environmental impact. Most of the above cell and animal experiments showed that when properly capped by both $\mathrm{ZnS}$ and hydrophilic shells, no obvious CdSe nanocrystal toxicity was observed under normal experimental conditions. Several groups have varied parameters such as synthesis, surface coating and incubation concentration to further investigate the potential toxicities of nanocrystals ${ }^{24,41-43}$. Cytotoxicity was observed when $\mathrm{Cd}^{2+}$ or $\mathrm{Se}^{2+}$ ions were released. This occurred when the nanoparticle surface coating was not stable, exposing the CdSe to oxidization by air or UV damage ${ }^{24,43}$. Surface molecules also play a role in QD

cytotoxicity ${ }^{42,43}$. While cells can tolerate PEG-silica coated QDs at concentrations up to $30 \mu \mathrm{M}$, mercaptopropionic acid coated QDs have deleterious effect at $\sim 6 \mu \mathrm{M}^{43}$. 
Besides cytotoxicity, the degradation and metabolism of nanocrystals in the body remains to be investigated and there are reports that injected nanocrystals can accumulate in kidney, liver and spleen ${ }^{32,33}$. Whether nanocrystals can ultimately be cleared from the body is not known. More research in this area must be completed before they can be used as probes for diagnostic applications.

\subsection{Developing New Nanoprobes from Semiconductor Nanocrystals}

In 2003, "Science News" commented that QDs "just might be the next stars of biological imaging". Indeed, in the past several years, there has been an enormous body of research dedicated to using QDs in a variety of biological applications. Although they will not replace traditional fluorophores in biological imaging, QDs have been gradually accepted as a better alternative probes with enhanced signal-to-noise, extremely high stability, and improved specificity suitable for studying important biological problems.

The focus of this dissertation is based on the development of quantum dots in biological imaging, and has been aimed to develop new probes that are even better than QDs alone. Specifically speaking, two strategies are applied. In one strategy, we take advantage of the increasing capability of manipulating the shape of semiconductor nanocrystals, and developed rod shaped semiconductor nanocrystals as fluorescent labels. Since high quality semiconductor nanocrystals, especially those with good shape control, are only soluble in organic solvents, surface modification is the first step and is very critical for the success of their biomedical applications. Chapter 2 is dedicated to the surface modification of nanocrystals and a general route by surface silanization is developed within the dissertation research. In chapter 3, the applications of 
semiconductor quantum rods in a few biological-imaging situations are demonstrated, which shows that quantum rods, with their unique optical properties, opens up many new possibilities in biological imaging. In another strategy, we developed new nanoprobes by assembling QDs and Au nanoparticles into discrete nanostructures using DNA. We then demonstrated using proof of principle experiments that these nanostructures can be applied in probing hydrolytic enzyme function. Because the center-to-center distance between QDs and $\mathrm{Au}$ in these nanostructures with clarified interactions is more than $50 \mathrm{~nm}$, a dramatic extension from the $10 \mathrm{~nm}$ distance range of FRET, these structures should be applicable in detecting long-range biomolecular interactions not possible with present techniques. The synthesis of QDs /Au-DNA nanostructures is described in chapter 4. Chapter 5 is dedicated to their optical property characterization and application in probing the activity of hydrolytic enzymes. 


\section{References:}

1. Michalet X, Pinaud F, Lacoste TD, Dahan M, Bruchez M, Alivisatos AP, Weiss S: Properties of Fluorescent Seminconductor Nanocrystals and their Applications to Biological Labeling. Single Mol. 2001, 2:261-276.

2. Dabbousi BO, Rodriguez-Viejo J, Mikulec FV, Heine JR, Mattoussi H, Ober R, Jensen $\mathrm{KF}$, Bawendi M: (CdSe)ZnS core-shell quantum dots: synthesis and characterization of a size series of highly luminescent nanocrystallites. J phys Chem B. 1997, 101:94639475.

3. Hines MA, Guyot-Sionnest P: Synthesis and Characterization of Strongly Luminescing ZnS-Capped CdSe Nanocrystals. $J$ Phys Chem 1996, 100:468-471.

4. Bruchez M, Moronne M, Gin P, Weiss S, Alivisatos AP: Semiconductor Nanocrystals as Fluorescent Biological Labels. Science 1998, 281:2013-2016.

5. Chan WC, Nie S: Quantum dot bioconjugates for ultrasensitive nonisotopic detection. Science 1998, 281:2016-2018.

6. Dubertret B, Skourides P, Norris DJ, Noireaux V, Brivanlou AH, Libchaber A: In vivo imaging of quantum dots encapsulated in phospholipid micelles. Science 2002, 298:1759-1762.

7. Han MY, Gao XH, Su JZ, Nie S: Quantum-dot-tagged microbeads for multiplexed optical coding of biomolecules. Nature Biotechnology 2001, 19:631-635.

8. Mattoussi H, Mauro JM, Goldman ER, Anderson GP, Sundar VC, Mikulec FV, Bawendi MG: Self-assembly of CdSe-ZnS quantum dot bioconjugates using an engineered recombinant protein. Journal of the American Chemical Society 2000, 122:12142-12150.

9. Qu LH, Peng XG: Control of photoluminescence properties of CdSe nanocrystals in growth. J Am Chem Soc 2002, 124:2049-2055.

10. Dahan M, Levi S, Luccardini C, Rostaing P, Riveau B, Triller A: Diffusion dynamics of glycine receptors revealed by single-quantum dot tracking. Science 2003, 302:442-445. 
11. Kim S, Lim YT, Soltesz EG, De Grand AM, Lee J, Nakayama A, Parker JA, Mihaljevic T, Laurence RG, Dor DM, et al.: Near-infrared fluorescent type II quantum dots for sentinel lymph node mapping. Nature Biotechnology 2004, 22:9397.

12. Fu AH, Micheel $\mathrm{CM}$, Cha J, Chang $\mathrm{H}$, Yang $\mathrm{H}$, Alivisatos AP: Discrete nanostructures of quantum dots/Au with DNA. $J$ Am Chem Soc 2004, 126:1083210833.

13. Gueroui Z, Libchaber A: Single-molecule measurements of gold-quenched quantum dots. Phys Rev Lett 2004, 93:166108.

14. Oh E, Hong MY, Lee D, Nam SH, Yoon HC, Kim HS: Inhibition assay of biomolecules based on fluorescence resonance energy transfer (FRET) between quantum dots and gold nanoparticles. $J$ Am Chem Soc 2005, 127:3270-3271.

15. Alivisatos AP: Perspectives on the physical chemistry of semiconductor nanocrystals. J Phys Chem 1996, 100:13226-13239.

16. Gao X, Yang L, Petros JA, Marshall FF, Simons JW, Nie S: In vivo molecular and cellular imaging with quantum dots. Curr Opin Biotechnol 2005, 329:1173-1177.

17. Arya H, Kaul Z, Wadhwa R, Taira K, Hirano T, Kaul SC: Quantum dots in bioimaging: revolution by the small. Biochem Biophys Res Commun 2005, 329:1173-1177.

18. Ballou B, Lagerholm BC, Ernst LA, Bruchez MP, Waggoner AS: Noninvasive imaging of quantum dots in mice. Bioconjugate Chemistry 2004, 15:79-86.

19. Chan WC, Maxwell DJ, Gao X, Bailey RE, Han M, Nie S: Luminescent quantum dots for multiplexed biological detection and imaging. Curr Opin Biotechnol 2002, 13:40-46.

20. Larson DR, Zipfel WR, Williams RM, Clark SW, Bruchez MP, Wise FW, Webb WW: Water-soluble quantum dots for multiphoton fluorescence imaging in vivo. Science 2003, 300:1434-1436.

21. Grecco HE, Lidke KA, Heintzmann R, Lidke DS, Spagnuolo C, Martinez OE, JaresErijman EA, Jovin TM: Ensemble and single particle photophysical proper-ties (TwoPhoton excitation, anisotropy, FRET, lifetime, spectral conversion) of commercial 
quantum dots in solution and in live cells. Microscopy Research and Technique 2004, 65:169-179.

22. Pinaud F, King D, Moore HP, Weiss S: Bioactivation and cell targeting of semiconductor $\mathrm{CdSe} / \mathrm{ZnS}$ nanocrystals with phytochelatin-related peptides. $\mathrm{J} \mathrm{Am}$ Chem Soc 2004, 126:6115-6123.

23. Michalet X, Pinaud FF, Bentolila LA, Tsay JM, Doose S, Li JJ, Sundaresan G, Wu AM, Gambhir SS, Weiss S: Quantum dots for live cells, in vivo imaging, and diagnostics. Science 2005, 307:538-544.

24. Derfus AM, Chan WCW, Bhatia SN: Probing the cytotoxicity of semiconductor quantum dots. Nano Letters 2004, 4:11-18.

25. Lidke DS, Nagy P, Heintzmann R, Arndt-Jovin DJ, Post JN, Grecco HE, JaresErijman EA, Jovin TM: Quantum dot ligands provide new insights into erbB/HER receptor-mediated signal transduction. Nature Biotechnology 2004, 22:198-203.

26. Kaul Z, Yaguchi T, Kaul SC, Hirano T, Wadhwa R, Taira K: Mortalin imaging in normal and cancer cells with quantum dot immuno-conjugates. Cell Res 2003, 13:503-507.

27. Xiao Y, Barker PE: Semiconductor nanocrystal probes for human metaphase chromosomes. Nucleic Acids Res 2004, 32:e28.

28. Gerion D, Chen FQ, Kannan B, Fu AH, Parak WJ, Chen DJ, Majumdar A, Alivisatos AP: Room-temperature single-nucleotide polymorphism and multiallele DNA detection using fluorescent nanocrystals and microarrays. Analytical Chemistry 2003, 75:4766-4772.

29. Chen F, Gerion D: Fluorescent CdSe/ZnS nanocrystal-peptide conjugates for long-term, nontoxic imaging and nuclear targeting in living cells. Nano Letters 2004, 4:1827-1832.

30. Brokmann X, Ehrensperger MV, Hermier JP, Triller A, Dahan M: Orientational imaging and tracking of single CdSe nanocrystals by defocused microscopy. Chemical Physics Letters 2005, 406:210-214. 
31. Yildiz A, Selvin PR: Fluorescence imaging with one nanometer accuracy: application to molecular motors. accounts of Chemical Research 2005, appears on Web.

32. Pellegrino T, Parak WJ, Boudreau R, Le Gros MA, Gerion D, Alivisatos AP, Larabell CA: Quantum dot-based cell motility assay. Differentiation 2003, 71:542-548.

33. Nisman R, Dellaire G, Ren Y, Li R, Bazett-Jones DP: Application of quantum dots as probes for correlative fluorescence, conventional, and energy-filtered transmission electron microscopy. Journal of Histochemistry \& Cytochemistry 2004, 52:13-18.

34. Gao X, Cui Y, Levenson RM, Chung LWK, Nie S: In vivo cancer targeting and imaging with semiconductor quantum dots. Nat Biotechnol 2004, 22:969-976.

35. Akerman ME, Chan WC, Laakkonen P, Bhatia SN, Ruoslahti E: Nanocrystal targeting in vivo. Proc Natl Acad Sci U S A 2002, 99:12617-12621.

36. Alivisatos AP, Gu W, Larabell C: Quantum Dots as Cellular Probes. Annual Review of Biomedical Engineering 2005, 7.

37. Voura EB, Jaiswal JK, Mattoussi H, Simon SM: Tracking metastatic tumor cell extravasation with quantum dot nanocrystals and fluorescence emission-scanning microscopy. Nature Medicine 2004, 10:993-998.

38. Stroh M, Zimmer JP, Duda DG, Levchenko TS, Cohen KS, Brown EB, Scadden DT, Torchilin VP, Bawendi MG, Fukumura D, et al.: Quantum dots spectrally distinguish multiple species within the tumor milieu in vivo. Nat Med 2005.

39. Morgan NY, English S, Chen W, Chernomordik V, Russo A, Smith PD, Gandjbakhche A: Real time in vivo non-invasive optical imaging using near-infrared fluorescent quantum dots. Acad Radiol 2005, 12:313-323.

40. Mason JN, Farmer H, Tomlinson ID, Schwartz JW, Savchenko V, DeFelice LJ, Rosenthal SJ, Blakely RD: Novel fluorescence-based approaches for the study of biogenic amine transporter localization, activity, and regulation. $J$ Neurosci Methods 2005, 143:3-25. 
41. Rosenthal SJ, Tomlinson A, Adkins EM, Schroeter S, Adams S, Swafford L, McBride J, Wang YQ, DeFelice LJ, Blakely RD: Targeting cell surface receptors with ligand-conjugated nanocrystals. Journal of the American Chemical Society 2002, 124:4586-4594.

42. $\mathrm{Vu}$ TQ, Maddipati R, Blute TA, Nehilla BJ, Nusblat L, Desai TA: Peptideconjugated quantum dots activate neuronal receptors and initiate downstream signaling of neurite growth. Nano Letters 2005, 5:603-607.

43. Hoshino a, Fujioka K, Oku T, Suga M, Sasaki YF, Ohta T, Yasuhara M, Suzuki K, Yamamoto K: Physicochemical properties and cellular toxicity of nanocrystal quantum dots depend on their surface modification. Nano Letters 2004, 4:2163-2169.

44. Shiohara A, Hoshino A, Hanaki K, Suzuki K, Yamamoto K: On the cyto-toxicity caused by quantum dots. Microbiol Immunol 2004, 48:669-675.

45. Kirchner C, Liedl T, Kudera S, Pellegrino T, Javier AM, Gaub HE, Stolzle S, Fertig N, Parak WJ: Cytotoxicity of colloidal CdSe and CdSe/ZnS nanoparticles. Nano Letters 2005, 5:331-338. 


\section{Chapter 2}

\section{Surface Modification of Colloidal Nanocrystals for Biomedical Applications}

\subsection{Introduction}

Colloidal nanocrystals are of great interests because of their unique properties. Besides the composition, shape and size, the surface characteristics are also extremely important to achieve desired optical, electronic, mechanical, catalytic and other functions. Specifically for nanocrystals targeting applications in medical biology field, with hydrophilic surface is one of the prerequisites. Even for nanoparticles already hydrophilic, the surface characteristics can still be the major determinants of how these nanocrystals interacting with biological systems. For example, surfaces of colloidal nanoparticles introduced into the body as advanced drug delivery tools play an important role in the clearance kinetics and biodistribution of these particles ${ }^{1}$.

Several studies have used surface modification and bioconjugation in the application of nanoparticles in biological detection or therapeutic function. In general, 
the surface modification involves coating nanoparticles with an additional layer, which renders these particles water soluble and at the same time provides different functional groups for further bioconjugation. This layer can be small bi-functional molecules, which covalently bind to the surface of nanoparticles ${ }^{2}$; or the layer may be micellar, which wraps the hydrophobic nanoparticles inside of their hydrophobic $\operatorname{cores}^{3}$; the use of amphiphilic polymer to coat nanoparticles is also reported as a general route, where the hydrophobic end of the polymer interacts with hydrophobic surfactant molecules on the nanoparticle surface ${ }^{4}$. Most of these studies have focused on surface modification of spherical particles. Whether these modification processes are affected by the morphology of nanoparticles has seldom been investigated. In this chapter, we will describe a silanization procedure that we designed for coating rod shaped semiconductor nanocrystals (quantum rods). Because quantum rods have larger surface strain, this makes surface modification much more challenging. The strain effect of rod shaped particles has been well demonstrated in the synthesis of core/shell quantum rods ${ }^{5}$, where a gradient shell of $\mathrm{CdS} / \mathrm{ZnS}$ has to be grown instead of simply a $\mathrm{ZnS}$ shell as for spherical semiconductor nanocrystals ${ }^{6}$. Indeed, as additional evidence that proves the bigger surface strain of rod shaped particles from a different angle, the new silanization procedure that works for coating quantum rods has been proven to be very robust and also works well for coating different types of colloidal nanocrystals.

\subsection{Surface Silanization of Semiconductor Quantum Rods CdSe/CdS/ZnS}


The most critical design in the silanization procedure for quantum rods is to use cross-linked silane molecules in the priming step of the coating, in contrast to the singlemolecule-priming as shown in silanization of spherical semiconductor nanocrystals ${ }^{7}$. The procedure is illustrated as following:

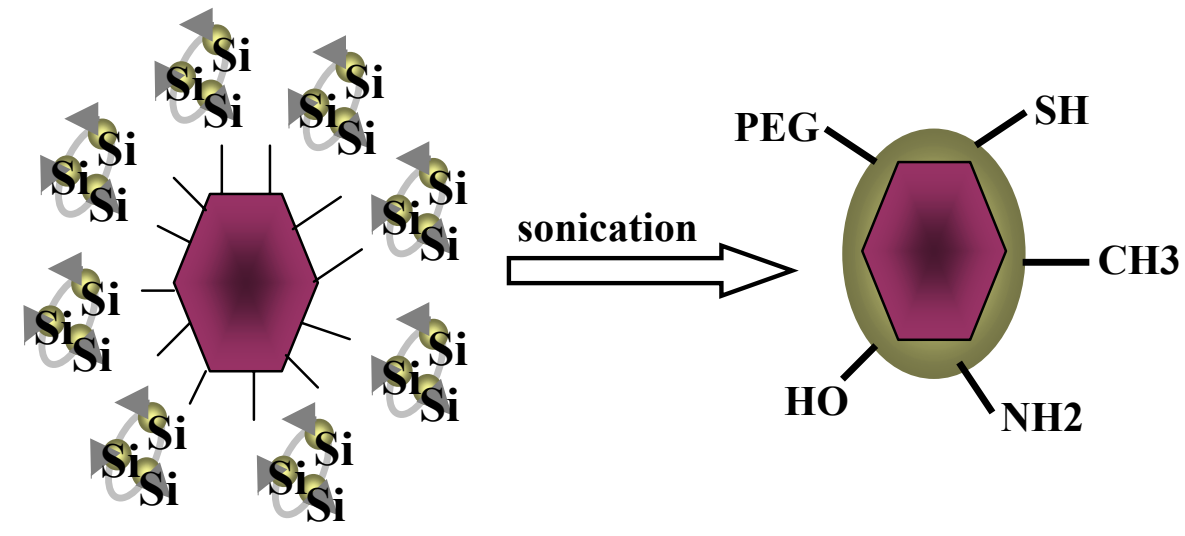

Figure 2.1 A cartoon to illustrate the surface silanization of nanoparticles. Cross linked silane molecules are used as priming agents, and different surface functional groups can be incorporated into silanization.

This can be realized by applying a reaction condition that favors condensation at the beginning of silanization. For a typical synthesis, starting from 500ul of quantum rods at sub-micromolar concentrations in chloroform, after precipitation using methanol, 200ul of mercapto-propyl trimethoxyl silane (mps) is added to the precipitates, immediately followed by addition of $1 \mathrm{ml}$ tetramethyl ammonia hydroxide (TMAOH). This mixture is then vortexed briefly and sonicated for $2 \mathrm{~h}$. It is worth noting that the base is in large excess and can ensure a basic condition even with large range of dilution. Through the above procedures, mps are linked to the surface of rods. At the same time, silane molecules are cross linked with each other by forming siloxane bond, making a very stable contacting layer of silane coating. The surface of quantum rods has already been changed from hydrophobic to hydrophilic up to this step, however, they are only soluble 
in basic water condition, once the $\mathrm{pH}$ of the solution is lowered, the particles will cross link with each other and form a piece of solid gel.

To make these particles stable under physiological condition, further surface modification is needed. So for the above solution after sonication, 800ul of PEG-silane, $2 \mathrm{ul}$ of mps and $36 \mathrm{ul}$ of $\mathrm{H}_{2} \mathrm{O}$ are added, then the solution is sonicated for another one and half hour. PEG molecules are reported to be very efficient molecules in rendering particles biocompatibility and can dramatically increase the duration of circulation of coated particles in vivo by reducing liver and spleen uptake ${ }^{1,8}$. Since PEG is a nonionic polymer, the effect of it on colloidal stability is known by steric stabilization ${ }^{1}$. The siloxane bond formed between the PEG-silane molecules and the already existing silane layer provide strong anchoring of the PEG molecules to the surface, desorption or free lateral movement of the molecule on the surface are prevented. PEG coated particles have good water solubility. Nonetheless, the surface modification has not finished up to this step, because there are still hydroxyl functional groups on the surface that may crosslink particles by forming inter particle siloxane bond. The interparticle crosslinking could lead to long-term instability of silanized particles and cause agglomeration.

To avoid the instability, a final step to quench the surface excess hydroxyl groups is added to silanization. Specifically speaking, $0.2 \mathrm{ml}$ chloro-trimethyl silane is added to $2 \mathrm{ml}$ of methanol, subsequently the mixture is added into $0.34 \mathrm{~g}$ of TMAOH powder, then the mixture is added into PEG coated particle solution. Afterwards, the solution is heated up to $60^{\circ} \mathrm{C}$ for half an hour, then left under stirring overnight. This step can also be 
accomplished by sonicating the solution for 2 hours, then leaving it to sit overnight, which makes the silanization procedure happen all-in-a-sonicator. The all-in-a-sonicator process is applied for coating the magnetic nanoparticles as described later. For semiconductor quantum rods and QDs, the quenching step is performed in the flask, since sonication of quenching solution leads to decreased quantum yield of the fluorescent nanoparticles.

The silanized quantum rod solution is condensed down to sub micro molar concentration using a Microcon centrifugal device. The resulting condensed solution can be easily dialyzed into neutral buffer. $10 \mathrm{mM}$ potassium phosphate buffer solution is used with $\mathrm{pH}$ of 7.3 for this purpose. The resulting quantum rods are stable for over 2 years and are proven to be very biocompatible by different biological labeling experiments as shown in Chapter 3.

\subsection{Surface Silanization of Semiconductor Quantum Dots CdSe/ZnS}

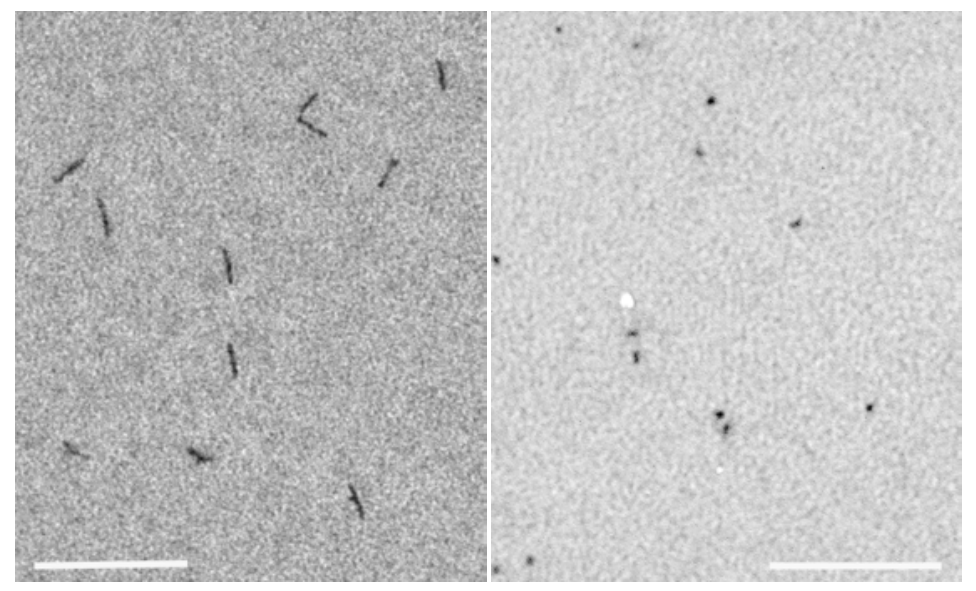

Figure 2.2 TEM images of silanized quantum rods (a) and QDs (b). Scale bars are 100nm. 
As mentioned earlier, quantum rods have bigger surface strain than quantum dots and are more difficult for surface modification ${ }^{5}$. Therefore, the procedure developed for coating quantum rods is readily applicable to coat the smaller spherical particles.

\subsection{Characterization of the Silanization Coating Thickness}

Figure 2.1 shows the TEM images of silanized quantum rods and QDs. The particles are coated by very thin silica shells that are difficult to detect via TEM.

One way to detect the silica shell using TEM is to apply particles on holy carbon TEM grid, where the particles are protruding out of the carbon support film so that part of the particles will have nothing underneath, hence, low contrast silica shell can be observed. An example is shown in figure 2.3, where the silica shell thickness have been grown thicker on purpose with addition of sodium silicate in the silanization procedure after the priming silanization coating step.

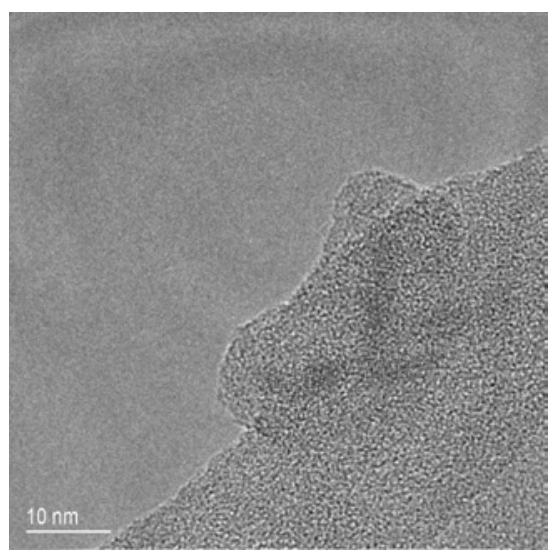

Figure 2.3 TEM of silanized quantum rods with thicker silica shell by addition of sodium silicate in the silanization procedure. 
However, for silanized quantum rods with thin silica coatings, such clear TEM images have not been acquired. Using TEM grids of Ted Pella ${ }^{\text {TM }}$ 01890-F, which is a grid with lacey carbon film and removable Formvar backing. This grid type is good for high definition imaging with no effects of underlying supporting material. However, it seems very hard to have rods protruding into the holes of the mesh; instead, the Formvar film is very easy to peel off and quantum rods tend to align with the edge of the film, as shown in Figure 2.4. From the distance between the edge of the film and the edge of the rod $\mathrm{ZnS}$ shell, it is evident that the silica shell is definitely smaller than $5 \mathrm{~nm}$ as shown in most of the images. And the shell thickness is possibly smaller than 2 or $3 \mathrm{~nm}$ as presented in some of the TEM images. By changing the grid type to 01824 of Ted Pella, which is made with ultra thin carbon film on a holy carbon support film, some rods are protruding of the carbon support film, however, the ultra thin carbon film covering the grid, though claimed by Ted Pella to provide practically no interference with specimen material imaged in the TEM, diminishes the contrast for the silanization coating, so no information of the thickness of silica shell can be concluded (Figure 2.4).

Better information about shell thickness comes when applying samples on the holy carbon TEM grids with Lacey/Carbon film from Electron Microscopy Sciences $(\mathrm{LC} 225-\mathrm{Cu})$. As shown in Figure 2.5, shell thickness of roughly $2.5 \mathrm{~nm}$ to $3 \mathrm{~nm}$ are observed. The thickness is clearly thinner than the thickness of silanization shell as shown in Figure 2.2, which proves that indeed, we can control the thickness of the silanization coating. 

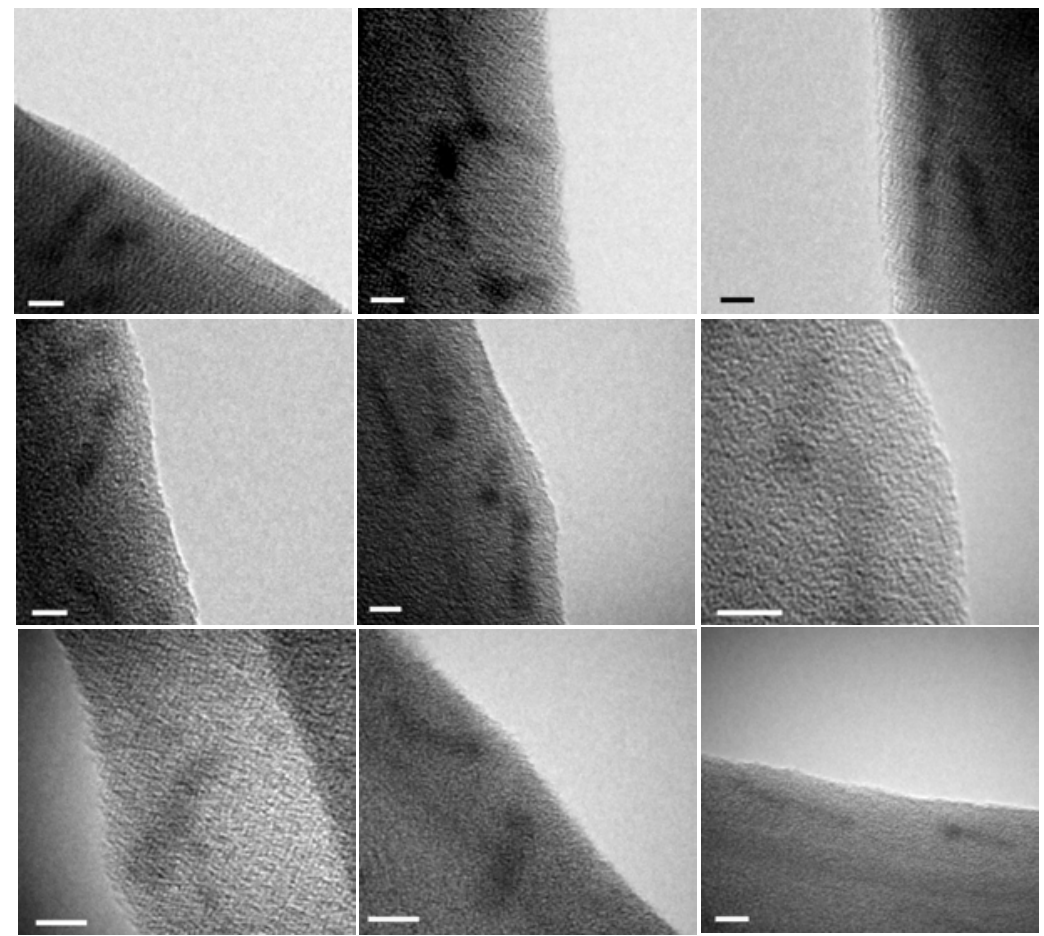

Figure 2.4 TEM images of silanized quantum rods on Ted Pella 01890-F TEM grids. Scale bars are $5 \mathrm{~nm}$.

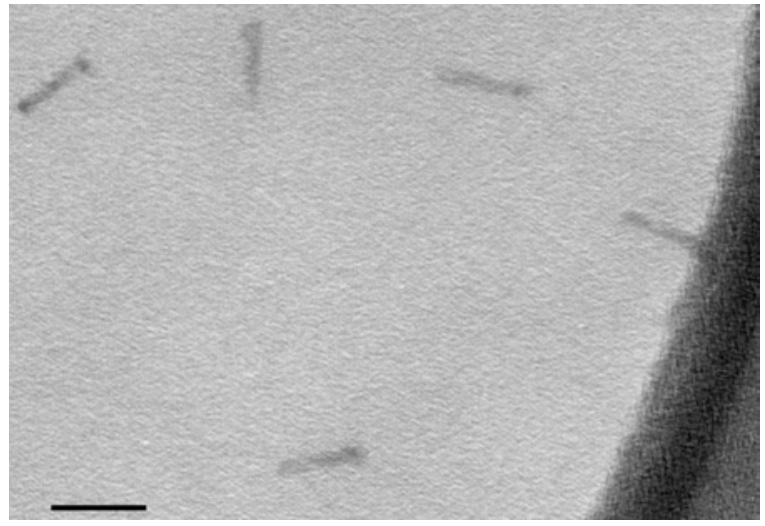

Figure 2.5 TEM of silanized quantum rods on Ted Pella 01824-F TEM grids. Scale bar is 20nm.

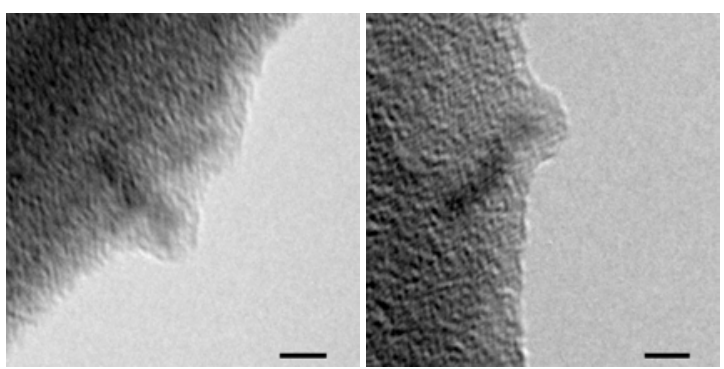

Figure 2.6 TEM of silanized quantum rods on Electron Microscopy Sciences LC225-Cu grids. Scale bars are $5 \mathrm{~nm}$. 
TEM detects physical size of the shell after particles dried on the TEM grid. The shell thickness can also be obtained from the difference between the effective sizes of particles before and after silanization coating using dynamic light scattering. Dynamic light scattering measures the time dependence of the light scattered from a small sample solution region over a specific time range. The fluctuations in the scattered light intensity and frequency are related to the diffusion rate of molecules in and out of the region. The data can be analyzed to give the diffusion coefficients of the particles that scatter the light and can be processed to give the particle sizes. For spherical particles, the relationship between the diffusion constant and particle sizes are based on Stokes-Einstein equation:

$$
D=\frac{k T}{6 \pi \eta r}
$$

Where $k=1.38 \times 10-23 \mathrm{~J} / \mathrm{K}$ is the Boltzaman constant, $\mathrm{T}$ is the absolute Temperature, $\eta$ is the viscosity of the solvent, and $\mathrm{D}$ is the diffusion constant, $\mathrm{r}$ is the hydrodynamic radius of the particle. For rod like molecules, the relationship between diffusion constant and dimensions of the particles is:

$$
D=\frac{A k T}{3 \pi \eta L}
$$

where $\mathrm{D}, \mathrm{T}, \mathrm{k}, \eta$ are defined in the same way as for spherical molecules, in addition, $\mathrm{L}$ is the length of the rod molecule, $\mathrm{d}$ is the diameter of the rod, and A is a correlation factor given as: 


$$
A=\ln (L / d)+0.312+\frac{0.565}{L / d}-\frac{0.1}{(L / d)^{2}}
$$

In principal, sizes of particles either in rod shape or in spherical shape can be measured by dynamic light scattering. However, the instrument used in this experiment, which is Malvern Instruments ZetaSizer NanoZS, can only give size information of spherical particles. So only sizes of QDs are measured. Figure 2.5 shows the results. For

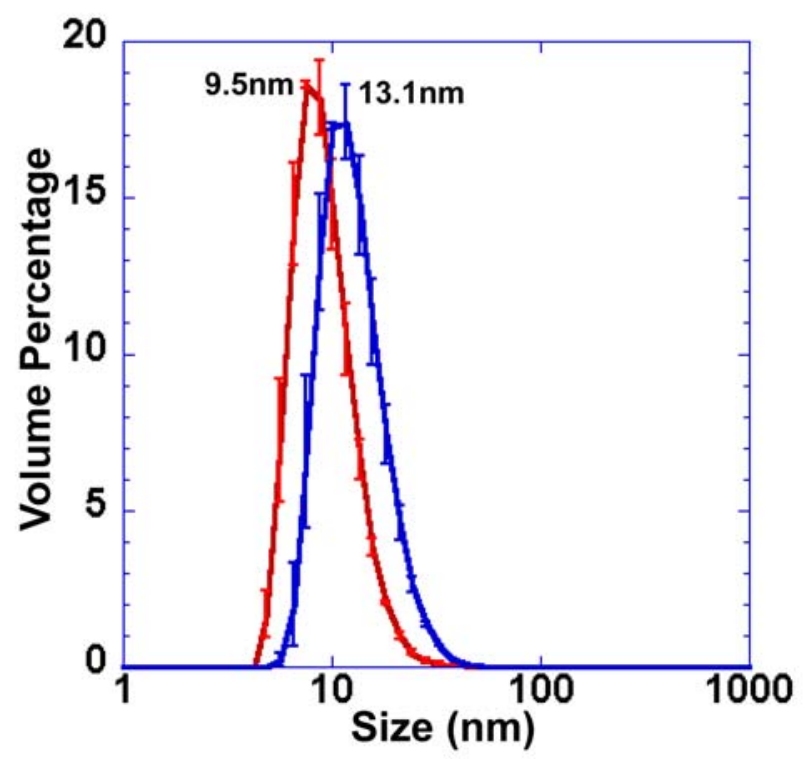

Figure 2.7 Sizes of CdSe/ZnS QDs in chloroform (red) and silanized CdSe/ZnS QDs (blue) in PB buffer measured using Malvern Instruments ZetaSizer NanoZS.

hydrophobic $\mathrm{CdSe} / \mathrm{ZnS}$ dots in chloroform, the mean diameter is $9.5 \mathrm{~nm}$, as shown by the red curve. For silanized $\mathrm{CdSe} / \mathrm{ZnS}$ in $\mathrm{PB}$ buffer, the mean diameter is $13.1 \mathrm{~nm}$, as shown by the blue curve. A difference of $3.6 \mathrm{~nm}$ is clearly observed, which corresponds to a silanization coating of $1.8 \mathrm{~nm}$. Since hydrophobic CdSe/ $\mathrm{ZnS}$ has a surfactant layer of trioctylphosphine oxide (TOPO) on the surface ${ }^{9}$, which contributes to the measured 
hydrodynamic diameter of rods in chloroform. Consequently, the real thickness of the silanization coating may be a little bit bigger than $2 \mathrm{~nm}$. This number is consistent with the TEM characterization (Figure 2.6) of quantum rods.

\subsection{Silanization of Metal Alloy and Metal Oxide Nanocrystals}

The silanization procedure developed for coating semiconductor quantum rods should also be applicable for coating other types of nanoparticles. For example, magnetic nanoparticles offer many attractive applications for biomedical and biological research. Before interesting biomedical applications of magnetic nanoparticles can be realized, they must first be rendered to be stable under physiological conditions. Although a variety of biocompatible molecules, including dextran, polyvinyl alcohol and phospholipids, have been used to coat the surface of magnetic nanoparticles and have proven successful for further diagnostic or therapeutic applications, general coating strategies are lacking. The only reported coating method that could be applied to different types of materials depends on an amphiphilic polymer layer, which adds more than $7 \mathrm{~nm}$ of coating thickness from hydrodynamic diameter measurement, resulting in much more bulky particles than silanization ${ }^{4}$. Hence, to test the generality of the silanization procedure, and at the same time to obtain biocompatible magnetic nanoparticles, we have applied silanization to chemically modify the surface of two different types of magnetic nanoparticles, one is metal alloy of FePt, the other is hollow metal oxide nanoparticles of $\mathrm{Fe}_{2} \mathrm{O}_{3}$. As expected, both samples are silanized well. Figure 2.8 shows TEM images of FePt and $\mathrm{Fe} 2 \mathrm{O} 3$ in neutral PB buffer. Furthermore, different surface functional groups can be introduced onto the surface by using different 
silane molecules for the outer layer. Shown in figure $2.8 \mathrm{~b}$ are $\mathrm{Fe}_{2} \mathrm{O}_{3}$ with a mercapto-rich surface, and in figure 2.8c are silanized $\mathrm{Fe}_{2} \mathrm{O}_{3}$ with an amino-rich surface.

(a)

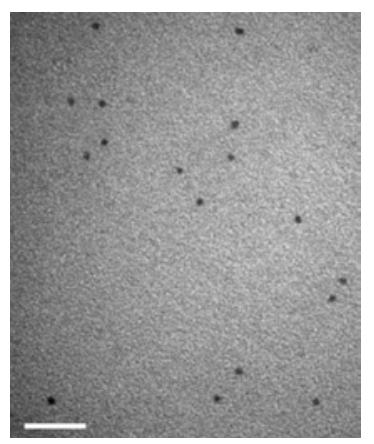

(b)

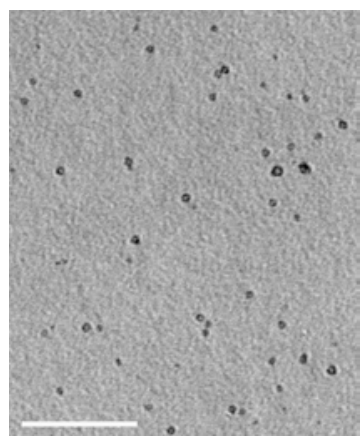

(c)

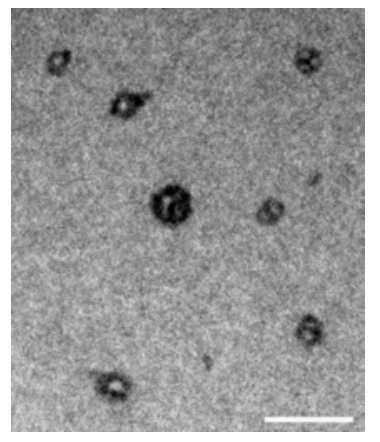

Figure 2.8 TEM images of silanized FePt (a), Fe2O3 with mercapto rich surfaces (b) and Fe2O3 with amino rich surfaces (c). Scale bars are $20 \mathrm{~nm}$ for a and c, and $100 \mathrm{~nm}$ for $\mathrm{b}$.

\subsection{Conclusions}

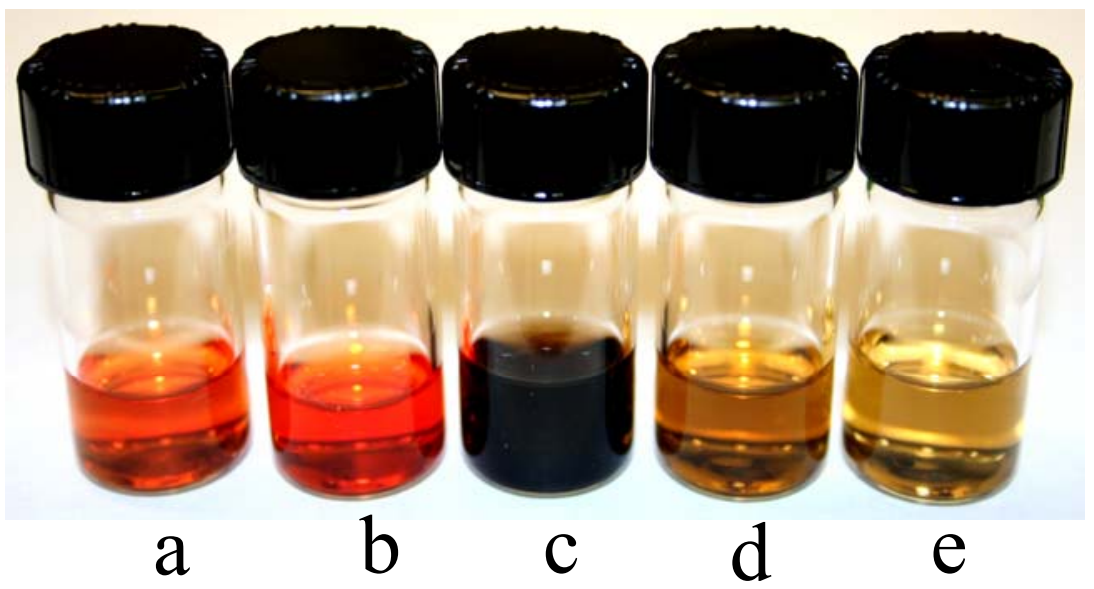

Figure 2.9 Pictures of different samples after silanization in neutral PB buffer. a, CdSe/CdS/ZnS rods; b, CdSe/ZnS dots ; c, FePt; d, Hollow $\mathrm{Fe}_{2} \mathrm{O}_{3}$ with amino rich surface; e, Hollow $\mathrm{Fe}_{2} \mathrm{O}_{3}$ with mercapto rich surface.

As shown in Figure 2.9, surface silanization developed here has been demonstrated to be able to coat nanoparticles with different shapes, and can be applied 
for coating different types of nanoparticles, including metal alloys, semiconductors and metal oxides. Furthermore, nanoparticles with different properties can go through the same silanization coating and retain the characteristics of their original property; therefore silanization modification can produce nanoparticles targeting different biomedical applications. For example, semiconductor nanocrystal quantum rods after silanization can be used as fluorescent biological labels, which are described in detail in chapter 3. The silanization procedure represents a general surface modification method for making water-soluble and biocompatible nanoparticles. 


\section{References:}

1. Storm, G., Belliot, S. O., Daemen, T., Lasic, D. D. Surface modification of nanoparticles to oppose uptake by the mononuclear phagocyte system. Advanced Drug Delivery Reviews 17, 31-48 (1995).

2. Chan, W.C.W., Nie, S. Quantum dot bioconjugates for ultrasensitive nonisotopic detection. Science 281, 2016-2018 (1998).

3. Dubertret, B., Skourides, P., Norris, D.J., Noireaux, V., Brivanlou, A. H., Libchaber, A. In Vivo imaging of quantum dots encapsulated in phospholipid micelles. Science 298, 1759-1762 (2002).

4. Pellegrino, T., Manna, L., Kudera, S., Liedl, T., Koktysh, D., Rogach, A. L., Keller, S., Radler, J., Natile, G., Parak, W. J. Hydrophobic Nanocrystals Coated with an Amphiphilic Polymer Shell: A General Route to Water Soluble Nanocrystals. Nano Lett. 4, 703-707 (2004).

5. Manna, L., Scher, E.C., Li, L.S. \& Alivisatos, A.P. Epitaxial growth and photochemical annealing of graded $\mathrm{CdS} / \mathrm{ZnS}$ shells on colloidal CdSe nanorods. Journal Of The American Chemical Society 124, 7136-7145 (2002).

6. Dabbousi, B.O., Rodriguez-Viejo, J., Mikulec, F. V., Heine, J. R., Mattoussi, H., Ober, R., jensen, K. F., Basendi, M. G. (CdSe)ZnS Core-Shell Quantum Dots: Synthesis and Characterization of a Size Series of Highly Luminescent Nanocrystallites. J. Phys. Chem. B 101, 9463-9475 (1997).

7. Gerion, D., Pinaud, F., Williams, S. C., Parak, W. J., Zanchet, D., Weiss, S., Alivisatos, A. P. Synthesis and Properties of Biocompatible Water-Soluble SilicaCoated CdSe/ZnS Semiconductor Quantum Dots. J. Phys. Chem. B 105, 88618871 (2001).

8. Gao, X., Cui, Y., Levenson, R. M., Chung, L. WK., Nie, S. In Vivo cancer targeting and imaging with semiconductor quanutm dots. Nature Biotechnology 22, 969-976 (2004).

9. Hines, M.A., Guyot-Sionnest, P. Synthesis and Characterization of Strongly Luminescing ZnS-Capped CdSe Nanocrystals. J. Phys. Chem. B 100, 468-471 (1996). 


\section{Chapter 3}

\section{Semiconductor Quantum Rods as Fluorescent Biological Labels}

\subsection{Introduction}

In recent years, semiconductor quantum dots have been applied with great advantage in a wide range of biological imaging applications ${ }^{1-4}$. The continuing developments in the synthesis of nanoscale materials and specifically in the area of colloidal semiconductor nanocrystals ${ }^{5-7}$ have created an opportunity to generate a next generation of biological labels with complementary or in some cases enhanced properties compared to colloidal quantum dots. In this paper, we report the development of rod shaped semiconductor nanocrystals (quantum rods) as new fluorescent biological labels. We engineered biocompatible quantum rods by surface silanization and applied them for non-specific cell tracking as well as specific cellular targeting. The very striking properties of quantum rods as demonstrated here are enhanced sensitivity and greater resistance for degradation as compared to quantum dots. Quantum rods have many 
potential applications as biological labels in situations where their properties offer advantages over quantum dots.

The challenges of biological imaging demand further development of new molecular probes and contrast agents that have better sensitivity, longer stability, good biocompatibility and minimum invasiveness. The convergence of nanotechnology and biotechnology has created many innovations to meet this challenge. A variety of different approaches in making new nanoprobes have been developed in recent years. For example, core/shell fluorescent silica nanoparticles were shown to be highly photostable in comparison to single organic dye molecules ${ }^{8}$; Noble metal nanoparticles have been reported as molecular rulers based on plasmon coupling ${ }^{9}$; Magnetic nanocrystals have been shown as effective contrast agents for magnetic resonance imaging ${ }^{10,11}$; and nanoparticle-based bio-bar codes were reported for ultrasensitive detection of proteins ${ }^{12}$. Among various nanomaterials developed, semiconductor nanocrystals, also known as quantum dots (QDs), represent one of the most successful new biological probes. Compared to conventional organic fluorophores, QDs have advantageous properties, including tunable emission, exceptional photostability, high multiplexing capability and extreme brightness ${ }^{1-4}$. Quantum dots are now commercially available and used in an everwidening array of biological applications.

The ability to manipulate the shape of semiconductor nanocrystals has led to rod shaped semiconductor nanocrystals, hereafter referred to as "quantum rods" $(\mathrm{QRs})^{5,13}$. QRs are semiconductor nanocrystals with diameters ranging from 2 to $10 \mathrm{~nm}$ and with 
lengths ranging from 5 to $100 \mathrm{~nm}$. The band gap of the rods depends strongly on diameter, but only weekly on length. Thus the emission can be readily tuned by diameter, but the absorption cross section can be chosen using the length. In addition to the properties inherited from quantum dots, such as size-tunable broad absorption and narrow symmetric emission, as well as extreme resistance to photobleaching, quantum rods have many unique properties that make them potentially better probes for some biomedical applications. For example, when compared to quantum dots, QRs have larger absorption cross section ${ }^{14}$, faster radiative decay rate ${ }^{15}$, bigger Stokes shift ${ }^{5}$, and can be functionalized with multiple binding moieties. Furthermore, a single quantum rod exhibits linearly polarized emission unlike plane-polarized light from a single quantum dot. The polarization is purely linear when the aspect ratio is above $3^{5}$. All of these properties are desirable for certain biological applications and bring new possibilities for biological labeling. However, due to the large surface strain intrinsic to rod shaped particles $^{13}$, it is more challenging for surface modification of quantum rods in order to transfer them from organic solvents to physiological buffer conditions. Therefore, there is little work reported about using quantum rods for biomedical imaging and detection. In this chapter, we report the use of $\mathrm{CdSe} / \mathrm{CdS} / \mathrm{ZnS}$ core/shell quantum rods as a new generation of biological label, and demonstrate that QRs can be used in a variety of bioimaging applications. QRs are longer than QDs, so for some applications they may prove too large. However, we found that they could be used in a surprisingly large number of situations. Further, for single molecule in vivo studies, they are much better than QDs.

\subsection{Surface Silanization of Quantum Rods}


Similar to QDs, high quality quantum rods as synthesized are only soluble in organic solvents. A variety of approaches can be used to render quantum dots or rods water soluble and biocompatible. Silanization is one of the most powerful, as the resulting particles are truly biocompatible and extremely stable in biological environments. We designed a robust coating method for surface silanization of core/shell quantum rods. To overcome the enhanced surface strain from a rod compared to a dot, silane molecules were added in the priming step under a condition that favored condensation (Figure 3.1a), enabling a well-coated rod surface compared to single-silanemolecule-priming as reported for the silanization of spherical quantum dots ${ }^{16}$. Moreover, most silanization steps were performed inside a sonicator with temperature control, promoting uniform coating and a highly reproducible process. The silanization procedure thus developed for quantum rods could be readily applied for making water-soluble quantum dots and other types of nanoparticles, representing a general method to modify surfaces of nanoparticles. Quantum rods after silanization were stable in aqueous buffer for over 2 years. Figure $3.1 \mathrm{~b}$ shows the transmission electron microscopy (TEM) images of silanized quantum rods in neutral phosphate buffer (PB). The absorption and emission spectra of silanized quantum rods (Figure 3.1c) show that the characteristic optical properties of CdSe core of the rods are kept, and are identical to the optical profiles of quantum dots ${ }^{16}$. 

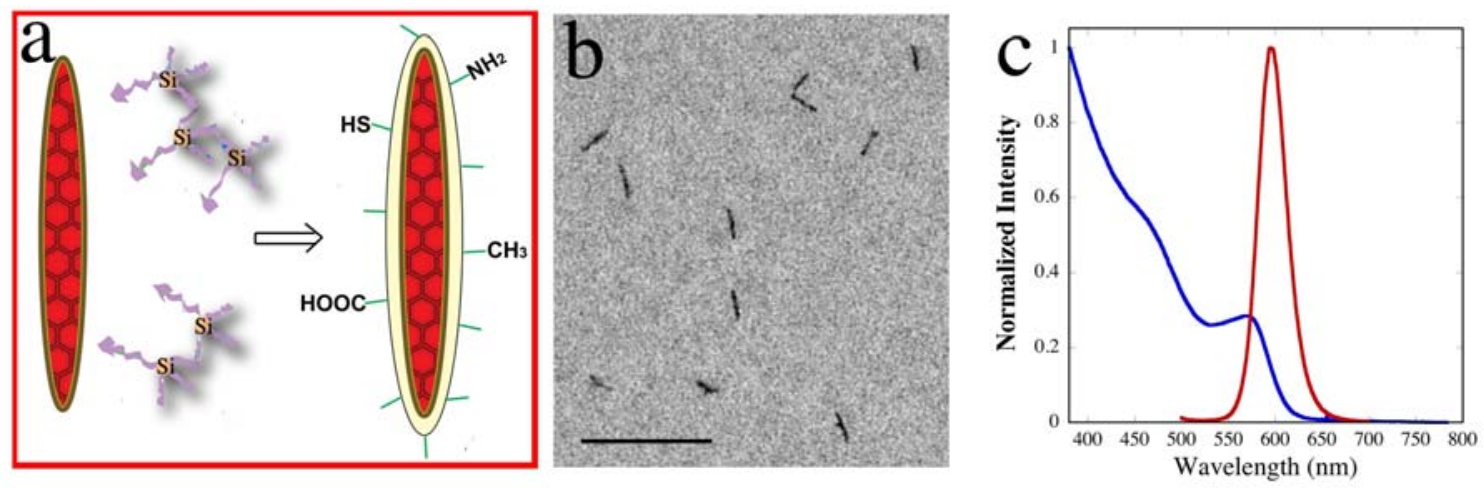

Figure 3.1 Synthesis and property characterization of Silanized Quantum Rods. (a) A cartoon illustrating silanization of quantum rods. Crosslinked silanes are priming molecules for the surface coating. (b) TEM image of silanized rods in neutral phosphate buffer. Scale bar $=100 \mathrm{~nm}$. (c) The UV-Vis absorption and emission spectra of silanized rods. The blue curve is the absorption spectrum, while the red curve is the emission spectrum.

\subsection{Silanized Quantum Rods are Biocompatible}

The silanized quantum rods are totally biocompatible. Previously, our group showed that when live cells were cultured on a layer of silanized quantum dots they uptook the nanoparticles and left behind a particle free trail, the area of which correlated with the metastatic potential of different cell lines ${ }^{17,18}$. Similarly, various live cells could also incorporate silanized quantum rods as they migrate on a layer of the nanocrystals, without influence on cell division and migration (see Figure 3.2). The good biocompatibility of quantum rods was also evidenced by direct delivery with Chariot ${ }^{\mathrm{TM}}$ 19, a peptide non-covalently interacting with quantum rods and transferring the cargo through the cell membrane (Figure 3.3). Quantum rods showed no apparent adverse effect on cells over the time period ( 24 hours) of our experiment. 
MDA-MB-231
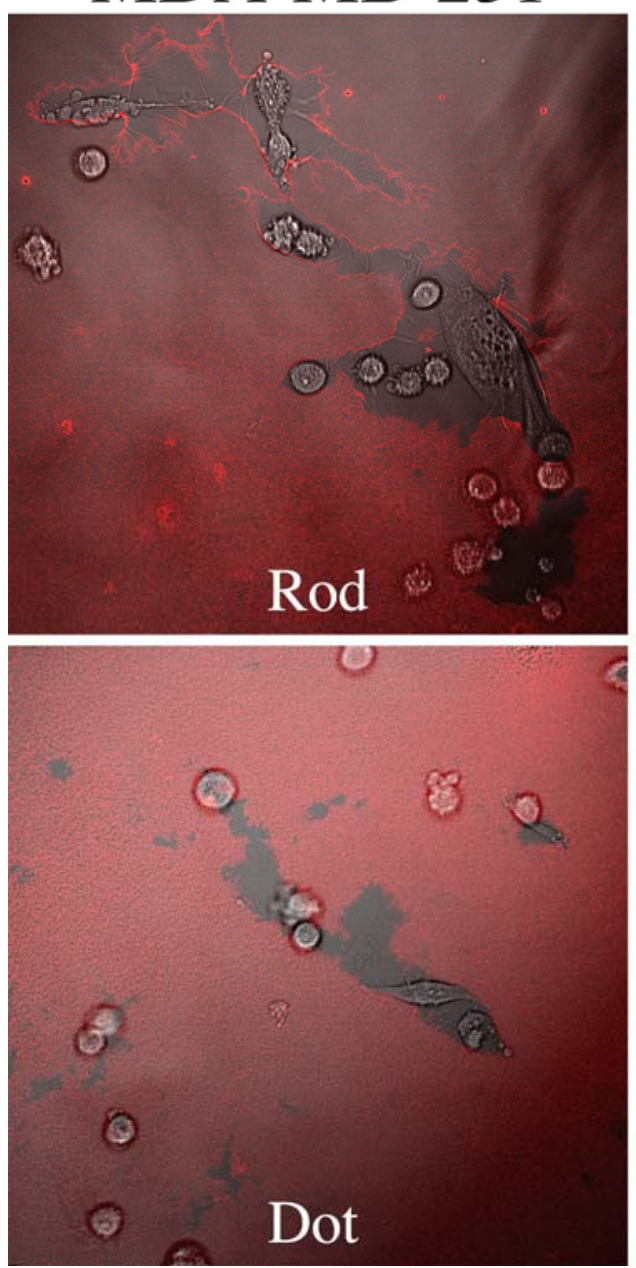

SW 480 Cells
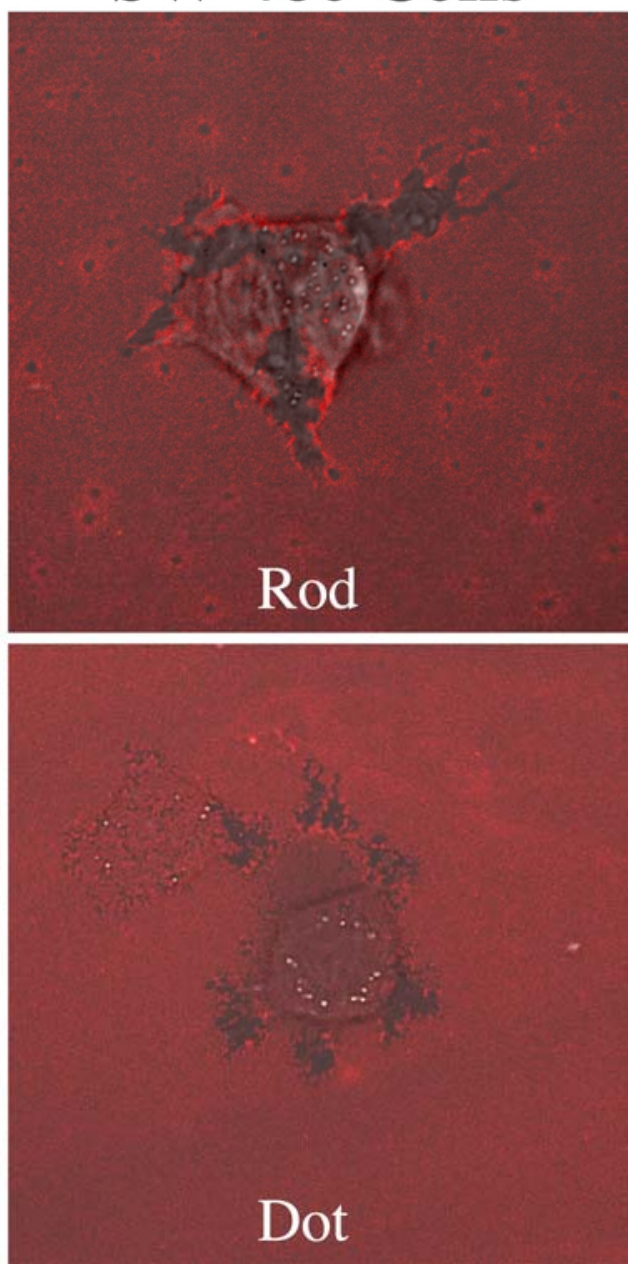

Figure 3.2 Human breast cancer cell MDA-MB-231 and colon cancer cells SW 480 cultured on top of quantum rod layer left fluorescence free area after 24 hours incubation. The size of the fluorescence free area is related to the invasiveness of the cancer cell, as being reported previously while cells were cultured on quantum dot layer. 


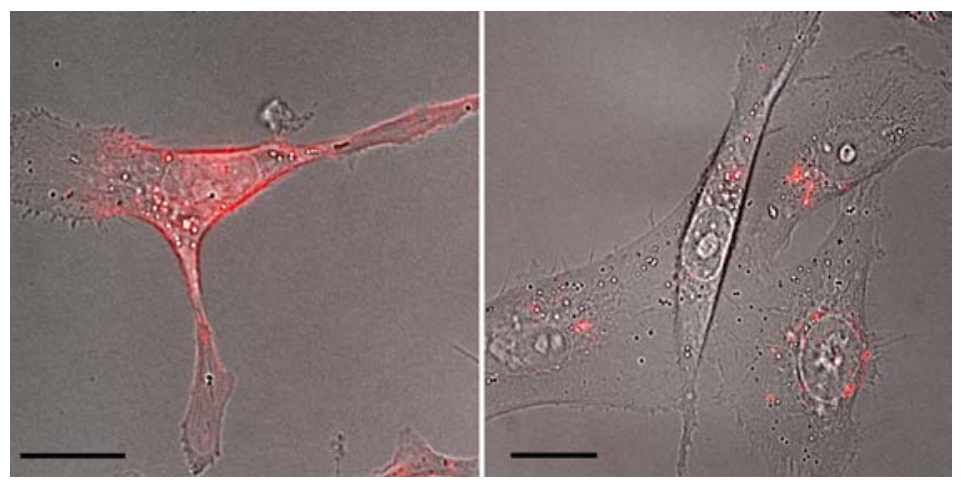

Figure 3.3 Silanized rods are biocompatible and not toxic to living cells. The red fluorescence in the images is from quantum rods in human breasts cancer cells MDA-MB-231 after $1 \mathrm{~h}$ (left) and 24h (right) transfected with Chariot ${ }^{\mathrm{TM}}$. These are merged images of transmission and fluorescent micrograms. Scale bar is $20 \mu \mathrm{m}$.

\subsection{Reduced Cd2+ Leakage of Silanized Quantum Rods Compared to Quantum Dots}

Although silanized quantum rods have shown little disturbance on cell function, potential cytotoxicity is a concern for these cadmium-based materials. Cytotoxicity was reported when $\mathrm{Cd}^{2+}$ or $\mathrm{Se}^{2+}$ ions were released from quantum dots ${ }^{2,3}$. Therefore, we

measured the $\mathrm{Cd}^{2+}$ leakage from both quantum rods and quantum dots by inductively coupled plasma optical emission spectroscopy (ICP/OES). Since ICP/OES measurements of $\mathrm{Cd}^{2+}$ concentration showed that same optical density (OD) of the nanocrystals at $488 \mathrm{~nm}$ corresponds to same amount of CdSe material in a sample (data now shown), we standardized the $\mathrm{Cd}^{2+}$ leakage from rod and dot samples by normalizing to the $\mathrm{OD}$ at $488 \mathrm{~nm}$ of the original samples. For rod and dot samples that were silanized under the 
same condition and stored at $4^{\circ} \mathrm{C}$ for 3 months, the $\mathrm{Cd}^{2+}$ concentration in the solution after nanocrystals being filtered were analyzed. $\mathrm{Cd}^{2+}$ presented in the solution that was filtered from the rod sample was less than one third of that filtered from the dot sample (Figure 3.4). This demonstrated that $\mathrm{Cd}^{2+}$ leakage was dramatically reduced by making

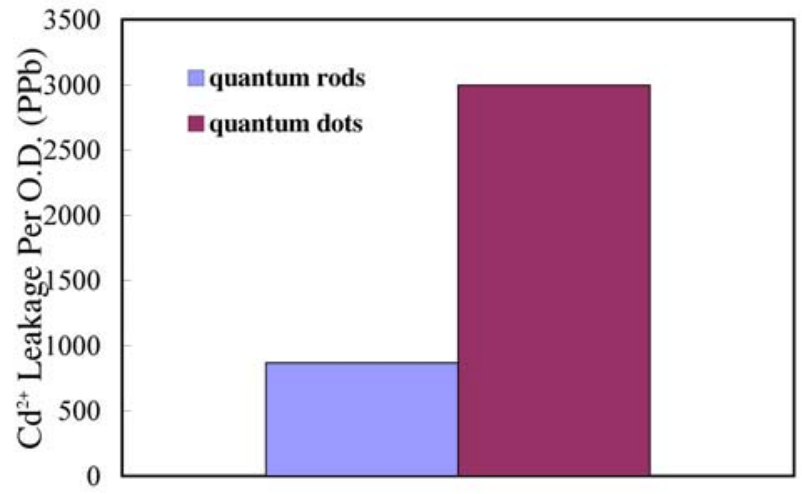

Figure 3.4 Rod shaped nanocrystals reduce the $\mathrm{Cd}^{2+}$ leakage significantly over that of spherical nanocrystals. The $\mathrm{Cd}^{2+}$ leakage was assayed by ICP/OES.

the same amount of CdSe material into a rod shape versus that of a spherical shape. The decrease in $\mathrm{Cd}^{2+}$ leakage of quantum rods was partially due to reduced ratio of surface area over volume (A simple calculation will result in a reduction factor of 1.25 from dots to rods with nanocrystal geometries as shown in the TEM images of Figure 3.5). The most important contribution to the reduction of quantum rod $\mathrm{Cd}^{2+}$ leakage arose from the reduced curvature effect in a rod shaped particle over that of a spherical particle, hence the rod surface was more resistant to such corrosion process as photooxidation. It is worth to mention that multiple studies both in vivo and in vitro have shown no noticeable adverse effects from QDs ${ }^{18,20,21}$. The enhanced resistance to degradation of quantum 
rods compared to quantum dots makes them an even less concern for cytotoxicity due to $\mathrm{Cd}^{2+}$ leakage, and could extend the range of their potential biological applications.
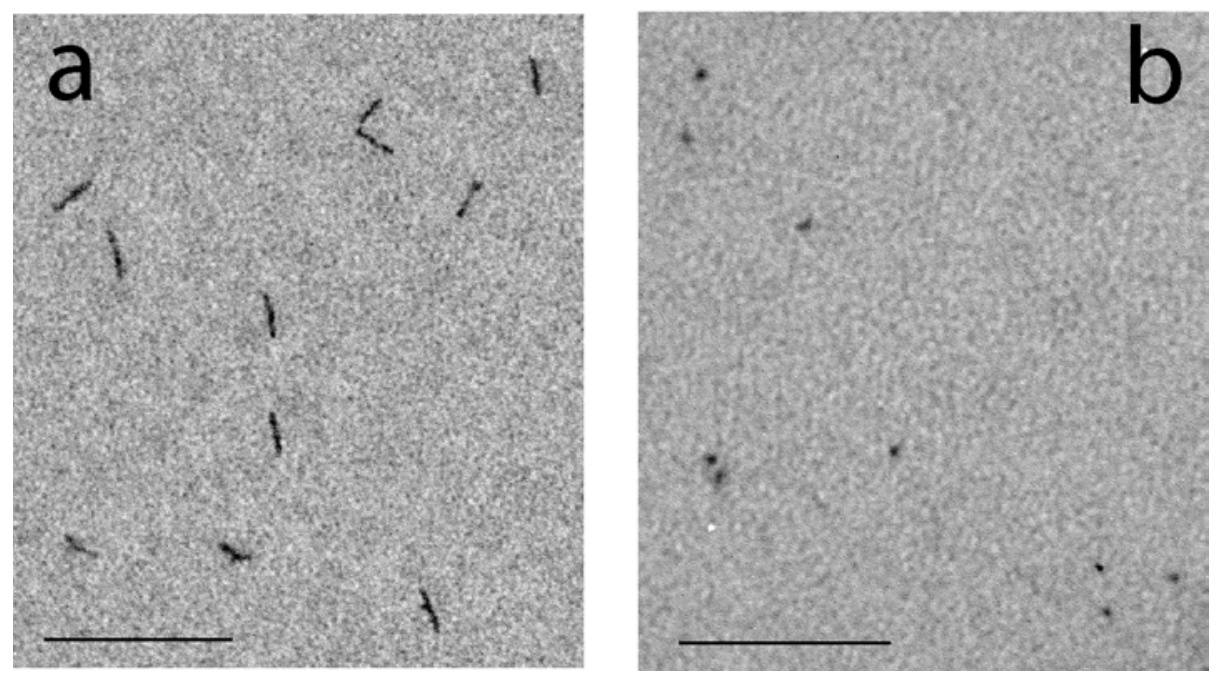

Figure 3.5 TEM images of quantum rods (a) and QDs (b) used in the Cd2+ leakage comparation. Scale bars are $100 \mathrm{~nm}$.

\subsection{Quantum Rods for Immunofluorescence Labeling}

As mercapto, amino, carboxyl and phosphonate functional groups could be easily incorporated into the design of surface coating by silanization, silanized quantum rods can be conjugated with various biomolecules to achieve precise biological functions. Antibody-antigen affinity is one of the most specific biological interactions and widely used for fluorescence imaging. We tested the conjugation of silanized particles with mercapto surface groups to amino bearing antibodies through a cross linker sulfo-SMCC as schematized in Figure 3.6. Conjugation with either whole antibody $\operatorname{IgG}$ or antibody 
fragments were achieved and evidenced by delay of the mobility of conjugates in gelelectrophoresis. To compare the specific cellular labeling efficiency of quantum rods with quantum dots (both have a quantum yield of 9\%), we picked a well-demonstrated system, that is, cancer cell marker Her2 on the surface of human breast cancer cell line SK-BR$3^{22}$, for specific labeling test. After incubating the cells with mouse anti-Her2 antibody
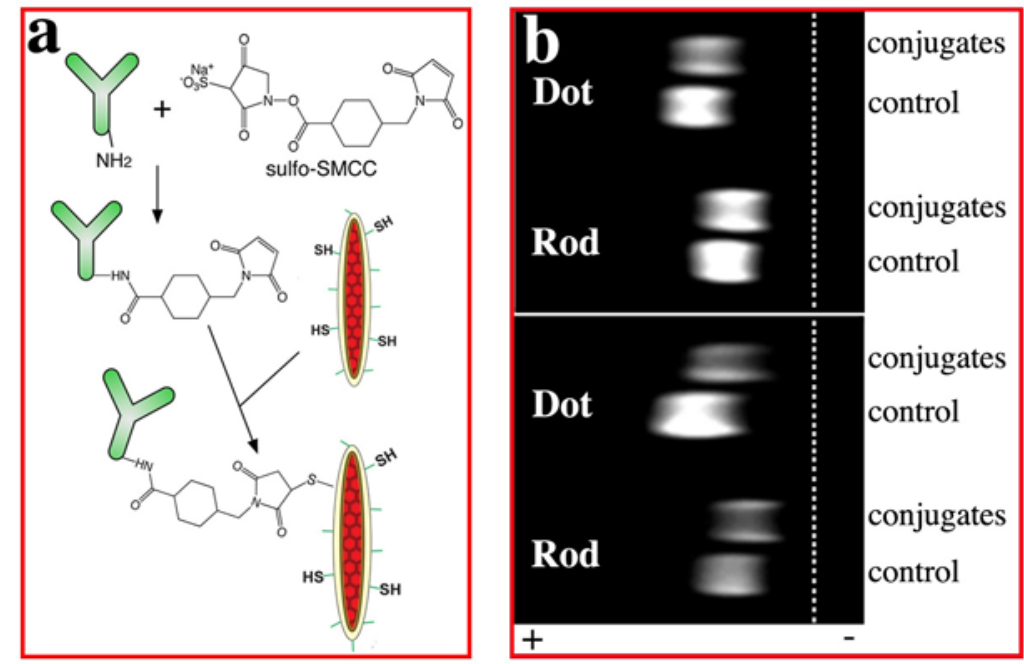

Figure 3.6 (a) The scheme for antibody bioconjugation of quantum rods with surface mercapto functional groups. (b) Electrophoresis analyses of quantum rods/dots bioconjugation. Top, quantum rods/dots conjugated with $\mathrm{F}\left(\mathrm{ab}^{\prime}\right)_{2}$ fragment of goat anti-mouse IgG antibody. Bottom, quantum rods/dots conjugated with whole goat anti-mouse IgG antibody. The conjugates moved slower than the free nanocrystals (control) due to the linkage with antibodies.

that binds to the external domain of Her2, we added quantum rod-goat anti-mouse $\mathrm{F}(\mathrm{ab} \text { ' })_{2}$ and quantum dot-goat anti-mouse $\left.\mathrm{F}(\mathrm{ab})_{2}\right)_{2}$ conjugates with the same $\mathrm{OD}$ at $488 \mathrm{~nm}$.

Specific targeting of the conjugates to cancer marker Her2 was clearly observed in both cases. It is worth mentioning that since the OD of quantum rods is the same as dots, the concentration of rods is only about $1 / 8$ of that of dots. However under such condition, the 
detected staining signal from quantum rod conjugates is as bright as that from dot conjugates (Figure 3.7), which indicates that quantum rods are more sensitive probes than quantum dots. This is expected because quantum rods have bigger absorption cross section than quantum dots at the same excitation wavelength ${ }^{14}$. At the same time, quantum rods are predicated to have faster radiative decay rates ${ }^{15}$, which correspond to a increased number of excitation and emission cycles within a signal collection period.
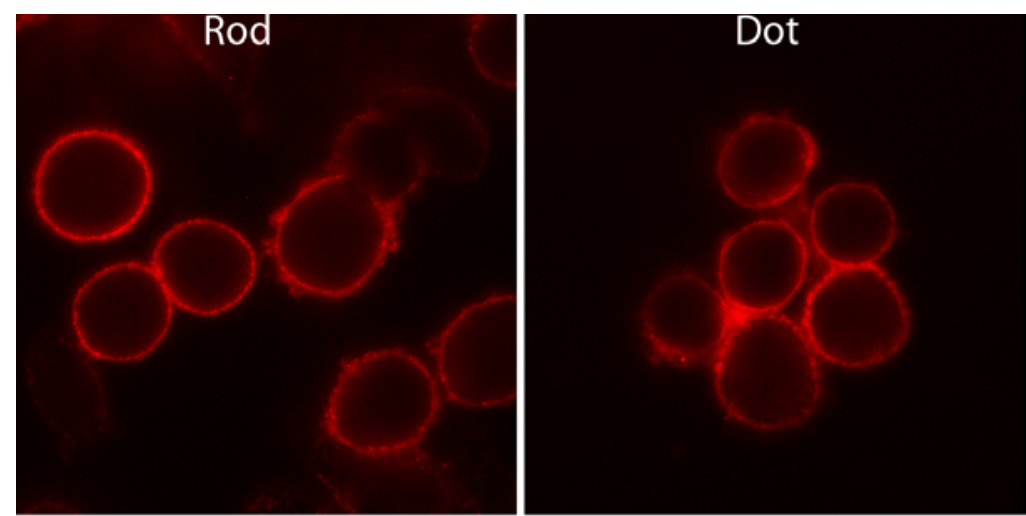

\section{Rod Control}

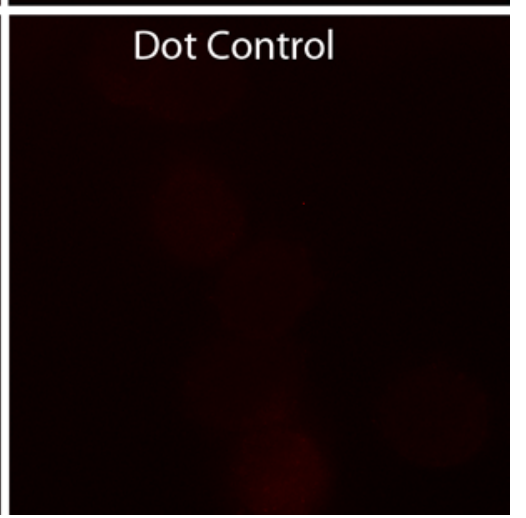

Figure 3.7 Immunofluorescence labeling of breast cancer cell marker Her2 on breast cancer cells SK-BR-3. The Her2 marker was labeled with mouse anti-Her2 antibody and goat anti-mouse IgG $\mathrm{F}\left(\mathrm{ab}^{\prime}\right)_{2}$ conjugated quantum rods/dots. The bottom images show that there is minimum binding of free nanocrystals to the anti-Her2 antibody treated cells. Scale bar is $20 \mu \mathrm{m}$.

\subsection{Quantum Rods for Single Molecule Imaging}


Since increasing the number of labeling particles can also enhance the signal intensity for ensemble fluorescent labeling, the increased detection signal from quantum rods is advantageous but not exceptional in ensemble systems. The more attractive turnout from the brighter fluorescence of quantum rods is for single molecule fluorescent imaging. The ability to track single molecules is a powerful method to study the dynamic and kinetic behavior of biomolecules inside living cells. Although quantum dots were shown to be able to image single molecules in living cells ${ }^{23,24}$, the enhanced fluorescent signal from quantum rods makes them ideal probes for single molecule tracking. We compared the fluorescence signals of quantum rods and quantum dots at the single molecule level as evidenced by blinking. Under the same excitation and detection conditions, the absolute fluorescent intensities of quantum rods were greatly improved from that of quantum dots (Figure 3.8a-b). To quantitatively compare the fluorescent signals, both rod and dot images were analyzed by automatically collecting fluorescent signals from a 15-frame image sequence using a self-written Matlab program. Figure 3c shows the number of particles picked up through the program as a function of threshold intensity and threshold image number. Threshold intensity is the intensity value set to differentiate signal from background in the program, and threshold image number defines that a signal has to appear at least in a certain number of images to be picked up as a particle, since particles are expected to blink, whereas noise pixels should not. In the plot of QRs, with increasing threshold and threshold image number, the number of quantum rods holds basically an island of stability where there is a clean distinction between particles and noise. However, for quantum dots, the number of particles decreases very quickly and it is very difficult to distinguish between particles and noise. This is because 
as compared to QRs, QDs are not as bright so they are not statistically distinct from the noise and thus not quite as accurate a measurable quantity as the rods. To quantitatively compare the intensity of QRs and QDs, We extract the particle intensities at threshold intensity 10 and threshold image number 5 - one of the points in the island of stability of QRs. Histograms of intensity distribution of both QRs and QDs are plotted as inserts in Figure 3.8c. The mean intensity is 29.5 for QRs and 12.7 for QDs, proving QRs are much more intense fluorescent probes than QDs. Under this condition, only 883 QDs are selected compared to 1624 particles for QRs. So we are in fact comparing the intensities from the brighter portion of quantum dots with almost a whole body of QRs, which may contain some low emission rods from possibly defects or surface imperfections during growth ${ }^{25}$. Further improvements in synthesis giving an intensity ratio of rods to dots close to theoretical value of 8 should be possible and will show a more dramatic advantage for QRs in single molecule probing. Moreover, in Figure 3C the number of particles of QDs goes down much faster with increasing threshold image number than QRs, meaning QDs blink more or have longer off times than QRs. This is consistent with previous report that blinking arises because the dot radiative rate is slower than a non-radiative mechanism ${ }^{25}$. Quantum rods have faster radiative deccay rate ${ }^{15}$, which may decrease the frequency of blinking thus yield a much better probe.

To demonstrate the ability of detecting and tracking of single quantum rods within living cells, we introduced small amount of silanized quantum rods to human breast cancer cell line MDA-MB-231 by the use of streptolysin-O (SLO), a bacterial protein 


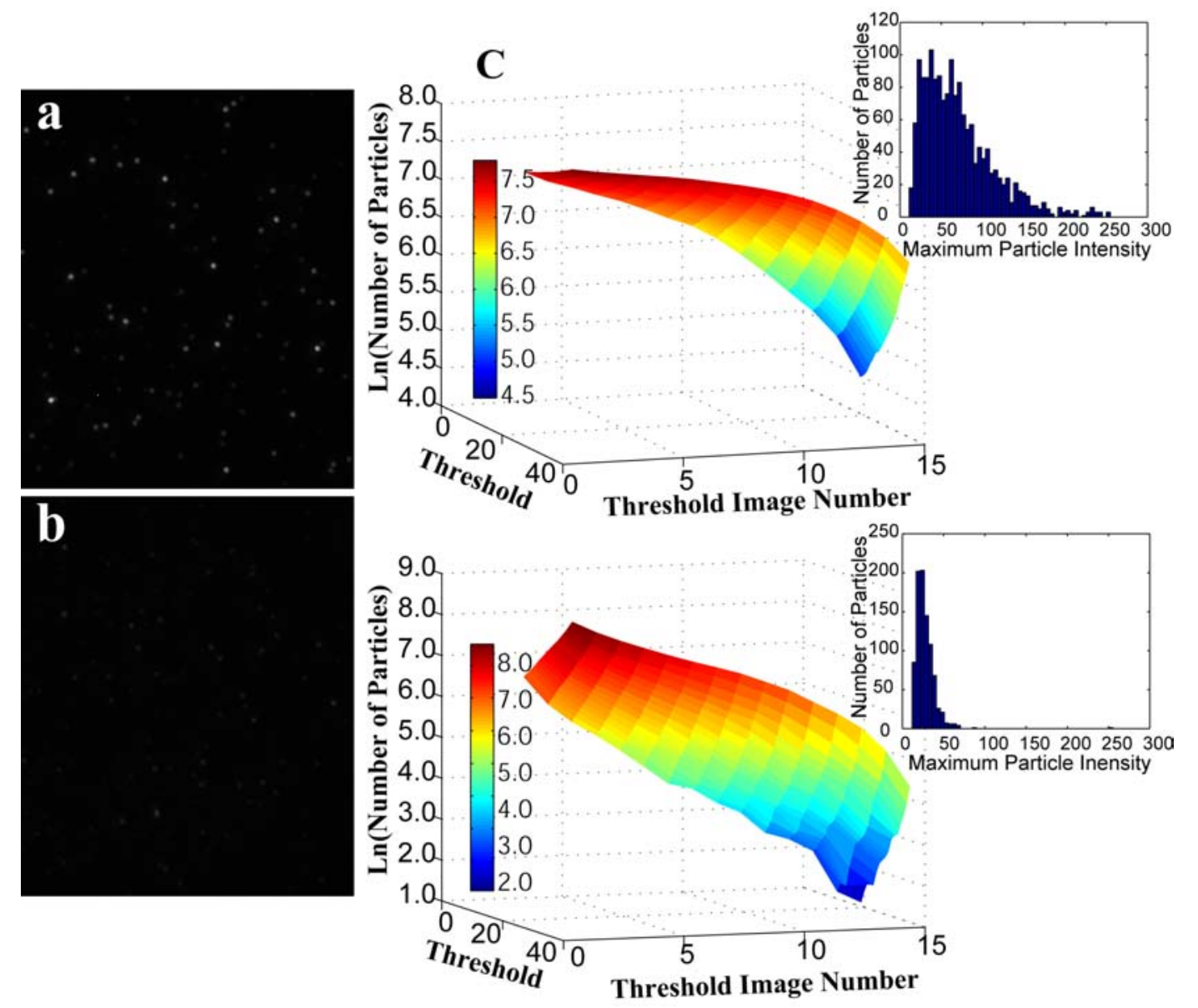

Figure 3.8 Fluorescence microscope images show that at single molecule level, quantum rods (a) are much brighter than quantum dots (b). (c) Statistical results of rods (top) and dots (bottom) from 15 image sequences. The color plots are the Ln of number of particles at different threshold intensity and threshold image number (the number of images that one particle at least appears in the 15 image sequence, for example, using a threshold image number 5 selects all the particle appears in at least 5 images of the 15 image sequence.) The Ln(Number of Particles) plots clearly illustrate how hard it is to different particles from background noise for QDs, as one can see that the particle number decreased all the way down dramatically when the threshold and threshold image number are increased. However, for the rod case, there is a broad region where the number of rod particles is relatively the same with increasing threshold and threshold image number. The inserts are the histograms of particle intensity distribution when using threshold 10, and threshold image number 5, which pick up 1624 rods giving a mean intensity of 29.5, and pick up 883 dots 
with a mean intensity of 12.7. Please note the much smaller number of particles picked up for QDs. In another word, only bright dot particles with relatively long on time of dot are considered in the statistics.

that binds to cholesterol and forms holes in the plasma membrane of animal cells ${ }^{26}$. Quantum rods retained their brightness inside living cells with good S/N (Figure 3.9). The tracking of single molecules was proved by particle blinking. As rod sizes get bigger,

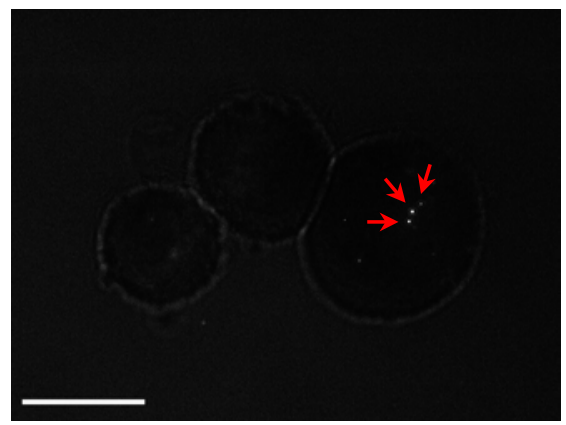

Figure 3.9 Single rods (indicated by arrows) are still very bright inside live MDA-MB-231 human breast cancer cells. Scale bar is $10 \mu \mathrm{m}$.

it may interfere with the molecular events that it intent to characterize, hence caution must be taken when time comes to interpret the data and a balance has to be found between the enhanced properties of quantum rods and the disadvantages in terms of their bigger sizes. However, this should not become an intrinsic limitation for single molecule tracking using QRs, as much bigger particles ${ }^{27}$, have been successfully applied in single molecule investigations.

\subsection{Quantum Rods as Single Molecule Orientation Probes}

Measuring structural dynamics of biomolecules is very important to understand biological mechanisms in cellular process. Investigations of dynamic biological systems 
such as actin and myosin interaction would benefit from new probes like QRs with more intense and polarized fluorescence (Figure 3.10).

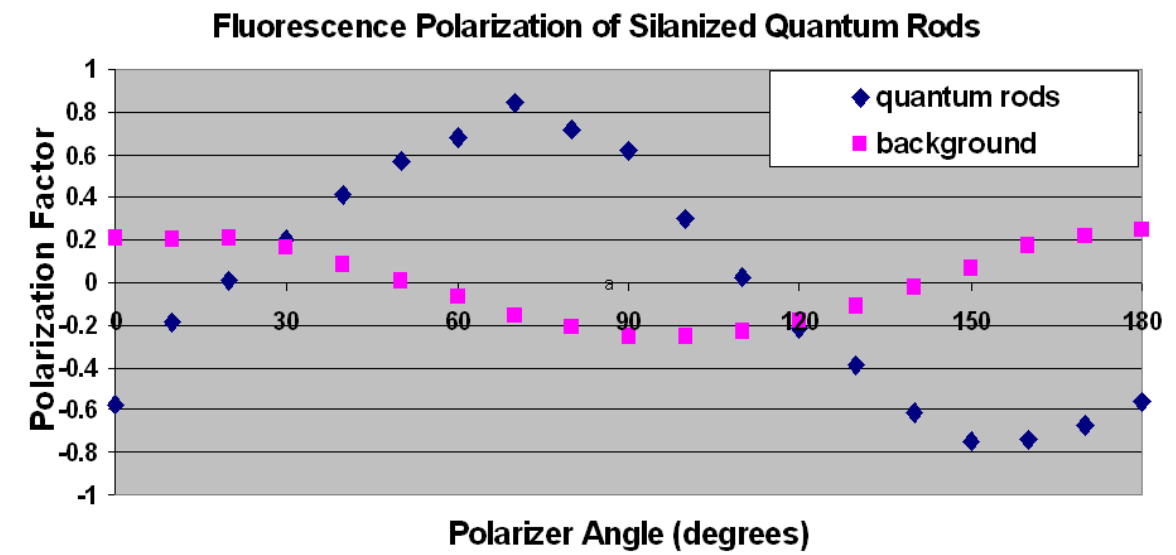

Figure 3.10 Polarized emission from single silanized quantum rods.

Actin is a cytoskeleton protein. Polymerized actin filaments provide mechanical support for cells, determine cell shape, and enable cell movements. Along with myosin, they play an essential role in muscle contraction or vesicle transportation. Polarized fluorescence microscopy has been used to study the orientation and dynamics of myosin, which reflects the population of several biochemical states of the myosin/actin interaction. However these studies are mostly based on traditional organic fluorophores, hence are limited in the observation time range, or they rely on more complicated optical set-up and analytical techniques.

Based on quantum rods' superior photostability and brightness, as well as the ability to give orientation information through linearly polarized emission, we believe that quantum rods can be used as fluorescence orientation probes to study this cellular process in vitro. We propose to conjugate the myosin to the surface of quantum rods. In 
order to study the dynamics of single myosin molecule, the stoichiometry of conjugation will be carefully controlled so that only one quantum rod per myosin, and idealy, through multiple linkage. This can be achieved by adjusting the relative concentrations of quantum rods, the crosslinker, and myosin. In vitro polymerized fluorescent labeled actin filaments will be attached to the substrate. Their interaction with myosin will be monitored with quantum rods. A CCD camera with beam splitting crystal will be used to detect the polarization of quantum rods, which should reflect the orientation status of myosin as it 'walks' along the actin filaments. The orientation information obtained will be compared with current reported model of the myosin/actin interaction.

Experiments like this should demonstrate proof of principal concept about applying quantum rods in biological molecule orientation probing. Based on this, more complicated molecular and cellular detection systems could be investigated utilizing the linearly polarized emission from quantum rods.

\subsection{Conclusions}

The introduction of biocompatible semiconductor quantum dots in $1998{ }^{28,29}$ has led to tremendous advances in biotechnologically important applications, including multiplexed in vivo imaging ${ }^{30,31}$, long term single molecule tracking ${ }^{23}$, deep tissue imaging and imaging guided surgery ${ }^{32}$, as well as hybrid inorganic-bioreceptor based optical sensing ${ }^{33}$. In this paper, we have described the development of rod shaped semiconductor nanocrystals for biological imaging. We have overcome the difficulty of rod surface modification and successfully transferred the nanocrystals from organic solvent to biological aqueous solutions by a silanization process. Silanized quantum rods 
have good biocompatibility and are potentially less cytotoxic than QDs. After further biofunctionization, quantum rods can be used as immunofluorescent probes. Compared to quantum dots, quantum rods are more intense and brighter probes, which was demonstrated clearly in single molecular imaging. The unique properties of quantum rods including distinctive shape, large absorption cross section, fast radiative decay rate, big stokes shift, multiple binding moieties and linearly polarized emission are yet still waiting to be fully exploited. We anticipate biocompatible quantum rods with properties superior to organic fluorophores and spherical quantum dots will have a very beneficial impact in many aspects of biomedical imaging and detection schemes.

\subsection{Methods}

Materials. Dimethylcadmium $\left(\mathrm{Cd}\left(\mathrm{CH}_{3}\right)_{2}, 97 \%\right)$ and tri-n-butylphosphine (TBP, 99\%) were purchased from Strem. Selenium (Se, 99.999\%), tri-n-octylphosphine oxide (TOPO, 99\%), diethylzinc (1.0M solution in heptane), hexamethyldisilathiane ((TMS) $\left.)_{2} \mathrm{~S}\right)$, tetramethylammonium hydroxide (TMAOH, pentahydrate $97 \%$, or $25 \%(\mathrm{w} / \mathrm{w})$ solution in methanol), (3-mercaptopropyl) trimethoxysilane (MPS, 95\%), chlorotrimethylsilane (CTS, 99\%) was purchased from Aldrich. Hexylphosphonic acid (HPA, 99\%) was purchased from Organometallics Inc. Tetradecylphosphonic acid (TDPA, 98\%) was purchased from Alfa. 2-[Methoxy(polyethylenoxy) propyl] trimethoxysilane (PEG-silane, 90\%) was purchased from Gelest. Potassium phosphate (PB, monobasic or dibasic) was purchased from Sigma. UltraPure ${ }^{\mathrm{TM}}$ agarose was purchased from Invitrogen. 
Synthesis of quantum rods. $\mathrm{CdSe} / \mathrm{CdS} / \mathrm{ZnS}$ quantum rods were synthesized following the published procedure ${ }^{13}$. All procedures were performed using standard air-free techniques. For CdSe core rods, $0.5 \mathrm{~g}$ of $\mathrm{Cd}\left(\mathrm{CH}_{3}\right)_{2}$ in TBP (32.99\% by weight) and $2.56 \mathrm{~g}$ of Se in TBP (7.78\% by weigth) were added to a mixture of $3.53 \mathrm{~g}$ of TOPO, $0.3 \mathrm{~g}$ TDPA and $0.08 \mathrm{~g}$ HPA. Nanocrystals were growing at $300^{\circ} \mathrm{C}$ for $7 \mathrm{~min}$. The sample was washed and dried under nitrogen, then stored in a glove box for shell growth with no further size selective precipitation. A $\mathrm{CdS} / \mathrm{ZnS}$ gradient shell was grown by injecting $2 \mathrm{ml}$ of chloroform solution of CdSe rods with a concentration of $1 \mathrm{~g} / \mathrm{L}$ into $5 \mathrm{~g}$ of TOPO. After pumping out all the chloroform, $0.5 \mathrm{ml}$ of $\mathrm{CdS} / \mathrm{ZnS}$ stock solution from a mixture of $2.057 \mathrm{~g}$ of TBP, $0.041 \mathrm{~g}$ of $\mathrm{Cd}\left(\mathrm{CH}_{3}\right)_{2}, 0.503 \mathrm{~g}$ of diethylzinc and $0.078 \mathrm{~g}$ of $(\mathrm{TMS})_{2} \mathrm{~S}$ was injected dropwise at $160^{\circ} \mathrm{C}$ and reacted for $10 \mathrm{~min}$. The resulting $\mathrm{CdSe} / \mathrm{CdS} / \mathrm{ZnS}$ core/shell rod solution was mixed with $3 \mathrm{ml}$ of octanol and stored in the dark inside glovebox.

Surface modification of quantum rods. A $1 \mathrm{ml}$ aliquot of $\mathrm{CdSe} / \mathrm{CdS} / \mathrm{ZnS}$ core/shell rods was precipitated using methanol, followed by addition of $200 \mu 1$ MPS. The sample was vortexed and then $1 \mathrm{ml} \mathrm{TMAOH}$ was added. The resulting solution was sonicated at $65^{\circ} \mathrm{C}$ for $2 \mathrm{~h}$. Afterwards, a dialysis solution of $450 \mu \mathrm{l}$ methanol and $1400 \mu \mathrm{l}$ of TMAOH was prepared, and $6 \mathrm{ml}$ of it was directly mixed with the former rods solution. The mixture was dialyzed for $1 \mathrm{~h}$, inside a Spectra/Por membrane (MWCO 25,000) tube (Spectrum Laboratories Inc). Next, $2 \mu 1$ MPS, 36 $\mu 1 \mathrm{H} 2 \mathrm{O}, 900 \mu 1$ PEG-silane was added and the sample was sonicated at $65^{\circ} \mathrm{C}$ for $1.5 \mathrm{~h}$. The sample was then transferred into a $50 \mathrm{ml}$ flask under $\mathrm{Ar}_{2}$. With vigorous stirring, $0.1 \mathrm{ml} \mathrm{CTS}, 2 \mathrm{ml}$ methanol and $0.32 \mathrm{~g}$ of solid TMAOH 
was added, followed by immediate heating of the sample to $60^{\circ} \mathrm{C}$ for $30 \mathrm{~min}$. The sample was kept stirring at room temperature overnight, and concentrated with Microcon YM100 filters. The concentrated sample was dialyzed in $1 \mathrm{~L}$ of $10 \mathrm{mM}$ PB (pH 7.3) overnight. Afterwards, the sample was filtered through MILLEX $^{\circledR}-G V 0.22 \mu \mathrm{m}$ filter unit (Millipore), and stored at $4^{\circ} \mathrm{C}$ in a refridgerator.

Quantum rods or quantum dots antibody conjugation. $2 \mathrm{mg}$ of Sulfo-SMCC was added into $0.15 \mathrm{ml}$ of $\mathrm{F}\left(\mathrm{ab}^{\prime}\right)_{2}$ fragment $(0.2 \mathrm{mg})$ goat anti-mouse $\operatorname{IgG}(\mathrm{H}+\mathrm{L})$ (Jackson ImmunoResearch), or whole goat anti-mouse $\operatorname{IgG}(\mathrm{H}+\mathrm{L})$ (0.3mg) (Jackson ImmunoResearch), and reacted on a vortexer foam rack for $1 \mathrm{~h}$. The sample was then run through a NAP 5 column to remove unreacted sulfo-SMCC, with $50 \mathrm{mM} \mathrm{PB}(\mathrm{pH} 7.3)$ as an elution buffer. Subsequently $51 \mu 1$ of quantum rods (OD $488 \mathrm{~nm} 0.11753$ ) or $20 \mu 1$ quantum dots (OD 488nm 0.30016) was mixed with $260 \mu 1$ of sulfo-SMCC labeled antibody and $49 \mu 11 \mathrm{M} \mathrm{NaCl}$. Then, $31 \mu 110 \mathrm{mM}$ PB (pH 7.3) was added into the dotantibody solution to render the solution volume the same as that of rod-antibody solution. Conjugating reaction solution was left on a vortexer foam rack for $2 \mathrm{~h}$ at room temperature. Next, conjugates were washed using Microcon YM-100 filter. Afterwards, an aliquot of conjugates were analyzed by $3 \%$ agarose gel electrophoresis under a voltage of $10 \mathrm{~V} / \mathrm{cm}$ (Bio-Rad). The remainder of conjugates was stored at $4^{\circ} \mathrm{C}$ in a refrigerator overnight before immunolabeling. 
Quantum rods characterizations. Optical absorbance was measured on an HP-8453 UV-Vis spectrophotometer (Hewlett-Packard). Fluoresence was measured using a SPEX Fluorolog-3 spectrofluorometer (Jobin Yvon horiba). TEM was measured on a FEI TECNAI $\mathrm{G}^{2}$ microscope under $200 \mathrm{keV} \cdot \mathrm{Cd}^{2+}$ concentration was analyzed on a Perkin Elmer 5300 DV Optical Emission ICP.

Cell culture. MDA-MB-231 and SK-BR-3 cells were obtained from ATCC. Cells were cultured in the appropriate media as following: MDA-MB-231 cells in Leibovitz's L-15 (ATCC) supplemented with 10\% fetal bovine serum (Gibco); SK-BR-3 cells in McCoy's 5A medium (ATCC) plus 10\% fetal bovine serum.

Cellular updake of nanocrystals. For Chariot ${ }^{\mathrm{TM}}{ }^{19}$ mediated quantum rods uptake, the cells were subcultured in 8-well chambered cover glass slides (LabTEK) pre-coated with collagen (Vitrogen) at a density of 10,000 cells per well. $80 \mathrm{ng} / \mathrm{ml}$ Chariot and $2 \mathrm{nM}$ silanized Quantum Rods (in PBS) were incubated at room temperature for 30 min. Cell medium was aspirated. Immediately after washing the cells with warm PBS, $50 \mu \mathrm{L}$ of Chariot-quantum rods mixture was added to each well, followed by $100 \mu \mathrm{L}$ serum free medium. The cells were incubated at $37^{\circ} \mathrm{C}$ in the tissue culture incubator for $1 \mathrm{~h}$ and then $250 \mu \mathrm{L}$ of medium supplemented with $16 \%$ serum was added to each well. The cells were either imaged right away or left in the incubator for later observation. For Streptolysin O (SLO, sigma) mediated quantum rod uptake, the procedure was modified according to literature ${ }^{26}$. In general, cells were trypsinized and washed twice with serum 
free medium. About 4 millions cells were then incubated with $0.5 \mathrm{nM}$ quantum rods and $40 \mathrm{U} / \mathrm{ml} \mathrm{SLO}$ at $37^{\circ} \mathrm{C}$ for $20 \mathrm{~min}$. The transfection was stopped by adding complete growth medium and incubated for another $20 \mathrm{~min}$. Cells were washed in complete growth medium twice and put on cover glass for imaging, or sub-cultured in 8-well chambered cover glass slides for later observation.

Immunofluorescence labeling. Cells were fixed in 4\% paraformaldehyde (Ted Pella) in cytoskeleton buffer (CSK: $100 \mathrm{mM} \mathrm{KCl,} 3 \mathrm{mM} \mathrm{MgCl}$, $5 \mathrm{mM}$ PIPES, $150 \mathrm{mM}$ sucrose, $\mathrm{pH}$ 6.8) at room temperature for $30 \mathrm{~min}$. Cells were rinsed in Superblock (SB, Pierce) (5 $\min x$ 3), then incubated in a solution of 1:100 anti-human, 1:100 anti-mouse Fab fragments (Jackson ImmunoResearch), and 10\% goat serum (GS, Gibco), in SB for 30 minutes to block non-specific labeling. After rinsing, cells were incubated in mouse antiHer2 antibody (1:30, zymed) in $\mathrm{SB}+10 \% \mathrm{GS}$ for $1 \mathrm{~h}$ while rocking at room temperature. They were washed (5 min $x 3$ ) with phosphate buffer saline (PBS) and incubated in goat anti-mouse conjugated quantum rods or dots (OD 0.0613 at $488 \mathrm{~nm}$ with $0.2 \mathrm{~cm}$ pass length) in PBS for $1 \mathrm{~h}$, and then washed again (5 $\min \times 3$ ) in PBS and ready to be observed. Control cells were treated with primary antibody ani-Her2, however for the secondary antibody-labeling step, unconjugated quantum rods/dots were added.

Fluorescence microscopy. A Zeiss AxioVert 200M fluorescence inverted microscope with a 103-watt mercury lamp and an AxioCam MRm CCD camera was used. Fluorescence signal was detected using either a Cy3.5 filter set (zeiss, exciter: BP 565/30, emitter: BP 620/60) or a QDot 605 filter set (chroma, exciter: E460SPUV, emitter: 
D655/20m). For detection of single molecules, $2 \mu \mathrm{L}$ of $0.6 \mathrm{nM}$ quantum rods, or $2 \mu \mathrm{L}$ of $5 \mathrm{nM}$ quantum dots were deposited on cover glass, dried using a compressed air blower, and then imaged with the microscope using a $60 \mathrm{X} 1.4$ NA oil immersion lens. For detection of single molecules inside the cells, the MDA-MB-231 cells were first loaded with small amounts of quantum rods with the use of SLO (see above part of cellular uptake of nanocrystals), and imaged with a 60X 1.4 NA oil immersion lens. For other fluorescence microscope experiments, a 40X 1.2 NA water immersion lens were used.

Statistical analysis. A Matlab program was written to analyze the single particle fluorescence images. First, all images were averaged into a single image and the particle positions were determined by selecting the brightest pixels in the conjoined image. Further, the most intense pixels corresponding to single particle fluorescence are selected by three criteria: (i) an average minimum distance between bright pixels, (ii) an average minimum intensity, (iii) a minimum number of images that the bright pixel appears in. The average minimum threshold distance was chosen to be five pixels. This criteria was chosen based on the conjoined image and the pixel distance necessary to distinguish between single particles and to select only a single pixel if there appeared to be a cluster of bright pixels. The average minimum intensity is a threshold intensity that is greater than that of the background such that only pixels distinguishable from the background are selected. In addition, since single particles blink whereas noise or bad pixels will tend not to blink or will appear in only a single image, a threshold image number is used to distinguish noise from particles. The intensities of each particle are then tracked in each subsequent image. With a threshold distance set to five, a threshold image number of 
five, and a threshold intensity of ten, 883 particles were found in the QD images with a mean intensity of 12.7 and 1624 particles were found in the QR images with a mean intensity of 29.5 . 


\section{References:}

1. Medintz, I.L., Uyeda, H. T., Goldman, E. R., and Mattoussi, H Quantum dot bioconjugates for imaging, labelling and sensing. Nature Materials 4, 435-446 (2005).

2. Alivisatos, A.P., Gu, W. \& Larabell, C. Quantum dots as cellular probes. Ann. Rev. Biomed. Eng. 7, 55-76 (2005).

3. Fu, A., Gu, W., Larabell, C. \& Alivisatos, A.P. Semiconductor nanocrystas for biological imaging. Current Opinion in Neurobiology 15, 568-575 (2005).

4. Michalet, X., Pinaud, F. F., et. al. Quantum Dots for live cells, in vivo imaging, and diagnostics. Science 307, 538-544 (2005).

5. Hu, J.T. et al. Linearly polarized emission from colloidal semiconductor quantum rods. Science 292, 2060-2063 (2001).

6. Manna, L., Milliron, J. D., Meisel, A., Scher E. C., Alivisatos, A. P. Controlled growth of tetrapod-branched inorganic nanocrystals. Nature Materials 2, 382-385 (2003).

7. Milliron, D.J., Hughes, S. M., Cui, Y., Manna, L., Li, J., Wang, L., Alivisatos, A. P. Colloidal nanocrystal heterostructures with linear and branched topology. Nature 430, 190-195 (2005).

8. Ow, H. et al. Bright and Stable Core-Shell Fluorescent Silica Nanoparticles. Nano Lett. 5, 113-117 (2005).

9. Sonnichsen, C., Reinhard, B. M., Liphardt, J., Alivisatos, A. P. A molecular ruler based on plasmon coupling of single gold and silver nanoparticles. Nature Biotechnology 23, 741-745 (2005).

10. J.L. Turner et al. Synthesis of Gadolinium-Labeled Shell-Crosslinked Nanoparticles for Magnetic Resonance Imaging Applications. Advanced Functional Materials 15, 1248-1254 (2005).

11. Tiefenauer, L.X., Kuhne, G. \& Andres, R.Y. Antibody-magnetite nanoparticles: in vitro characterization of a potential tumor-specific contrast agent for magnetic resonance imaging. Bioconjugate Chemistry 4, 347-352 (1993).

12. Nam, J., Thaxton, C. S., Mirkin, C. A. Nanoparticle-based bio-bar codes for the ultrasensitive detection of proteins. Science 301, 1884-1886 (2003). 
13. Manna, L., Scher, E.C., Li, L.S. \& Alivisatos, A.P. Epitaxial growth and photochemical annealing of graded $\mathrm{CdS} / \mathrm{ZnS}$ shells on colloidal CdSe nanorods. Journal Of The American Chemical Society 124, 7136-7145 (2002).

14. Htoon, H., Hollingworth, J. A., Malko, A. V., Dickerson, R., Klimov, V. I. Light amplification in semiconductor nanocrystals: Quantum rods versus quantum dots. Applied Physics Letters 82, 4776-4778 (2003).

15. Shabaev, A., Efros, L. 1D Exciton Spectroscopy of Semiconductor Nanorods. Nano Lett. 4, 1821-1825 (2004).

16. Gerion, D., Pinaud, F., Williams, S. C., Parak, W. J., Zanchet, D., Weiss, S., Alivisatos, A. P. Synthesis and Properties of Biocompatible Water-Soluble SilicaCoated CdSe/ZnS Semiconductor Quantum Dots. J. Phys. Chem. B 105, 88618871 (2001).

17. Parak, W.J. et al. Cell motility and metastatic potential studies based on quantum dot imaging of phagokinetic tracks. Advanced Materials 14, 882-885 (2002).

18. Pellegrino, T. et al. Quantum dot-based cell motility assay. Differentiation 71, 542-548 (2003).

19. Morris, M.C., Depollier, J., Mery, J., Heitz, F. \& Divita, G. A peptide carrier for the delivery of biologically active proteins into mammalian cells. Nature Biotechnology 19, 1173-1176 (2001).

20. Dubertret, B., Skourides, P., Norris, D.J., Noireaux, V., Brivanlou, A. H., Libchaber, A. In Vivo imaging of quantum dots encapsulated in phospholipid micelles. Science 298, 1759-1762 (2002).

21. Voura, E.B., Jaiswal, J. K., Mattoussi, H., Simon, S. M. Tracking metastatic tumor cell extravasation with quantum dot nanocrystals and fluorescence emission-scanning microscopy. Nature Medicine 10, 993-998 (2004).

22. Wu, X., Liu, H., Liu, J., Haley, K. N., Joseph, A. T., Larson, J. P., Ge, N., Peale, F., Bruchez, M. P. Immunofluorescent labeling of cancer marker Her2 and other cellular targets with semiconductor quantum dots. Natuer Biotechnology 21, 4146 (2003).

23. Dahan, M. et al. Diffusion dynamics of glycine receptors revealed by singlequantum dot tracking. Science 302, 442-445 (2003).

24. Lidke, D.S. et al. Quantum dot ligands provide new insights into erbB/HER receptor-mediated signal transduction. Nature Biotechnology 22, 198-203 (2004). 
25. Ebenstein Y., M.T., Banin U. Fluorescence quantum yield of $\mathrm{CdSe} / \mathrm{ZnS}$ nanocrystals investigated by correlated atomic-force and single-particle fluorescence microscopy. Applied Physics Letters 80, 4033-4035 (2002).

26. Giles, R.V., Grzybowski, J., Spiller, D.G. \& Tidd, D.M. Enhanced antisense effects resulting from an improved streptolysin-O protocol for oligodeoxynucleotide delivery into human leukaemia cells. Nucleosides \& Nucleotides 16, 1155-1163 (1997).

27. Itoh, H., Takahashi, A., Adachi, K., Noji, H., Yasuda, R., Yoshida, M., Kinosita K. Jr Mechanically driven ATP synthesis by F1-ATPase. Nature 427, 465-468 (2004).

28. Bruchez, M.J., Maronne, M., Gin, P., Weiss, S., Alivisatos, A. P. Semiconductor nanocrystals as fluorescent biological labels. Science 281, 2013-2016 (1998).

29. Chan, W.C.W., Nie, S. Quantum dot bioconjugates for ultrasensitive nonisotopic detection. Science 281, 2016-2018 (1998).

30. Gao, X., Cui, Y., Levenson, R. M., Chung, L. WK., Nie, S. In Vivo cancer targeting and imaging with semiconductor quanutm dots. Nature Biotechnology 22, 969-976 (2004).

31. Stroh, M., Zimmer, J. P., et. al. Quantum dots spectrally distinguish multiple species within the tumor milieu in vivo. Nature Medicine 11, 678-682 (2005).

32. Kim, S., Lim, YT., Soltesz, EG., De Grand, AM., Lee, J., Nakayama, A., Parker, JA., Mihaljevic, T., Laurence, RG., Dor, DM., et. al. Near-infrared fluorescent type II quantum dots for sentinel lymph node mapping. Nature Biotechnology 22, 93-97 (2004).

33. Medintz, I.L., Clapp, A. R., Mattoussi, H., Goldman, E. R., Fisher, B., Mauro, J. M. Self-assembled nanoscale biosensors based on quantum dot FRET donors. Nature Materials 2, 630-638 (2003). 


\section{Chapter 4}

\section{Discrete Nanostructures of Quantum Dots /Au with DNA}

\subsection{Introduction}

Assemblies of colloidal nanocrystals have received considerable attention in recent years due to their potential for producing functional materials with novel electronic, magnetic and optical properties, which are desirable for applications in biological imaging and detection. ${ }^{1}$ Experiments in which nanocrystals have been induced to form extended aggregates by the pairing of biological macromolecules (DNA, antibodies, etc) have been very successfully exploited in new detection schemes. ${ }^{2} \quad$ If it proves possible to precisely control the number, composition and distance of nanoparticles in a grouping, it may be possible to extend the earlier work, and to create a more powerful set of biological detection schemes. However, it remains a challenge to synthesize discrete nanostructures, especially structures with a greater complexity than dimers and trimers. ${ }^{3}$ In this communication, we demonstrate the synthesis of precise groupings of $\mathrm{CdSe} / \mathrm{ZnS}$ core/shell semiconductor quantum dots (QDs) with $\mathrm{Au}$ nanoparticles. The structures obtained have one QD in the center and a discrete number of $\mathrm{Au}$ nanocrystals (one to seven) attached to it. The nanostructures are generated 
through hybridization of complementary DNA bound to the QD and $\mathrm{Au}$, and subsequent purification using gel-electrophoresis.

QDs are useful biological labels because of their broad excitation spectra and their narrow and size tunable emission spectra, as well as their photo stability. ${ }^{4}$ With improved synthesis of monodisperse nanocrystal samples and bio-conjugation methods to functionalize them, ${ }^{5}$ QDs have begun to be applied in biological experiments and have shown advantages over traditional organic dyes. For example, X. Wu et al. reported multiplexed labeling to distinguish different parts of a single cell by simultaneously exciting different colored QDs; ${ }^{6}$ D. Gerion et al. used them in room-temperature SNP human genotyping and pathogen detection; ${ }^{7}$ and B. Dubertret et al. injected QDs into Xenopus embryos and followed embryonic development up to the late tadpole stage. ${ }^{8}$ In addition to these advances, some recent work has shown that the fluorescence of QDs can be enhanced and blinking of individual dots (random intermittency of the fluorescence) may be reduced by putting QDs in the vicinity of Au surfaces; ${ }^{9}$ this suggests that a structure consisting of a colloidal QD surrounded by Au nanoparticles may possess improved properties over QDs alone. Here, by putting Au nanoparticles around QD using DNA as the scaffolding material, we can control the distance between the Au and the QD as well as the number of Au nanocrystals around the central QD. The complexity and control reported here are considerably higher than in our previous reports of DNA directed nanocrystal assemblies. ${ }^{3}$ Moreover, DNA in the structures is readily manipulated and modified by a large number of enzymes, ${ }^{10}$ which should give them further processibility. Hence, they hold great promise not only as more effective bio-probes but 
also for the fundamental understanding of the physical interactions between QDs and $\mathrm{Au}$ nanoparticles.

\subsection{Synthesis of Discrete Nanostructures of QDs /Au with DNA}

$\mathrm{Au}$ nanocrystals conjugated to one single strand of DNA were prepared and purified using gel-electrophoresis. This technique was developed previously in our group and has been used to group $\mathrm{Au}$ particles into dimers and trimers. ${ }^{3 \mathrm{~b}, 11}$ The use of $\mathrm{Au}$ nanoparticles containing only one DNA excludes cross-linking among particles in subsequent synthesis steps even in very concentrated solutions, thus ensuring high yields for the designed structures. The QD-DNA conjugates were prepared by direct association of biotinylated DNA (Integrated DNA Technologies, Coralville, IA) to streptavidin coated QDots ${ }^{\mathrm{TM}}$ (Quantum Dot Corporation, Hayward, CA). The conjugation is very efficient because of the very high association constant between

streptavidin and biotin. ${ }^{12}$ In a typical synthesis, $26.6 \mathrm{pm}$ biotin-DNA was added to $70 \mu \mathrm{l}$ of $0.19 \mu \mathrm{M}$ colloidal QD solution with a $\mathrm{NaCl}$ concentration of $100 \mathrm{mM}$ and rocked for 3 or 4 hours to form the QD-DNA conjugates. Then, an equimolar amount of Au-1 DNA conjugates with a concentration around $0.1 \mu \mathrm{M}$ (the magnitude varies with each extraction from gel-electrophoresis) were added to the QD-DNA conjugates. Afterwards, the sample was left to rock overnight, allowing for DNA hybridization. Different groupings of nanoparticles were separated using gel-electrophoresis. A typical gel image of the assemblies formed by $10 \mathrm{~nm} \mathrm{Au}, \mathrm{QD}$ 605-streptavidin and 100mer DNA is represented in figure 4.1 . The $1.6 \%$ agarose gel was run in $0.5 \mathrm{x}$ tris-borate-EDTA buffer 
at $6.7 \mathrm{~V} / \mathrm{cm}$ for $1.5 \mathrm{~h}$. The same gel is shown under both UV illumination (left panel) and white light illumination (right panel) to show QD and $\mathrm{Au}$, respectively. Discrete bands are apparent and can be assigned to QD-DNA and Au-1 DNA conjugates, QD with one $\mathrm{Au}\left(\mathrm{QD}(\mathrm{Au})_{1}\right), \mathrm{QD}$ with two $\mathrm{Au}\left(\mathrm{QD}(\mathrm{Au})_{2}\right), \mathrm{QD}$ with three $\mathrm{Au}\left(\mathrm{QD}(\mathrm{Au})_{3}\right)$, and so on. In general, the eye can easily differentiate seven or eight bands, but only bands correlated to

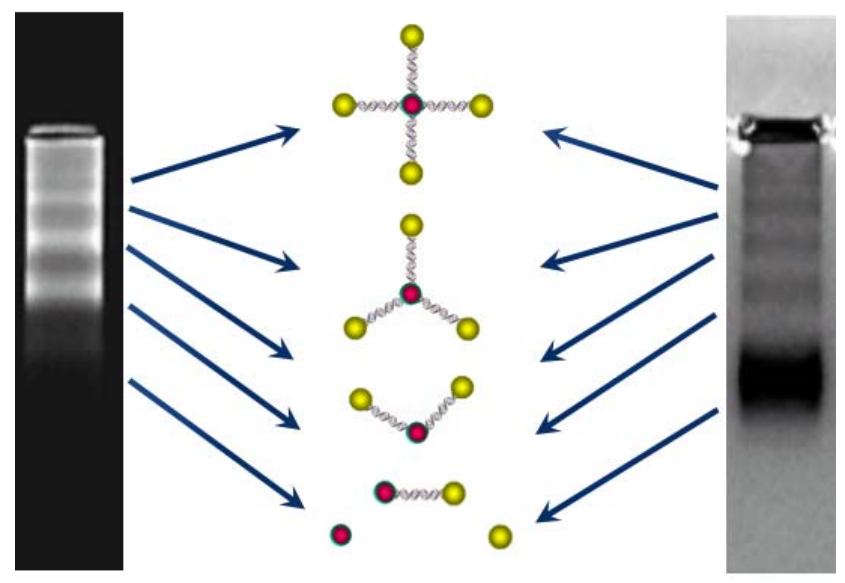

Figure 4.1 Gel-electrophoresis migration patterns of $\mathrm{QD} / \mathrm{Au}$ nanostructures. The same gel is illuminated under UV (to see QDs by fluorescence, left panel) and under white light (to represent Au through absorption, right panel). Discrete bands correspond to different number of $\mathrm{Au}$ (illustrated by cartoon) bound to the QDs through DNA hybridization.

structures with up to $4 \mathrm{Au}$ around the central QD appear in the figure. A more detailed gel electrophoresis image is shown in figure 4.2 that compares the mobility of all related samples. QD-DNA conjugates (lane 5) and Au-1 DNA conjugates (lane 7) in incubation buffer have similar mobilities in the gel. The addition of Au particles around the colloidal QDs increases the size and reduces the mobility so that the assemblies migrate more slowly in a gel (lane 3 and 4, the same sample). The more Au around the central $\mathrm{QD}$, the slower the mobility is. The grouping of QD and Au can only be a result of hybridization of complementary DNA, since neither the mixture of free QD and free Au 
(lane 1), nor the mixture of $\mathrm{QD}$ and Au with non-complementary DNA (lane 2) gives discrete bands in the gel.

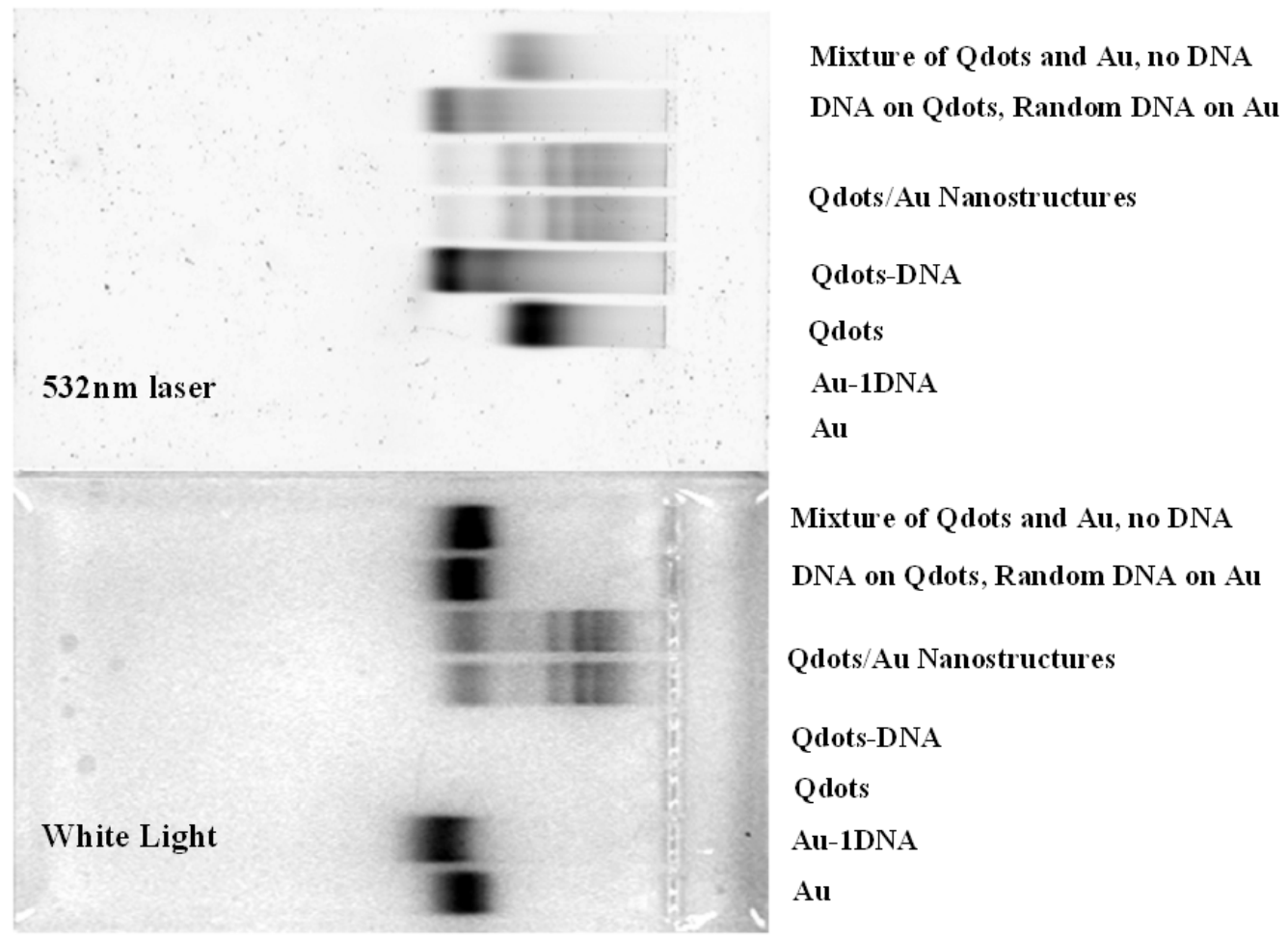

Figure 4.2 Electrophoresis mobility of different samples. The top panel shows QDs. The bottom panel shows Au. Discrete bands only appear when QDs and Au have complimentary DNA on them, which rules out nonspecific binding among QDs, Au and DNA.

\subsection{TEM Analysis of the Nanostructure Populations}

Figure 4.3a-d represents the TEM images of the first four nanostructure bands in the gel. To extract samples from the gel, the band was first cut, and then the gel slice was crushed and left at room temperature overnight in a small amount of TBE buffer. The images show one, two, three, and four Au around the central QD particle, as expected. 
Although the distance between QD and Au in a real structure should be roughly the same since short duplex DNA acts like a rigid rod, in the images they seem to vary because the images are two-dimensional projections of three-dimensional structures. A computer

(a)

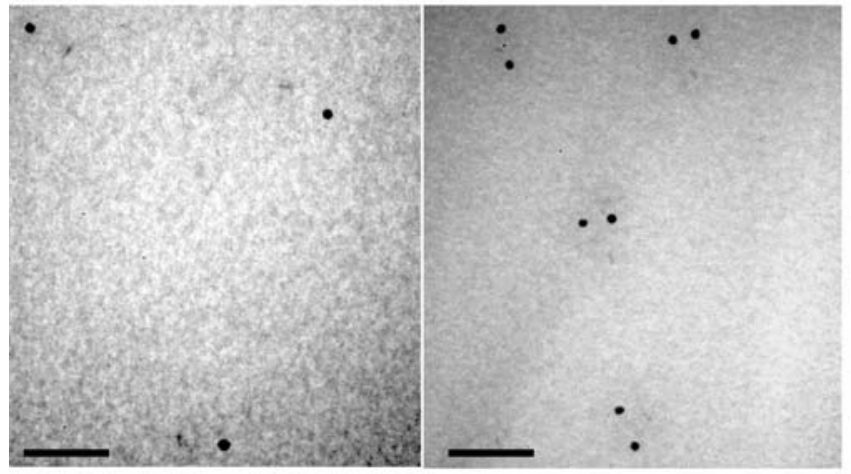

(c)

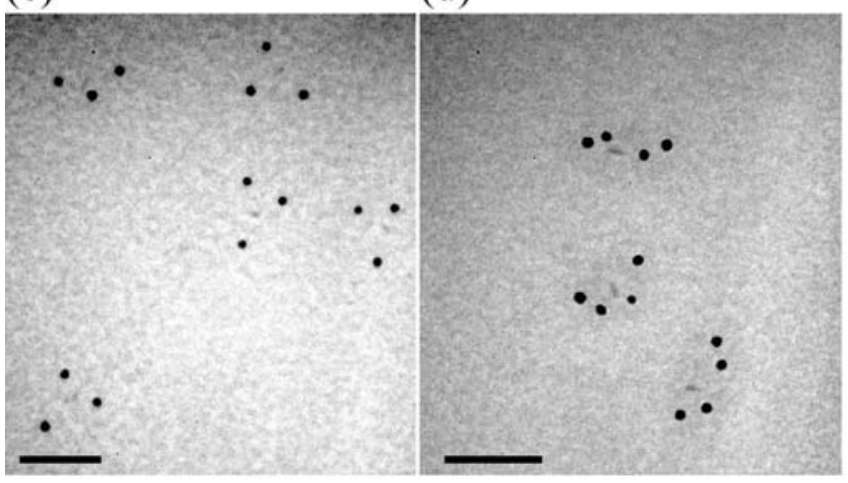

(e)

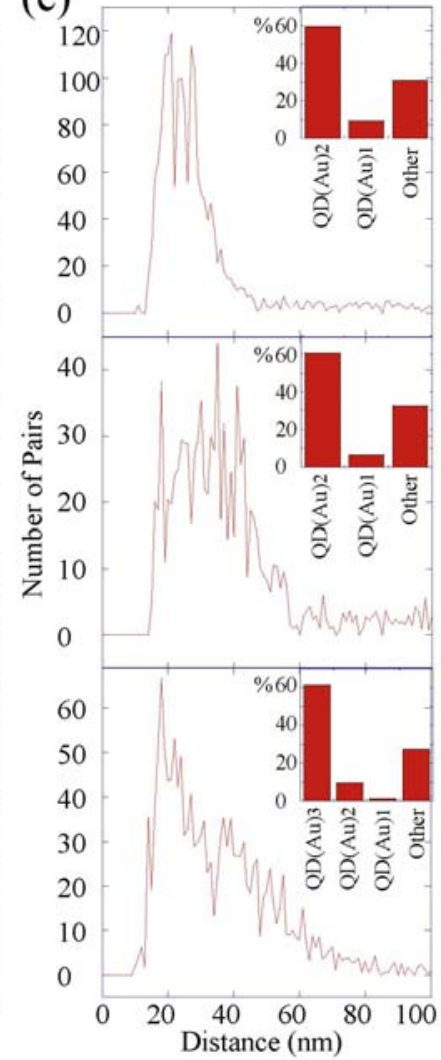

Figure 4.3 TEM images of discrete nanostructures of QDs/Au extracted from corresponding bands after gel-electrophoresis. (a) $\mathrm{QD}(\mathrm{Au})_{1}$. (b) $\mathrm{QD}(\mathrm{Au})_{2}$. (c) $\mathrm{QD}(\mathrm{Au})_{3}$. (d) $\mathrm{QD}(\mathrm{Au})_{4}$. The scale bar is $100 \mathrm{~nm}$. (e) Structure populations and pair distribution functions of $\mathrm{QD}(\mathrm{Au})_{2}$ with 50mer DNA (top), and 100mer DNA connected $\mathrm{QD}(\mathrm{Au})_{2}$ (middle) and $\mathrm{QD}(\mathrm{Au})_{3}$ structures (bottom) based on quantitative analysis of TEM images of corresponding samples.

program was written to extract quantitative data from the TEM images. Figure 4.3e represents the statistical results for nanostructures of $\mathrm{QD}(\mathrm{Au})_{2}$ and $\mathrm{QD}(\mathrm{Au})_{3}$. Since $\mathrm{Au}$ particles have much higher contrast and are clearer in a TEM image than the QDs, the structure populations are calculated based on Au particles in structures vs. the total 
number of Au particles. The statistical analysis on several hundred Au nanoparticles shows that the yields for designed structures are $59.5 \%$ for $\mathrm{QD}(\mathrm{Au})_{2}$ with 50 mer DNA, $60.8 \%$ for $\mathrm{QD}(\mathrm{Au})_{2}$, and $61.5 \%$ for $\mathrm{QD}(\mathrm{Au})_{3}$ with 100 mer $\mathrm{DNA}$, as shown in the insert

of figure 2e. Pair distribution functions showing distances between all QD/Au pairs in each image for these three samples (figure 4.3e) are also consistent with DNA directed assembly. Pair distances reflect the radii of the QDs and Au nanoparticles, the thickness of coating layers, and the length of DNA linkers. Note that the maximum distances observed vary with the length of the DNA connecting the nanoparticles.

\subsection{Conclusions}

In conclusion, discrete nanostructures of QDs surrounded by different numbers of Au have been prepared through hybridization of attached DNA and purified by gelelectrophoresis. Spectroscopic measurements on both ensemble and single molecule scales are subsequently carried out to investigate their optical properties. Rationally designed structures like these open new possibilities for researching novel nanoparticle properties and for developing more efficient nanoprobes. 


\section{References:}

1. (a) Mirkin, C. A.; Letsinger, L. R.; Mucic, C. R.; Storhoff, J. J. Nature 1996, 382, 607. (b) Alivisatos, A. P.; Johnsson K. P.; Peng, X.; Wilson, T. E.; Loweth, C. J.; Bruchex, M. P.; Schultz, P.G. Nature 1996, 382, 609. (c) Josephson, L.; Perez, J. M.; Weissleder, R. Angew. Chem. Int. Ed. 2001, 40, 3204. (d) Nam, J. -M.; Stoeva, S. I.; Mirkin, C. A. J. Am. Chem. Soc. 2004, 126, 5932.

2. (a) Zanchet, D.; Micheel, C. M.; Parak, W. J.; Gerion, D. Williams, C. S.; Alivisatos, A. P. J. Phys. Chem. B 2002, 106, 11758. (b) Loweth, J. C.; Caldwell, W. B.; Peng, X.; Alivisatos, A. P.; Schultz, P. G. Angew. Chem. Int. Ed. 1999, 38, 1808. (d) Perez, J. M.; O’Loughin, T.; Simeone, F. J.; Weissleder, R.; Josephson, L. J. Am. Chem. Soc. 2002, 124, 2856.

3. (a) Alivisatos, A. P. J. Phys. Chem. 1996, 100. 13226. (b) Nirmal, M.; Brus, L. Acc. Chem. Res. 1999, 32, 407. (c) Michalet, X.; Pinaud, F.; Lacoste, T. D.; Dahan, M.; Bruchez, M. P.; Alivisatos, A. P.; Weiss, S. Single Molecules 2001, 2, 261.

4. (a) Murray, C. B.; Norris, D. J.; Bawendi, M. G.; J. Am. Chem. Soc. 1993, 115, 8706. (b) Chan, C. W. W.; Nie, S. Science 1998, 281, 2016. (c) Jaiswal, K. J.; Mattoussi, H.; Mauro, J. M.; Simon, M. S. Nat. Biotechnol. 2003, 21, 47. (e) Parak, W. J.; Gerion, D.; Zanchet, D.; Woerz, A. S.; Pellegrino, T.; Micheel, C. M.; Williams, S. C.; Seitz, M.; Bruehl, R. E.; Bryant, Z.; Bustamante, C.; Bertozzi, C. R.; Alivisatos, A. P. Chem. Mater. 2002, 14, 2113. (f) Pinaud, F.; King, D.; Moore, H. -P.; Weiss, S. J. Am. Chem. Soc. 2004, 126, 6115.

5. Wu, X.; Liu, H.; Liu, J.; Haley, N. K.; Treadway, A. J.; Larson, J. P.; Ge, N.; Peale, F.; Bruchez, P. M. Nat. Biotechnol. 2003, 21, 41.

6. Gerion, D.; Chen, F.; Kannan, B.; Fu, A.; Parak, J. W.; Chen, D.; Majumdar, A.; Alivisatos, A. P. Anal. Chem. 2003, 75, 4766.

7. Dubertret, B.; Skourides, P.; Norris, J. D.; Noireaux, V.; Brivanlou, H. A.; Libchaber, A. Science 2002, 298, 1759.

8. (a) Shimizu, K. T.; Woo, K. W.; Fisher, R. B.; Eisler, J. H.; Bawendi, G. M. PRL 2002, 89, 117401. (b) Kulakovich, O.; Strekal, N.; Yaroshevich, A.; Maskevich, S.; Gaponenko, S.; Nabiev, I.; Woggon, U.; Artemyev, M. Nano lett. 2002, 2, 1450 .

9. (a) Kanaras, G. A.; Wang, Z.; Bates, D. A.; Cosstick, R.; Brust, M. Angew. Chem. Int. Ed. 2003, 42, 191. (b) Pena, R. N. S.; Raina, S.; Goodrich, P. G.; Fedoroff, V. 
N.; Keating, D. C. J. Am. Chem. Soc. 2002, 124, 7314. (c) Yun, C. S.; Khitrov, A. G.; Vergona, E. D.; Reich, O. N.; Strouse, F. G. J. Am. Chem. Soc. 2002, 124, 7644.

10. Zanchet, D.; Micheel, M. C.; Parak, J. W.; Gerion, D.; Alivisatos, A. P. Nano lett. 2001, 1, 32. 


\section{Chapter 5}

\section{Quantum Dots /Au - DNA Nanostructures in Probing the Activity of Hydrolytic Enzymes}

\subsection{Introduction}

The development of techniques to assemble hybrid systems and to monitor their properties is receiving increasing attention because of potential applications of these hybrids in molecular sensing and detection ${ }^{1}$. With increasing capability for manipulating the shape, size, composition and related properties of magnetic, metallic and semiconductor nanocrystals, many new probes incorporating these nanoparticles have been built and have proven useful for a variety of applications. For example, magnetic and metal nanoparticles based bio-barcode have been used for ultrasensitive detection of protein and nucleic acid targets. ${ }^{2,3}$ Positron emission tomography (PET) imaging, magnetic resonance imaging (MRI), optical imaging and other molecular imaging techniques could now be combined in one functional multimodality probe targeting

clinical screening. ${ }^{4,5}$ In most of these hybrid systems, nanoparticles provide useful technology for separation and detection, and also provide means of integrating different 
modalities into one entity due to their advantageous surface area over volume ratio. And properties of each component of the hybrids don't change. However, in some other hybrid systems, nanoparticles alter properties of one or more components within a hybrid system, such as in surface enhanced Raman scattering (SERS) ${ }^{6}$ and in fluorescent resonance energy transfer (FRET) ${ }^{7}$. These systems are intrinsically more complicate but more interesting, because they not only can be used for static imaging, but also have utilities in detecting dynamic molecular interactions.

Closely related to SERS and FRET, the change of fluorescence of chromophores in a hybrid system with metal nanoparticles/surfaces is an interesting subject in recent years. Metal nanoparticles/surfaces have been demonstrated with complex effect for molecules on the surface through basic electromagnetic interactions. Fluorescence quenching is reported when fluorophores are placed near $1.4 \mathrm{~nm} \mathrm{Au}$ nanoparticles. Based on the phenomena, an optical ruler from a hybrid of organic fluorophore and 1.4nm Au particle has been built to measure distance changes up to $25 \mathrm{~nm}^{6}$; A molecular beacon composed of single strand DNA, 1.4nm Au particle and fluorophore has been used for single mismatch detection ${ }^{8}$; And the quenching in complexes of Quantum dots-double strand DNA-1.4nm Au particles is also studied ${ }^{7}$. At the same time, there are published results where fluorescence enhancement is observed when semiconductor nanocrystals are placed in the vicinity of Au surface ${ }^{9,10}$ or when there is superstructures formed between fluorescent semiconductor nanowires and $\mathrm{Au}$ nanoparticles ${ }^{11}$. The complexity within these systems makes intuitive or precise solution to the PL effect of metal upon fluorophores challenging. More experimental results are needed for a clearer 
understanding of the interplay between the enhancement factors and quenching effect. Moreover, as complex structures based on metal and semiconductor nanoparticles are possible to sense temperature changes through the temperature induced length changes of spacing molecules ${ }^{12}$. These structures has opened new horizon for exploring advanced sensing function. Therefore, continuous development of complex structures from fluorophores and metal hybrids is highly desired.

\subsection{Optical Properties of Ensemble QDs/Au-DNA Nanostructures}

We have described in chapter 4 the synthesis of controllable discrete nanostructures from QDs, $\mathrm{Au}$ and DNA ${ }^{13}$, where we can control the sizes of both QDs and Au nanoparticles, adjusting the number of Au nanoparticles around the central QDs, and change the separation between Au and QDs by using DNA with different lengths. Here we report the fluorescence effect of Au upon the photoluminescence of QDs in 50mer, 65mer, 80mer and 100mer DNA connected discrete nanostructures. We see fluorescence quenching in all of the nanostructure samples as extracted after gel-electrophoresis. We utilized this effect to probe the activity of endonuclease EcorI. These nanostructures are very compatible with hydrolytic enzyme functions. Furthermore, we calculated the PL effect in $\mathrm{QDAu}_{1}$ structures, which corresponds well with our experimental data based on ensemble measurements.

Discrete nanostructures of QDs/Au assembled by 50mer, $65 \mathrm{mer}, 80 \mathrm{mer}$, and $100 \mathrm{mer}$

DNA were prepared following similar procedures as reported before ${ }^{13}$. Figure 5.1a 
shows the gel electrophoresis images of 50mer DNA connected quantum dots/Au nanostructures. After gel-electrophoresis, the bands corresponding to $\mathrm{QDAu}_{1}, \mathrm{QDAu}_{2}$, and $\mathrm{QDAu}_{3}$ structures were cut out of the gel and meshed inside a $1.5 \mathrm{ml}$ microcentrifuge tube, followed by addition of $0.5 \mathrm{x}$ TBE buffer and adjusting of $\mathrm{NaCl}$ concentration to $50 \mathrm{mM}$ using $1 \mathrm{M} \mathrm{NaCl}$ solution. The tube was then stored inside $4^{\circ} \mathrm{C}$ refrigerator for three days. This allowed nanostructures to diffuse into the buffer solution. Afterwards, the gel mesh was discarded, and the clear solution was collected into a separate tube and kept at $4^{\circ} \mathrm{C}$. Part of the solution was used for QDs concentration measurement and photoluminescence characterization, and some of the samples were tested in enzyme digestion. A drop (4ul) of the same solution was also dried directly onto the formvar side of a TEM grid (Ted Pella 01890-F) for structural population analysis.

Inductively coupled plasma optical emission spectroscopy (ICP/OES) has a sensitivity of $0.1 \mathrm{ppb}$ for $\mathrm{Cd}^{2+}$ in solution ( $1 \mathrm{ppb}$ is equal to $1 \mu \mathrm{g} / \mathrm{L}$ ), which corresponds to a sensitivity of approximately $0.4 \mathrm{pM}$ of quantum dots. For the nanostructure samples the concentration is on the order of $0.1 \mathrm{nM}$ to $1 \mathrm{nM}$, hundreds of times higher than the detection limit, therefore ICP measurement should provide an accurate measurement on sample concentration. Furthermore, in our experimental range of sample concentrations, the photoluminescence intensity and $\mathrm{Cd}^{2+}$ concentration of free QDs follows a strict linear relationship. Moreover, the photoluminescence intensity is at background level as the $\mathrm{Cd}^{2+}$ concentration goes down to $0 \mathrm{ppb}$. This enables us to use the linear relationship of photoluminescence intensity versus $\mathrm{Cd}^{2+}$ concentration of free QDs to determine the photoluminescence of free QDs at any concentration within our experimental range. 
Hence, we can measure the $\mathrm{Cd}^{2+}$ concentration and photoluminescence of any QDs / $\mathrm{Au}$ nanostructure as extracted from corresponding band after gel-electrophoresis, then compare its photoluminescence intensity with that of free quantum dots at the same concentration. Figure 5.1b shows the photoluminescence ratio of QDs / Au nanostructures over free QDs. Each bar column represents the ratio for a specific 50mer DNA connected quantum dots/Au nanostructue sample as extracted from the corresponding band after gel electrophoresis as shown in Figure 5.1a. PL ratios of roughly $82.6 \%, 74.5 \%$, and $75.2 \%$ are observed in these three samples.
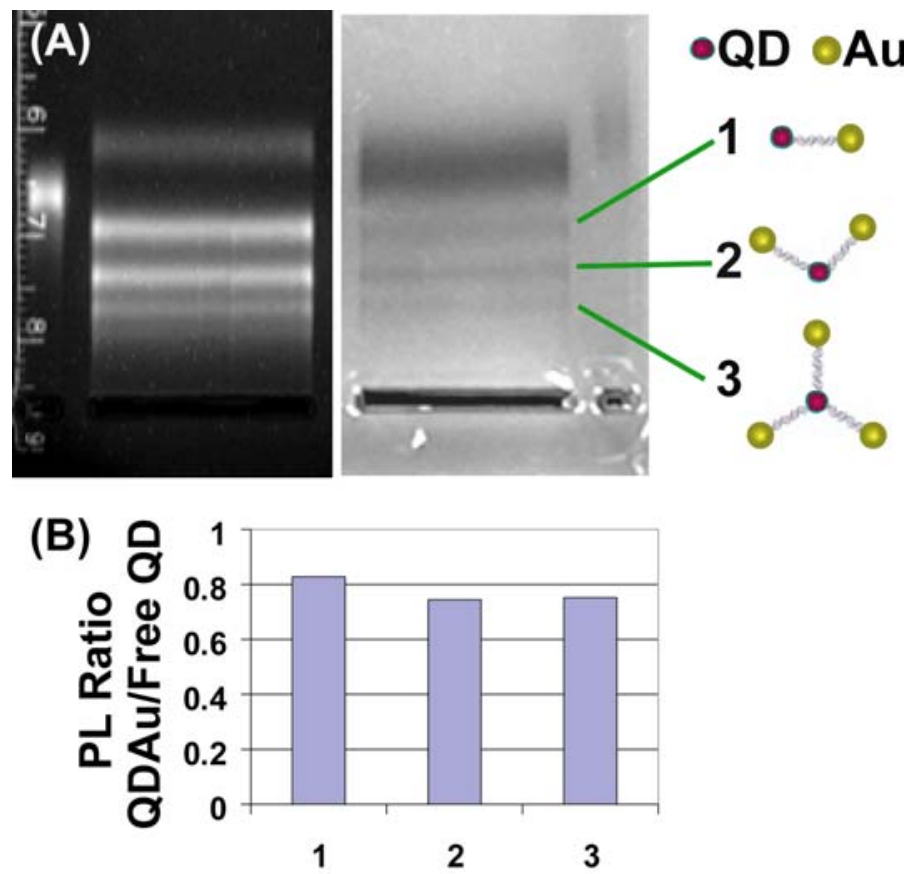

Figure 5.1. (A) Gel-electrophoresis separation of 50mer DNA connected QDAu nanostructures, the left image showing the fluorescence from QD under UV light, and the right image showing the absorption of white light by Au. (B) The photoluminescence of samples extracted from each nanostructures bands are compared with free QD at the same concentration determined by ICP measurements. PL quenching is observed in each sample.

\subsection{QDs/Au-DNA Nanostructures Compatible with Hydrolytic Enzyme Function}


Very importantly, the PL quenching effect can be used to probe hydrolytic enzyme function. Since we use DNA in our experiments as linker molecules between QDs and Au nanoparticles, we can pick up a DNA sequence that contains the recognition sequence of the nuclease interested. Because there is a PL change of QDs in the nanostructures due to nearby Au nanoparticles, there should be a corresponding PL change after the enzymes digest the nanostructure and bring QDs and $\mathrm{Au}$ apart. The same concept should also applicable to peptide connected QDs /Au nanostructures, where the hydrolytic enzyme will be a protease instead, as shown in the cartoon of figure 5.2a. For these hybrid nanostructures with QD and Au nanoparticles linked by a hydrolysable enzyme substrate, there are broad applications for enzyme activity measurements in molecular biology, protein chemistry, and biochemistry. The advantage of these nanostructure-based hybrid sensors is its sensitivity, flexibility in design and assembly, real-time measurement capability, and the extremely small reaction volume it requires for enzyme activity measurement. To prove this concept in principle experimentally, we made the nanostructures with DNA that contains the nucleotide sequence GAATTC at around the middle of the DNA strands. GAATTC is the recognition site for endonuclease EcoRI, a common restriction enzyme. We used the sample extracted from the second nanostructure gel band, because this band extraction showed the biggest quenching effect among the three samples extracted. (Figure 5.1b) It is worth mentioning that some common ingredients in the enzyme storage buffer decrease dramatically the PL of free QDs with time, such as Triton X-100 and 2-Mercaptoethanol. Also, incubation of free QDs in 0.5x TBE at $37^{\circ} \mathrm{C}$ quenches the PL of QDs by $30 \%$ in less than 3 hours. To avoid confusion in data interpretation related to these effects, we checked many different compositions of 
buffer system for EcoRI digestion under room temperature, and found out that a composition of $50 \mathrm{mM} \mathrm{NaCl}, 10 \mathrm{mM} \mathrm{MgCl} 2$ and $0.5 \mathrm{x}$ TBE is the best we can obtain to ensure the stability of QDs and at the same time keep the activity of EcoRI. Hence, EcoRI was dialyzed into a buffer composed of $50 \mathrm{mM} \mathrm{NaCl}, 10 \mathrm{mM} \mathrm{MgCl} 2$ and $0.5 \mathrm{x} \mathrm{TBE}$ ( $\mathrm{pH}$ 8.3). EcorI after dialysis showed similar activity on plasmid DNA cleavage (pREP10 from Invitrogen, Carlsbad, CA) as in original storage buffer, which was demonstrated by the agrose gel electrophoresis in figure 5.2b. For a typical digestion reaction, 2 ul EcoRI, 90ul nanostructure sample, 26.2ul enzyme dialysis buffer and 1.8ul of $0.5 \mathrm{M} \mathrm{MgCl} 2$ are mixed together. PL of the $120 \mathrm{ul}$ mixture was monitored for up to 3

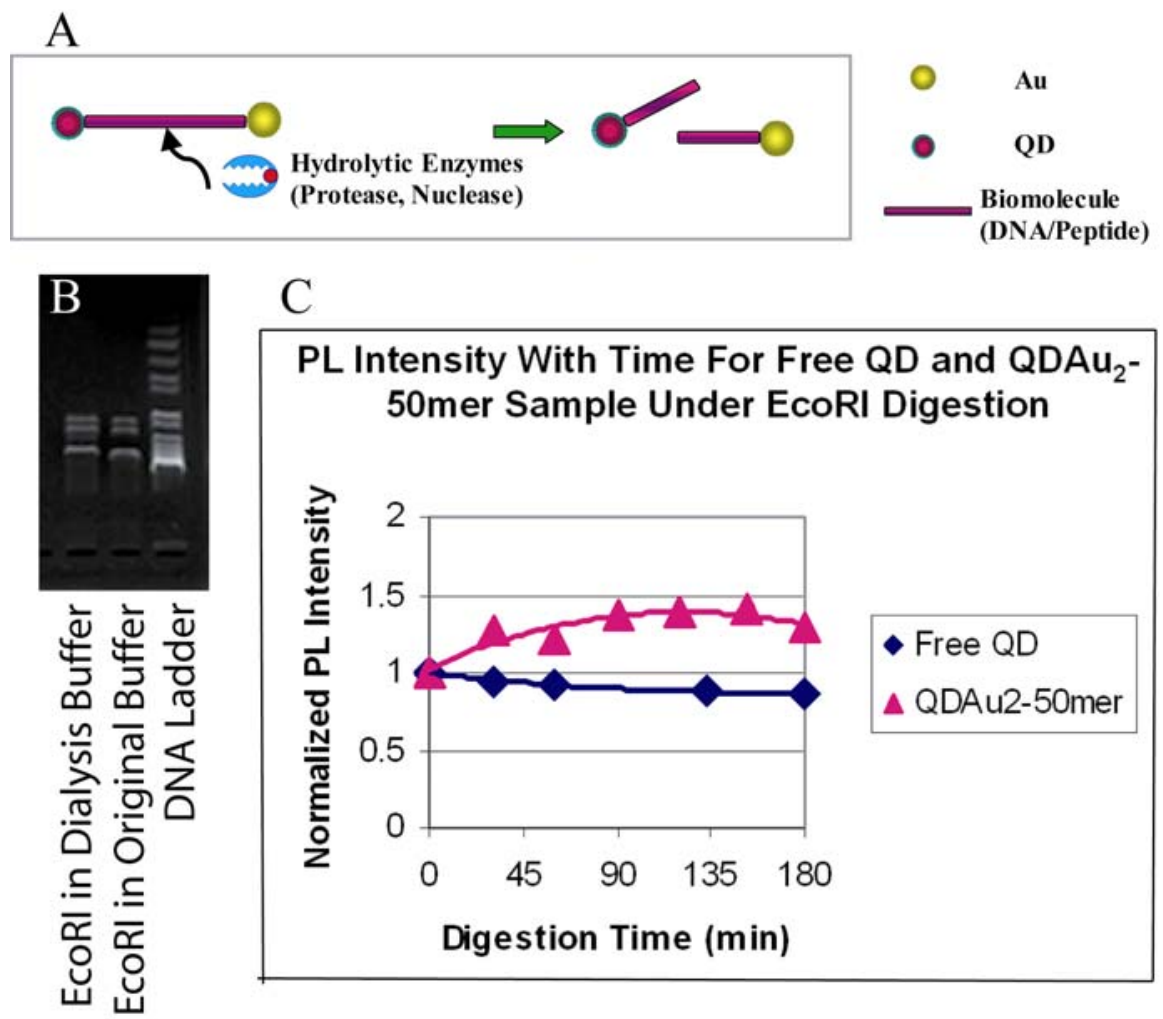

Figure 5.2. (A) A cartoon illustrating the cleavage of the linker biomolecules by hydrolytic enzyme. (B) Gel electrophoresis of the digestion of EcoRI on plasmid DNA pREP10. The left lane shows the digestion in the dialysis buffer of $50 \mathrm{mM} \mathrm{NaCl}, 10 \mathrm{mM} \mathrm{MgCl}$ and $0.5 \mathrm{X} \mathrm{TBE}$; the middle lane shows the digestion in the original EcoRI buffer; the right lane shows the movement of DNA ladder in agarose gel. (C) The effect of Au upon the PL of QD used in probing hydrolytic enzyme function. 
For example, in 50mer DNA connected nanostructure sample extracted from the second structure gel band, the PL of QD is quenched; by choosing double strand DNA containing EcoRI recognition sites, a PL increase can be observed as EcoRI are cleaving the DNA and breaking the structures apart.

hours. A PL increase was clearly seen with time as EcoRI are cleaving the DNA and separating $\mathrm{Au}$ and QDs within a nanostructure. Figure 5.2c shows the digestion curve, where you can see that EcoRI digestion of nanostructures goes fast in the first hour, and is completed around $90 \mathrm{~min}$. This digestion time scale follows well with known digestion time of EcoRI, meaning that the quantum dots/Au nanostructures are very compatible with the digestion procedure. Under the same digestion condition, the PL intensity versus time curve of the control of free QDs mixed with EcorI (blue line, figure 5.2c) is relatively flat, not showing any PL increase as presented for the nanostructure case (red line, figure 5.2c), instead there is a little bit of PL decrease with time, which is because of the remaining instability of QDs under the digestion condition. This does not influence our interpretation and application of quantum dots/Au nanostructure in probing restriction enzyme digestion. However, using QDs with more stable coating should help in the development of nanoprobes based on complex nanostructures and optical properties of quantum dot.

\subsection{Comparison of Photoluminescence Effects between Experimental Results and Analytical Calculation for QDAu1 Structures}

To help understand the quenching effect within the nanostructures and provide more rationale in the design of similar complex systems for biological sensing and detection applications, we have prepared nanostructures connected by different lengths of DNA 
and compared their PL with that of free QD. PL quenching is observed in all of the samples. However, TEM measurement demonstrates that each nanostructure sample as extracted of the agarose gel is not a pure entity, but rather, is composed of a few structural populations. This adds complicity to the interpretation of photoluminescence effects in these nanostructures. Fortunately, we are able to extract three nanostructure samples from the corresponding nanostructure bands for each gel, and do TEM analysis for the structural populations of each sample. Since all samples are composed of a majority of free QD, QDAu1, QDAu2 and QDAu3 structures, (Only a few QDAu structures are seen from nanostructure samples extracted of the third nanostructure band and are ignored in the analysis.) a 3 by 3 matrix was set up to solve the PL effect, which is defined as the ratio of the $\mathrm{PL}$ of $\mathrm{QDAu} \mathrm{n}_{\mathrm{n}}$ - the pure nanostructure type with a specific length of connecting DNA, over free QD. The matrix is set up under the assumption that the photoluminescence of each extracted nanostructure sample is equal to the summation of the photoluminescence contribution from each pure nanostructure type. This assumption is supported by the fact that DNA itself doesn't change the PL of QDs, and there is no inter structural interactions in these nanostructures because the

\begin{tabular}{|l|l|l|l|l|l|l|}
\hline $\begin{array}{l}\mathrm{PL} \\
\text { Effect }\end{array}$ & $\mathrm{QDAu}_{1}$ & $\begin{array}{l}\text { Standard } \\
\text { Deviation }\end{array}$ & $\mathrm{QDAu}_{2}$ & $\begin{array}{l}\text { Standard } \\
\text { Deviation }\end{array}$ & $\mathrm{QDAu}_{3}$ & $\begin{array}{l}\text { Standard } \\
\text { Deviation }\end{array}$ \\
\hline $50 \mathrm{mer}$ & 0.778 & 0.083 & 0.576 & 0.199 & 0.759 & 0.599 \\
\hline $65 \mathrm{mer}$ & 0.944 & 0.116 & 0.431 & 0.092 & 0.420 & 0.178 \\
\hline $80 \mathrm{mer}$ & 0.721 & 0.078 & 0.825 & 0.146 & 1.282 & 0.606 \\
\hline $100 \mathrm{mer}$ & 0.931 & 0.138 & 0.518 & 0.222 & 0.922 & 0.336 \\
\hline
\end{tabular}

Table 5.1. The Au effect upon the PL of QD for each structure type is obtained by solving a matrix built on the assumption that the PL of a sample is equal to the sum of the PL of each structure type in the sample. The standard deviation is obtained through error analysis based on experimental measurements of uncertainty of each component in the matrix. 
photoluminescence of each nanostructure sample goes down linearly with dilution. The solved PL effect for each pure structure type is listed in table 5.1. We will only focus on $\mathrm{QDAu}_{1}$ nanostructures here in the explanation of the interactions of QDs and Au in these nanostructures, as there are bigger experimental uncertainty related to more complicated structures with a higher number of $\mathrm{Au}$ around a central QD, and precise theoretical calculation of the effect in more complicated structures is much more difficult. Nonetheless, the interactions between $\mathrm{QD}$ and $\mathrm{Au}$ in $\mathrm{QDAu}_{1}$ structures should provide insight into the QD and Au interactions in the more complicated structures.

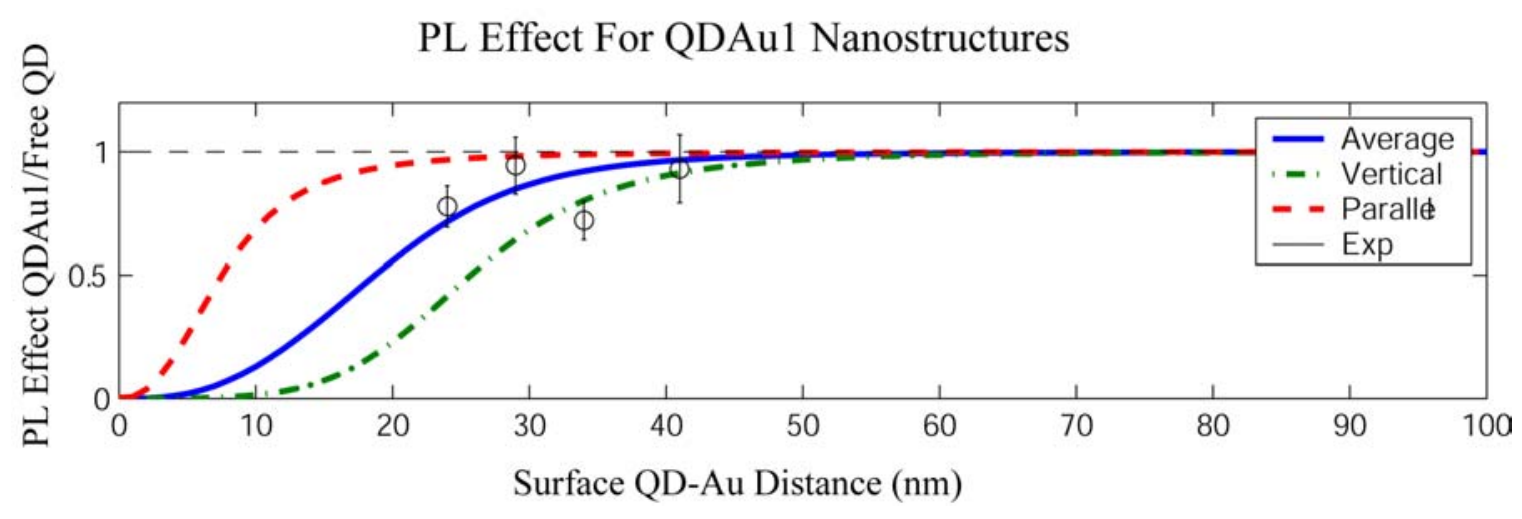

Figure 5.3. Experimental (unconnected points with error bars) and theoretical (colored lines) results of the effect of Au upon the PL of QD for 50mer, 65mer, 80mer and 100mer DNA connected $\mathrm{QDAu} \mathrm{N}_{1}$ Nanostructures.

We build an analytical model to calculate the effect of $\mathrm{Au}$ upon QDs in QDAu nanostructures. In this model, QDs is taken as a simple dipole. The quantum yield of free QDs is defined as:

$$
\mathrm{QY}_{0}=\mathrm{Kr}_{0} /\left(\mathrm{Kr}_{0}+\mathrm{Knr}_{0}\right)
$$

Au plays a role as in the dominating electromagnetic model of surface enhanced Raman and fluorescence scattering ${ }^{10,14}$. To calculate the effect of $\mathrm{Au}$, the incoming, scattering, and internal fields at the excitation frequency are expanded following the well-known 
Mie scattering analysis ${ }^{15-17}$. Then the boundary conditions are applied to obtain the field strength inside and outside the particle. There are two effects from Au. On one hand, it increases the local excitation field by scattering. And the enhanced field may contribute to increased absorption and emission of nearby quantum dots. On the other hand, $\mathrm{Au}$ introduces additional decay channel through plasmon - exciton coupling, where the energy damping is dispersed as Joule heat within Au sphere. The central quantum dot, which is simplified as a point dipole, can be excited and act as an oscillating dipole. Its luminescence is the radiation of the oscillating dipole at the emission frequency. By dividing the emission field into three components, the outgoing radiation (modified radiative decay channel), the nonradiative decay (the same as the free dipole) and internal joule heating (additional non-radiative decay channel), the quantum yield of quantum dots with nearby $\mathrm{Au}$ is written as:

$$
\mathrm{QY}=\mathrm{K}_{\mathrm{r}-\mathrm{Au}} /\left(\mathrm{K}_{\mathrm{r}-\mathrm{Au}}+\mathrm{K}_{\mathrm{nrO}}+\mathrm{K}_{\mathrm{nr}-\mathrm{Joule}}\right)
$$

And the PL effect of Au on QDs in the nanostructures can be expressed as:

$$
\mathrm{PL}_{\text {effect }}=\mathrm{QY} / \mathrm{QY}_{0}{ }^{*} \text { Enhan }
$$

By calculation using values of $\mathrm{QY}_{0}=0.5$, Diameter of $\mathrm{Au}=10 \mathrm{~nm}, \quad \lambda(\mathrm{QD}$ Emission $)=655 \mathrm{~nm}, \lambda($ excitation $)=480$, and $\varepsilon_{\mathrm{H} 2 \mathrm{O}}=1.33$, we obtain the PL effect versus surface distance for the $\mathrm{QDAu}_{1}$ nanostructures as shown in figure 5.3. The green dotted line is the PL effect when the dipole orientation is vertical to the Au surface. The red dashed line is the PL effect when the dipole orientation is parallel to the Au surface. And the average effect over all possible dipole orientations of the solid angle is shown as the blue line. The experimental data for 50mer, $65 \mathrm{mer}, 80 \mathrm{mer}$ and $100 \mathrm{mer}$ DNA connected $\mathrm{QDAu}_{1}$ nanostructures are also plotted in the same figure. The quenching effect of Au to 
quantum dots is clearly manifested in these structures for the center to center distances up to over $50 \mathrm{~nm}$ and levels off at longer distances. The good agreement between the theoretical calculation and the experimental data suggests that electromagnetic interactions are the dominating factor responsible for the effect of Au upon QD in these nanostructures.

\subsection{Discussion}

In previously reported cases where $1.4 \mathrm{~nm} \mathrm{Au}$ is used in discrete complex structures, energy transfer from fluorophores to Au nanoparticles is the exclusive effect, because the small size of $\mathrm{Au}$ makes energy damping a dominating process ${ }^{6-8}$. However, in the discrete nanostructures discussed here, the sizes of $\mathrm{Au}$ are $10 \mathrm{~nm}$, and also are adjustable to bigger sizes, so that not only absorption, but also scattering from Au begin to play a role in the interaction with nearby fluorophores. The competition between energy damping and field enhancement from the same Au result in more intriguing phenomena related to these nanostructures. Although we only clarified the effect in $\mathrm{QDAu}_{1}$ structures with different lengths of DNA here, the extension of observable distance change from $10 \mathrm{~nm}$ of FRET ${ }^{18,19}$ and $25 \mathrm{~nm}$ of SET ${ }^{6}$ to over $50 \mathrm{~nm}$ in these nanostructures is by itself very striking and provide a tool for probing long-range biomolecular interactions not available before. For more complicated nanostructures, though we begin to gain a rough impression on the PL effect, for example, in $\mathrm{QDAu}_{2}$ nanostructures, bigger quenching effects are observed when compared to QDAu nanostructures, more advanced characterization methods need to be developed, including 
single molecule imaging techniques as described in reference $20,{ }^{20}$ to harvest the whole benefits of these assembled QDs / Au nanostructures - for which not only the distances between QDs and $\mathrm{Au}$, but also the sizes of quantum dots and $\mathrm{Au}$, and the number of $\mathrm{Au}$ around a central QDs are all controllable, in probing currently challenging situations of biomolecular interactions over long distance range or with multiple interacting components. 


\section{References:}

1. Katz, E., Willner, I. Integrated Nanoparticle-Biomolecule Hybrid Systems: Synthesis, Properties, and Applications. Angewandte Chemie International Edition 43, 6042-6108 (2004).

2. Nam, J., Thaxton, C. S., Mirkin, C. A. Nanoparticle-based bio-bar codes for the ultrasensitive detection of proteins. Science 301, 1884-1886 (2003).

3. Nam, J.M., Stoeva, S. I., Mirkin, C. A. Bio-Bar-Code-Based DNA Detection with PCR-like Sensitivity. J. Am. Chem. Soc. 126, 5932-5933 (2004).

4. Moseley, M., Donnan, G. Multimodality imaging. Stroke 35, 2632-2634 (2004).

5. Sisk, J. Imaging Cancer One Nanometer at a Time. Radiology Today 4, 12 (2003).

6. Yun, C.S., Javier, A., Jennings, T., Fisher, M., Hira, S., Peterson, S., Hopkins, B., Reich, N. O., Strouse, G. F. Nanometal Surface Energy Transfer in Optical Rulers, Breaking the FRET Barrier. J. Am. Chem. Soc. 127, 3115-3119 (2005).

7. Gueroui, Z., Libchaber, A. Single-Molecule Measurements of Gold-Quenched Quantum Dots. phys. Rev. Lett. 93, 166108 (2005).

8. Dubertret, B., Calame, M., Libchaber, A. Single-mismatch detection using gold quenched fluorescent oligonucleotides. Nature Biotechnology 19, 365-370 (2001).

9. Kulakovich, O., Strekal, N., Yaroshevich, A., Maskevich, S., Gaponenko, S., Nabiev, I., Woggon, U., Artemyev, M. Enhanced Luminescence of CdSe Quantum Dots on Gold Colloids. Nano Lett. 2, 1449-1452 (2002).

10. Shimizu, K.T., Woo, W. K., Fisher, B. R., Eisler, H. J., Bawendi, M. G. SurfaceEnhanced Emission from Single Semiconductor Nanocrystals. phys. Rev. Lett. 89, 117401 (2002).

11. Lee, J., Govorov, A. O., Dulka, J., Kotov, N. A. Bioconjugates of CdTe Nanowires and $\mathrm{Au}$ Nanoparticles: Plasmon-Exciton Interactions, Luminescence Enhancement, and Collective Effects. Nano Lett. 4, 2323-2330 (2004).

12. Lee, J., Govorov, A. O., Kotov, N. A. Nanoparticle Assemblies with Molecular Springs: A Nanoscale Thermometer. Angew. Chem. Int. Ed. 44, 7439-7442 (2005).

13. Fu, A., Micheel, C. M., Cha, J., Chang, H., Yang, H., Alivisatos, A. P. Discrete Nanostructures of Quantum Dots/Au with DNA. J. Am. Chem. Soc. 126, 1083210833 (2004). 
14. Moskovits, M. Surface-enhanced spectroscopy. Review of Modern Physics 57, 783 (1985).

15. Chew, H. Transition rates of atoms near spherical surfaces. Jour. Chem. Phys. 87, 1355 (1987).

16. Kerker, M., Wang, D. -S., Chew, H. Surface enhanced Raman scattering (SERS) by molecules adsorbed at spherical particles. Appl. Opt. 19, 3373 (1980).

17. Ruppin, R. Decay of an excited molecule near a small metal sphere. Jour. Chem. Phys. 76, 1681 (1982).

18. Blanchard, S.C., Kim, H.D., Gonzalez, R. L., Puglisi, J. D., Chu, S. tRNA dynamics on the ribosome during translation. Proc. Natl. Acad. Sci. USA 101, 12893-12898 (2004).

19. Weiss, S. Fluorescence spectroscopy of single biomolecules. Science 283, 16761683 (1999).

20. Zhang, K., Chang, H., Fu, A., Alivisatos, A.P., Haw, Y. Continuous Distribution of Emission States from Single CdSe/ZnS Quantum Dots. Nano Lett. 6, 843-847 (2006). 


\section{Chapter 6}

\section{Conclusion}

Semiconductor nanocrystal QDs, as a new class of biological labels, have been used for many advanced imaging applications due to their superior properties including extreme photostability, multiplexing capability and high brightness. Although they will not totally replace traditional organic fluorophores, as alternative and complimentary probes they are changing the way many biological problems are approached. The same goes for the new nanoprobes developed from semiconductor nanocrystals as described in

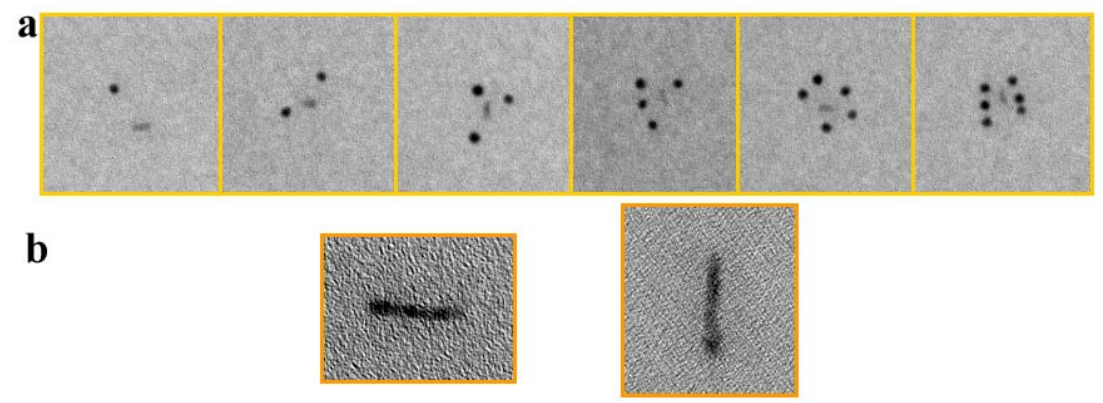

Figure 6.1 New nanoprobes developed in this dissertation research. (a) TEM images of QDAu nanostructures with different numbers of Au connected to the central QD. (b) TEM images of silanized quantum rods.

this dissertation research (Figure 6.1). These new probes, with complimentary or enhanced properties compared to QDs, provide new possibilities for addressing challenging biological imaging and sensing needs. Quantum rods should be better 
fluorescent probes for single molecule imaging as compared to QDs, and could be applied to detect molecular orientations because of their linearly polarized emission. QDAu nanostructures could be used to probe long-range molecular interactions (up to over 50nm) because of the optical effect on QDs from nearby Au nanoparticles. This dissertation research only presents two examples along the road of continuous development of nanoscale science and technology for biomedical applications. As our ability to precisely manipulate materials at the nanometer scale improves, the potential for nanotechnology to enhance human health grows. Safely using nanomaterials for biomedical applications requires further study of their environmental impact and their interactions with living systems. 